A Progress Report and Request for Year 3 Funds Office of Basic Energy Sciences

$$
\begin{aligned}
& \text { from the } \\
& \text { University of Southern California } \\
& \text { Center for Earth Sciences } \\
& \text { Los Angeles, CA } 90089-0740
\end{aligned}
$$

DOE/ER/ 13807--T1

DE92 003394

\title{
CSDP: SEISMOLOGY OF CONTINENTAL THERMAL REGIME
}

Continuation of DOE Contract DE-FG03-87ER 13807

Principal Investigator:

Period of Performance:

Total Cost:

USC Cost Sharing:

Total DOE Cost:

USC Business Office Contact:
Keiiti Aki

W. M. Keck Foundation Professor of Seismology

Sept. 15, 1989 - Sept. 15, 1990

$\$ 183,039$

$\$ 18,652$

$\$ 164,387$

Nann Bennett

Dept. of Contracts and Grants

University of Southern California

Los Angeles, CA 90089-1147

Endorsements:
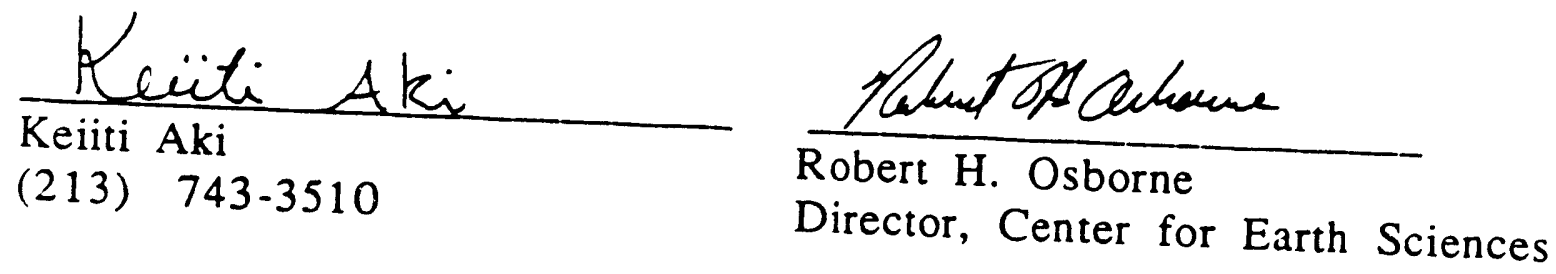

Cornelus J. Pings

Senior Vice-President for Academic Affairs 
TABLE OF CONTENTS

INTRODUCTION-

LIST OF PUBLICATIONS AND PRESENTATIONS IN THE

PAST TWO YEARS

SUMMARY OF RESULTS

PROPOSED RESEARCH FOR THE NEXT YEAR

REFERENCES-.....- 33

RESEARCH BUDGET

\section{CURRICULM VITAE AND CURRENT SUPPORT OF PRINCIPAL INVESTIGATOR}

\section{DISCLAIMER}

wepared as an account of work sponsored by an agency of the United States Neither the United States Government nor any agency thereof, nor any of their Government. Neither the United States Governlied, or assumes any legal liability or responsiemployees, makes any warranty, express or implied, or assum information, apparatus, product, or bility for the accuracy, completeness, or usefulness of any information, appanatus, product Referprocess disclosed, or represents that its use would not inf, or service by trade name, trademark, ence herein to any specific commercial product, process, or se imply its endorsement, recommanufacturer, or otherwise does not necessarily constiture or anply agency thereof. The views mendation, or favoring by the United States Government or any state or reflect those of the

and opinions of authors expressed herein do not 


\section{Progress Report for the Past One Year}

(June 1, 1988 - April 30, 1989)

Introduction

This is a progress report for the past one year of research (year 2 of 5 -year project) under the project titled "CSDP: Seismology of Continental Thermal Regime", in which we proposed to develop seismological interpretation theory and methods applicable to complex structures encountered in continental geothermal areas and apply them to several candidate sites for the Continental Scientific Drilling Project.

During the past year, two Ph.D. thesis works were Jmpleted under the present project. One is a USC thesis on seismic wave propagation in anisotropic media with application to defining fractures in the earth. The other is a MIT thesis on seismic $Q$ and velocity structure for the magma-hydrothermal system of the Valles Caldera, New Mexico. The P.I. co-organized the first International Workshop on Volcanic Seismology at Capri, Italy in October, 1988, and presented the keynote paper on the "state-of-the-art of volcanic seismology". We presented another paper at the workshop on "Assorted Seismic Signals from Kilauea Volcano, Hawaii". Another international meeting, namely, the Chapman Conference on seismic anisotropy in the earth's crust at Berkeley, California in May, 1988, was co-organized by the co-P.I. (P.C.L), and we presented our work on seismic waves in heterogeneous and anisotropic media.

Adding the publications and presentations made in the past year to the list for the preceding year, the following table lists 21 papers published, submitted, or presented in the past two years of the present project.

\section{$\underline{\text { List of Publications and Presentations in the Past Two vears }}$}

1. Koyanagi, R., Chouet, B., and K. Aki, The origin of volcanic tremors, Part I: Data from Hawaiian Volcano Observatory 1969-1985, U.S.G.S. Professional paper 1350, ed. R.W. Decker; T.L. Wright and P.H. Stauffer, $1221-159,1987$. 
2. Chouet, B., R. Koyanagi, and K. Aki, The origin of volcanic tremors, Part II: Theory and discussion, U.S.G.S. Professional Paper 1,50, ed. R. W. Decker, T.L. Wright and P.H. Stauffer, 1259-1280, 1987.

3. Ferrazzini, V. and K. Aki, Slow waves trapped in a fluid-filled infinite crack: Implication for Volcanic Tremor, J. Geophys. Res., 9.2, 92159223, 1987.

4. Leary, P.C., Y.G. Li and K. Aki, Observation and modeling of fault zone fracture seismic anisotropy - I. P, SV and SH travel times, Geophys. J. R. astr. Soc., 91, 461-484, 1987.

5. Li, Y.G., P.C. Leary and K. Aki, Observation and modeling of fault zone fracture seismic anisotropy - II. P-wave polarization anomalies, Geophy. J.R. astr. Soc., 91, 485-492, 1987.

6. K. Aki, Impact of earthquake seismology on the geological community since the Benioff zone, The Centennial Bulletin of the Geological Society of America, 100, 625-629, 1988.

7. Li, Y.G., P.C. Leary and K. Aki, observation and modeling of fault zone fracture seismic anisotropy - III. Trapped Love and Rayleigh Modes, submitted to Bull. Seis. Soc. Am., 1988.

8. Leary, P.C., D.M. Manov and Y.G. Li, A fiber optical borehole seismometer, submitted to Geophys. Res. Letters, 1988.

9. Leary, P.C., Y.G. Li and D.M. Manov, A microprocessor based borehole seismic sonde, submitted to Geophysics, 1987.

10. Ferrazzini, V., K. Aki, B. Chouet, and M. Fehler, Very slow waves trapped in a fluid layer with application to the long period events. Hawaii Symposium on How Volcanoes work, abstract volume, Jan. 1987.

11. Roberts, P.M. and K. Aki, A study of the 2-D Seismic structure of the Valles Caldera, New Mexico using discrete wave-number modeling of observed teleseismic P-waves, EOS, 68, 1347, 1987.

12. Leary, P.C. and Y.G. Li, Observation and modeling of fault-zone trapped Rayleigh modes, Oroville, C A. Annual meeting of Seis. Soc. Am., Honolulu, May, 1988.

13. Ferrazzini, V., K. Aki, B. Chouet and M. Fehler, Hydrofracture experiments at Fenton Hill, New Mexico; Implication for volcanic tremor, Annual meeting of Seis. Soc. Am., Honolulu, May, 1988.

14. Benites, R. and K. Aki, Seismic response of inhomogeneous layered media with irregular interfaces by Gaussian Bean Method, Annual meeting of Seis. Soc. Am., Honolulu, May, 1988.

15. Li, Y. G., P.C. Leary and K. Aki, VSP fracture study of Mohave desert and 3-D modeling by ray tracing, Annual meeting of Seis. Soc. Am., Honolulu, May, 1988. 
16. Ferrazzini, V., B. Chouet, M. Fehler and K. Aki, Quantitative analysis of longperiod events recorded during hydrofracture experiments at Fenton Hill, New Mexico, submitted to J. Geophys. Res., 1989.

17. Ferrazzini, V. and K. Aki, Assorted seismic signals from Kilauea volcano, Hawaii, International Workshop on Volcanic Seismology, Capri, Italy, Oct. 1988.

18. Aki, K., State-of-the-art in Volcanic Seismology, Proc. International Workshop on Volcanic Seismology, Capri, Italy, Oct. 1988, in press.

19. Li, Y.G., P.C. Leary and K. Aki, A ray series approach to travel times and amplitudes in layered, heterogeneous anisotropic media. Third International Workshop on Seismic Anisotropy, Chapman conference on seismic anisotropy in the earth's crust, Berkeley, Calif., 1988.

20. Li, Y.G., Seismic wave propagation in anisotropic media with applications to defining fracture in the earth, Ph.D. thesis, University of Southern California, 262 pp., 1989.

21. Roberts, P.M., Seismic $Q$ and velocity structure for the magma-hydrothermal system of the Valles caldera, New Mexico, Ph.D. thesis, Massachusetts Institute of Technology, 332 pp., 1989. 


\section{Summary of Results}

In the following, we shall summarize results obtained from our works during the past year. We shall start with the paper by the P.I. on the "state-of-the-art in volcanic seismology" because it reviews the current status of seismological methods for studying structures and processes under volcanoes and geothermal sites and tries to point promising directions for future researches. It also serves as an excellent introduction to the main items of the progress report on our project, because it describes the P.I.'s principles guiding the proposed research work.

(1) State-of-the-art in Volcanic Seismology.

The subject of Volcanic Seismology is the most difficult to study among all the subjects seismologists have encountered on the earth. This is because seismic sources in volcanoes involve dynamic motion of gas, fluid and solid, and propagation paths in vulcanoes are usually extremely heterogeneous, anisotropic and absorptive, with irregular topographies and interfaces including cracks in all scales and orientations. Thus, fluid-and gas-filled cracks, heterogeneity, anisotropy and irregular geometry of boundary are the recurring themes of our project as exemplified by the items discussed later in the present progress report.

Our experience indicates that conventional approaches in seismology developed primarily for stratified subsurface structure have not always been useful for volcanic seismology.

For example, we don't find very often convincing cases for a successful reflection survey in a volcanic area, except for some notable cases such as the sedimentary cover acting as a cap for vapor reservoir in Cerro Prieto, Baja California, a deep refiector in the Rio Grande rift, New Mexico (Brown et al, 1980), and possible magma chambers in the oceanic area (Morton and Sleep, 1985). 
Likewise, the stanidard refraction survey which is most effective to delineating vertical structure of the Earth's crust does not seem to have produced results which strikingly reveal the internal structure of a volcano.

The tomographic method using teleseismic P time residuals, on the other hand, seems to have revealed much more insightful picture of the interior of a volcano, probably because the method is specifically designed to determine the lateral variation of seismic velocity at various depths.

The use of teleseismic tomography has become almost a conventional approach in Volcanic Seismology, largely due to the effort of H.M. Iyer (1988) and his colleagues at the U.S. Geological Survey. This approach has contributed significantly in defining the shape and size of a low-velocity body or a high-velocity body (depending on the nature of surrounding rock) which may be related to the magma body.

This approach can be and should be improved by the inversion of full wave form data using the solution of seismic wave scattering in 3-D structure.

Our study of Valles caldera reported in the present progress report is precisely along this line.

Seismic tomography using local earthquake data should give a finer image of subsurface structure because of their shorter wavelengths than teleseismic $P$ waves. A successful example is Thurber's (1984) detailed study of the structure of Kilauea in which the small low-velocity zone of the volcano's summit magma reservoir has been resolved within the otherwise high-velocity core of the volcano. Other examples can be found in a review paper by Thurber and Aki (1987).

A. Hirn and his colleagues at the University of Paris (Nercessian et al, 1984) improved the revolution of tomographic picture by the use of Moho-reflected (PMP) waves generated by explosions which have higher frequencies than teleseismic $P$ waves. The method worked well for a geothermal area in France, but not for the Long Valley caldera in California because of the complexity of PMP waves in the latter area. Thus, the PMP 
tomography has not yet succeeded to become "conventional". We must keep looking for a new "conventional" method which would apply to general geothermal areas.

In the absence of such a method, however, an alternative approach is to design a custom-made experiment tailored to a particular goethermal site. In our experiment with Kilauea Iki (Aki et al, 1978), a partially frozen lava lake in Hawaii, we found that the use of a variety of unconventional methods was essential for determining its seismic properties. For example, the naturally occurring seismic events (caused by thermal stress due to cooling) above the magma reservoir gave the most detailed information on the lateral extent of the reservoir. Furthermore, the dispersion of Love waves played an important role in defining the magma body, because we could not use high-frequency body waves which suffered strong attenuation and scattering in the severely cracked crust, and Rayleigh waves could not exist because of too low velocity due to the presence of flat-layered magma. The SH waves from an earthquake outside Kilauea Iki provided important information on the Swave velocity structure and the viscosity of magma body. Our lesson learned from this experience was that, case by case, we rieed an ingenous design of custom-made experiment tailored to a particular volcano or geothermal site to have an insightful look at its internal structure and working. This is the general guiding principle of the present project. One of the most fascinating subject of study in volcanic seismology is volcanic (harmonic) tremor. Earlier models of the tremor attributed its harmonic character to the propagation-path effect. According to Omer (1950), the shallow crust of Kilauea volcano is nothing but laminae composed of lava flows, ash beds and intrusives. The magma flow exerts a transverse force on one end of the layers and sets these partially free layers into vibration, in a way similar to bending a deck of playing cards clamped at one end. Thus, in Omer's model, the tremor is caused by a quasi-free vibration of solid layers forming the shallow part of the volcano.

Minakami (1974) also considered that the harmonic character of tremor is not due to the effect of a particular source mechanism but primarily due to the propagation path effect. 
According to him, the tremor is a swarm of so called b-type events which show relatively low-frequency wave trains with a spindle-shaped envelope because of extreme shallowness of its focal depths.

A similar idea is shared by many seismologists, who know that seismograms similar to b-type earthquakes are often observed for nonvolcanic sources such as buried explosions or tectonic earthquakes when the wave path is extremely heterogeneous and absorptive, as often found in a volcanic area.

Another school of seismologists, however, considered that the harmonic character of the tremor is due to the source effect, namely, the resonant vibration of a magma body. It is not easy to resolve this fundamental difference when the data are not adequate. For example, if only records at a single station are available for tremors originating from the same source location, it is impossible to separate the source effect from the path effect.

Recently accumulating high-quality data, however, show unequivocal evidence for the source effect responsible for the harmonic character of tremor at least for some cases. For example, the period of deep tremor observed in Hawaii is common to all the stations spread over a large area (Koyanagi et al, 1987) and the period of tremor changes with time during an episode as in the case of an eruption of Sakurajima (Kamo et al, 1977).

We believe that the correct attitude to resolve this problem is to try to separate the source, path and site effects on the tremor spectra and determine the contribution of each effect case by case. We shall describe later in the present progress report about the modeling of observed tremor spectra which can be convincingly attributed to the source effect.

In the present project, we consistently preferred a fluid-filled crack as the seismic source of volcanic tremor for the following reasons.

Ramberg's (1970) experimental study of magma transport using centrifuges showed that while a finger convection (blob) is the preferred mode of mass transport when the viscosity contrast is relatively low between magma and surrounding media, a flow 
through fractures is the preferred mode when the contrast is very high. For basaltic volcanoes, we often observe spectacular fissure eruptions obviously indicating magma transport through cracks.

Even for andesitic volcanoes with a central crator in a coneshaped body, Nakamura (1977) explained the distribution of sites of flank eruptions by hypothesizing that the vent below the central crator may be formed as an intersection of vertical fractures oriented in various directions. Further, sills and dikes are common geologic features of many volcanic regions. These are some of the reasons why we prefer a fluid-filled crack as the seismic model of tremor source.

A fluid-filled crack model was suggested first by Sassa (1935) who found that the observed pattern of motion surrounding a line of crators of Aso volcano during an active period in 1933 could be explained by an opening-closing mode of a crack coincident with the crator alignment. Recent development in modeling tremor sources by a fluid-filled crack have been summarized by Chouet et al (1987), and also discussed by Chouet, and Ferrazzini and Aki (1988). One of their findings form the application of a fluid-filled crack model io observed spectra of tremor is that the narrowness of observed spectral peak requires a sharp impedance contrast between magma and surrounding solid, which is only possible by the presence of bubbles in the magma.

The role of vesiculation or degassing in magnetic dynamics has been emphasized recently by various authors. Eaton et al (1987) made an unusually complete study of magmatic dynamics during the 1959 eruption of Kilauea volcano, combining continuous tilt observation by means of the Press-Ewing seismograph anci accurate log of eruption of lavva into Kilauea Iki lava lake. They observed many times repeated episodes of magma flow into and draining back. from Kilauea Iki were explained in terms of large-scale vesiculation of lava in the conduit effectively pumping lava from the reservoir up into the lake. During early episodes, volcanic tremor was observed in the eruption phase but not in the drainback phase. In later episodes, however, tremor was observed also in the drain-back phase. 
In general, the amplitude of tremor correlated well with the eruption rate, and also with the tilt near the summit closely related to the amount of magma in the summit reservoir.

In addition to this large-scale cycling of magma between the summit reservoir and Kilauea Iki, Eaton et al (1987) observed a periodic change in tilt, called "tilt storm" with period of about 1 hour for several days before the beginning of eruption. This could be an indication of a smaller scale cycling involving degassing and magma flow. It is very interesting to note here that a similar periodic tilt change accompanied by tretnor activity was observed during the recent eruption of Oshima (Fukuyama, personal communication, 1988).

As described by Ferrazzini and Aki (1988), we made a direct visual confirmation of the role of degassing in generating the so called "gas-piston events" named. by Swanson et al. (1979). We found that the gas-piston event associated with degassing to Puu Oo's crator shows a very similar spectral signature as the long-period event and continuous tremor (which is essentially a swarm of long-period events) occurring under the summit. We feel, therefore, that degassing also plays a major role in generating long-period events and tremors.

Thus, degassing may be playing an important role not only in sustaining the tremor by creating a high impedance contrast but also in generating it. A complete study of volcanic tremor requires a new breed of seismologists who can deal with dynamics of gas and fluid coupled to the seismic radiation observable through a seismograph. Non-linearity of magmatic dynamics at a source of volcanic tremor is probably much stronger than that at an earthquake source. 
(2) Seismic $O$ and velocity structure for the magma-hydrothermal system of the Valles Caldera. New Mexico.

The Valles Caldera lies at the intersection of the Jemmtez Lineament and the western edge of the Rio Grande Rift in northern New Mexico. As shown schematically in Fig. 1 the caldera and its immediate surroundings comprise one of the world's largest and most complex volcanic centers, with a long history of eruptive activity extending up to geologically recent times. We present the results of two different forward modeling methods used to determine the local 2-dimensional seistmic structure directly beneath the caldera. In the first method we modeled observed P-wave delays by ray-tracing. In the second method we modeled spectral amplitude variations using the Aki-Larner discrete wavenumber spectral synthesis technique.

We used six portable seismic event recorders to collect teleseismic data at sites placed along a line bisecting the caldera. The azimuth of the array was approximately online to seismically active regions to the northwest and southeast, so that our observations would be for plane waves arriving nearly parallel to the plane used to specify a 2-D vertical cross-section of the caldera directly beneath the array line. This design minimized the effects of 3-D structure in the recorded data. Two separate deployments of the six instruments along the same line yielded 24 high quality teleseismic signals for on-azimuth events recorded at most of the sites. The two array configurations are shown in fig. 2 and fig. 3 in both plan view and vertical cross-section.

Let us first show the evidence for a magma body by a simple and qualitative, but nevertheless convincing, method using only the measured arrival time of the first $P$ waves. Fig. 4 shows ray paths classified into five categories of delay relative to that expected for a standard laterally homogeneous earth model. This diagram is similar to the one used by Steeples and Iyer (1976) for delineating a low velocity zone (LVZ) beneath the Long Valley Caldera in California. We clearly recognize to existence of $L V Z$ also under the Valles 
caldera. In order to define the LVZ more quantitatively, we made an extensive ray-tracing study for a variety of LVZ models and compared with the observed arrival times.

Fig. 5 shows the best model of LVZ and ray paths corresponding to teleseismic $P$ waves from northwest (upper diagram) and southeast (lower diagram). The comparisons of time delays between observed and predicted using the above best model are shown in Fig. 6 for an event from northwest and Fig. 7 for ap events from southeast. The variance reduction by our best model relative to the laterally homogeneous standard earth model is very high (mosily greater than 90\%) as shown in Table I for 21 events.

The modeling of observed spectral amplitude variations using the Aki, Lamer (1970) method, on the other hand, revealed a totally different aspect of the subsurface stricture. After carefully studying the issonance, interference and scattering effects by topography, caldera fill and magma body, we found that the single most important factor controlling the observed spectral amplitude variation is the absorption by a verry low $\mathrm{Q}$ zone (LQZ) under the caldera.

The best model is shown in Fig. 8, where we find a body of extremely low $Q$ (about 1) beneath the caldera at the depth interval from about $1 \mathrm{~km}$ to $4 \mathrm{~km}$ from the surface. The comparison of the spectral amplitudes between observed and predicted using the best model are shown in Fig. 9 for a southeast event and in fig. 10 for a northwest event. The variance reduction is again very high for both cases.

From the ray-tracing study, we demonstrated that the teleseismic $P$-wave delays could be explained with a model that contains two relatively simple, homogeneous components. These are an irregularly shaped, low velocity caldera fill surface layer and a separate lens-shaped, low velocity inclusion at approximately 10 to $13 \mathrm{~km}$ true average depth beneath the resurgent dome. Although we could not constrain the depths to the top and bottom of the inclusion, we demonstrated that its minimum height is approximately 8 $\mathrm{km}$ and its width at the tapered section is approximately $17 . \mathrm{km}$. Our solution for this 
inclusion was obtained only after fitting as much of the data as possible with the caldera fill layer alone.

From the spectral amplitude study, on the other hand, we found that the one major feature required to explain the low amplitudes inside the caldera was a large zone of very low $Q$ at the bottom of the caldera fill layer. This LQZ was constrained reasonably well to be about $16 \mathrm{~km}$ in width, $4 \mathrm{~km}$ in height and approximately $1 \mathrm{~km}$ deep to its top. To obtain the best fit to the data, we found that a relatively non-attenuating upper caldera fill layer was also required, but that the effects of this feature were secondary to the dominant LQZ effect. It was also necessary that the $\mathrm{LQZ}$ have significantly lower $\mathrm{P}$ and $\mathrm{S}$ velocities than its surroundings, in order to produce strong enough-attenuation. The velocity structure thus preferred for explaining the spectral amplitude data is somewhat inconsistent with the velocity structure obtained from the ray-tracing study.

The above inconsistency may be reconciled if we increase the thickness of caldera fill uniformly in the study area. This increase would deepen the low velocity region to match the low $Q$ zone without significant effect on the observed travel time variation across the area. Fig. 11 shows such a compromised image of subsurface structure where the amplitude model and the ray-racing model are superimposed.

We propose that the above seismic image of the Valles caldera is consistent with its known volcanic evolution. The major stages in the formation of the Valles Caldera were outlined in Smith \& Bailey (1966) as follows. 1) Doming and swelling on a regional scale of the pre-existing Jemez volcanic field and local formation of ring-fractures above the Valles magma chamber. 2) Eruption of the second member of the Bandelier tuff through the established ring-fracture system. 3) Caldera formation due to collapse of the roof the Valles magma chamber and subsidence of the central caldera block along the ring fractures. 4) Caldera lake formation and eruption of the early rhyolites, during which approximately $600 \mathrm{~m}$ of caldera fill accumulated on top of the subsided central block. 5) Resurgence and doming of the central caldera block due to renewed magma rise, during which time the 
middle period rhyolites were erupted from the northwestern ring fracture zone and from the longitudinal graben at the center of the dome. 6) Eruption of the late period rhyolites as isolated domes in the moat area between the resurgent dome and the topographic caldera rim. 7) Hot spring activity in the westem part of the caldera and erosion of the caldera fill. These seven basic stages were recognized by Smith \& Bailey (1966) as being common to the development of all Valles-like resurgent calderas that they examined. The Valles Caldera is currently in the final stage above, which terminates the cycle. The most recent volcanic activity in the caldera occurred about 100,000 years ago (Heiken \& Goff, 1983).

We propose that our modeling results are consistent with the general structural features one might expect to have been formed by the processes governing stages 3 .) through 7.) above. Specifically, our low $Q$ zone is consistent with a highly fractured, partially water-saturated volume of material which could have been formed by the process of post-subsidence magma rise and caldera floor resurgence, followed by development of a large hydrothermal system. Our low velocity zone may be the remains of silicic magma chamber responsible for at least the two major caldera forming eruptions of 1.1 and 1.4 Ma. In fact, the volume of LVZ estimated from our result (ranging from 270 to $630 \mathrm{~km}^{3}$ ) fall within the range of calculation by Kolstad and McGetchin (1978) for three different initial sizes of pluton yielded after cooling for 1 million years. 


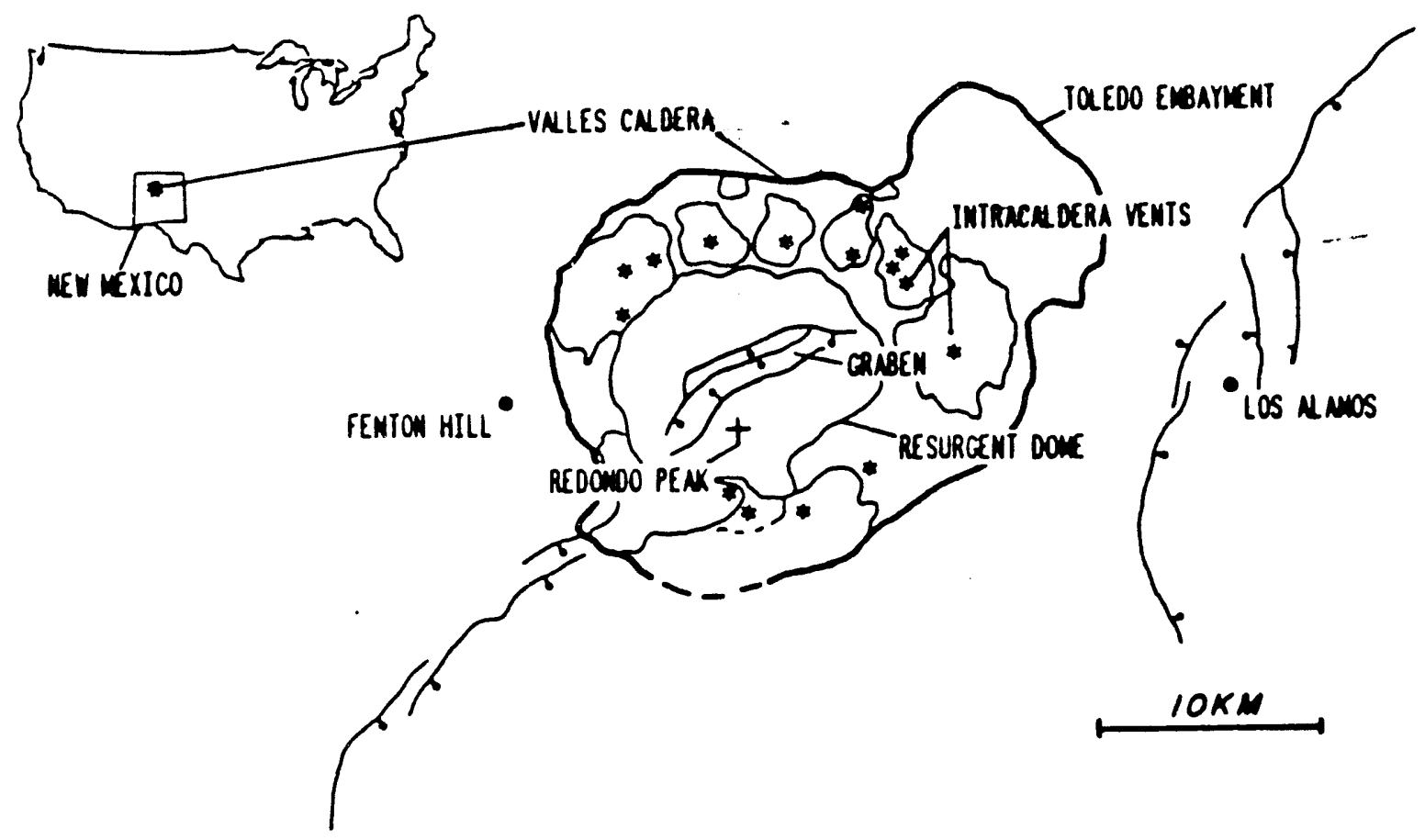

Figure 1.2 Schematic map showing location and major stuctural features of the Valles Caldera. The Jemez Fault Zone is shown to the southwest of the caldera, and the Pajarito Fault Zone is to the east, passing through Los Alamos. The Toledo Caldera is approximately coincident with the Valles Caldera. This figure is reproduced from Felch [1987]. 


\section{SCHEMATIC MAP OF VAUES CALDERA AND STTE LOCATIONS FOR 1987 ARRAY 1}
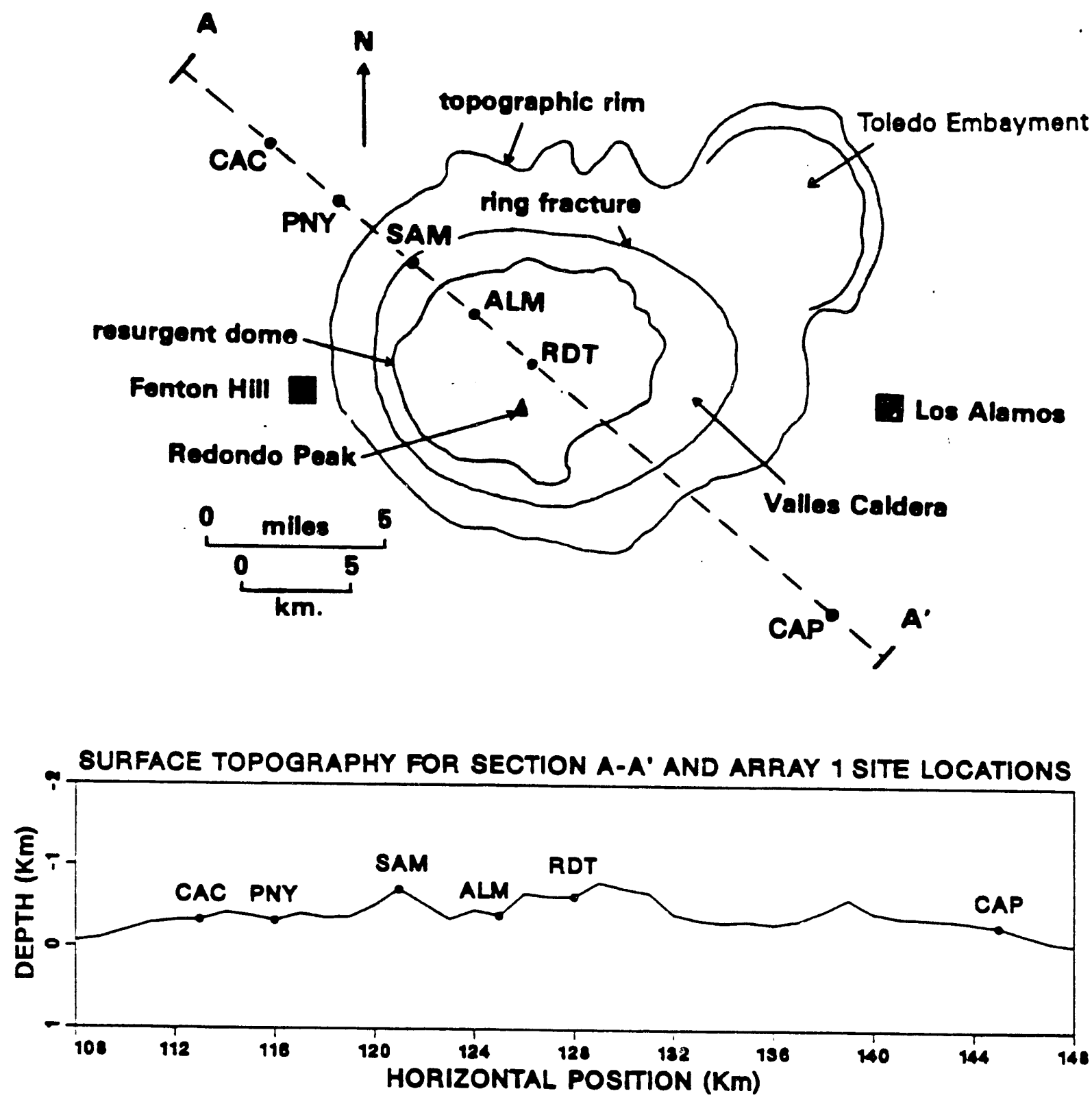

Figure 2.1a Schematic map (top), modified from Nielson \& Hulen [1984], of Jemez Mountains study region in northern New Mexico showing major topographic and structural surface features of the Valles Caldera and seismometer deployment sites used in 1987 for array 1. Vertical crosssectional view of digitized surface topography is shown at the bottom with the six sites indicated. 


\section{SCHEMATIC MAP OF VALLS CALDERA AND SITE LOCATIONS FOR 1987 ARRAY 2}
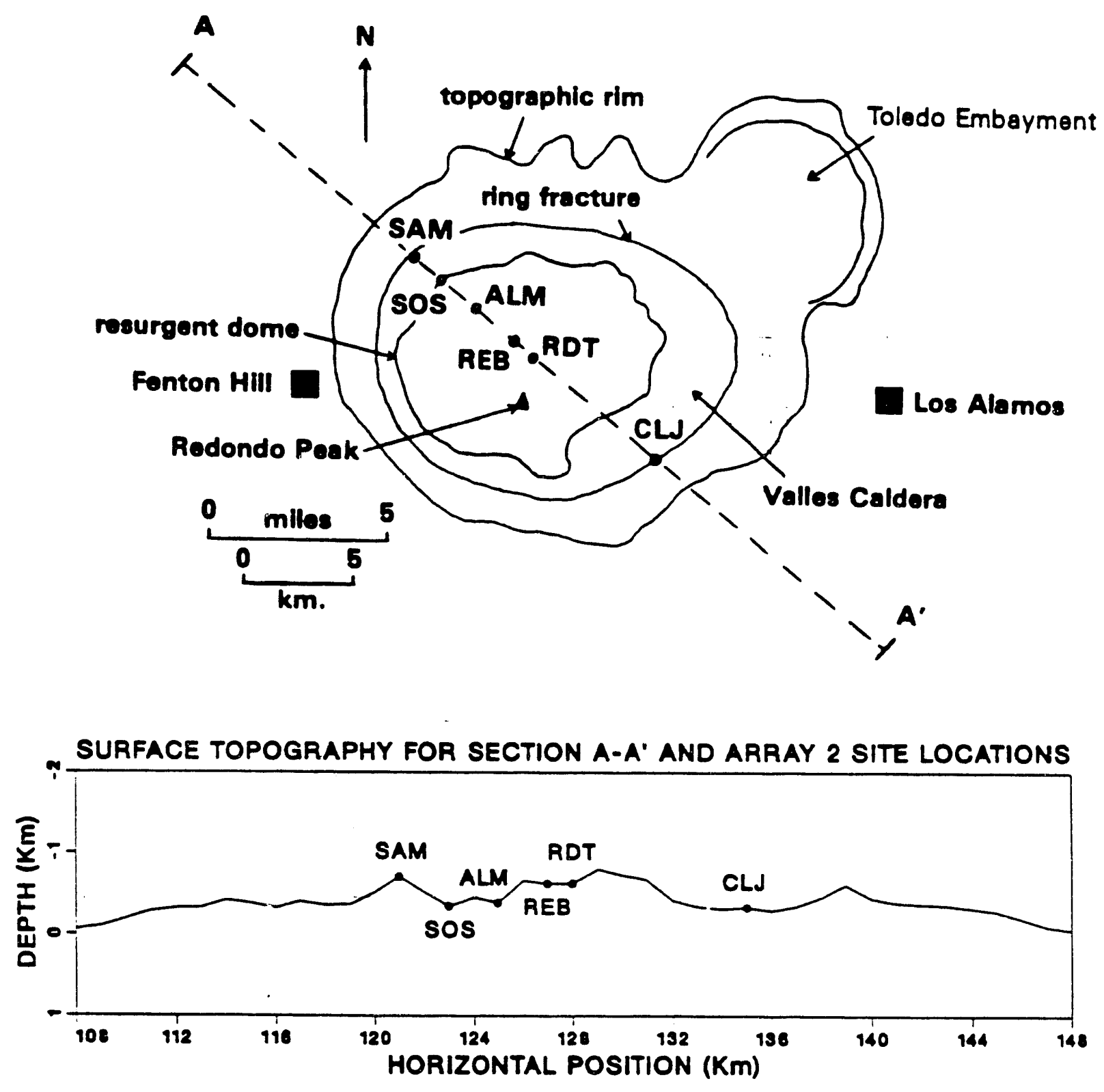

Figure 2.1b Same as Figure 2.1a except site locations shown are for array 2. 


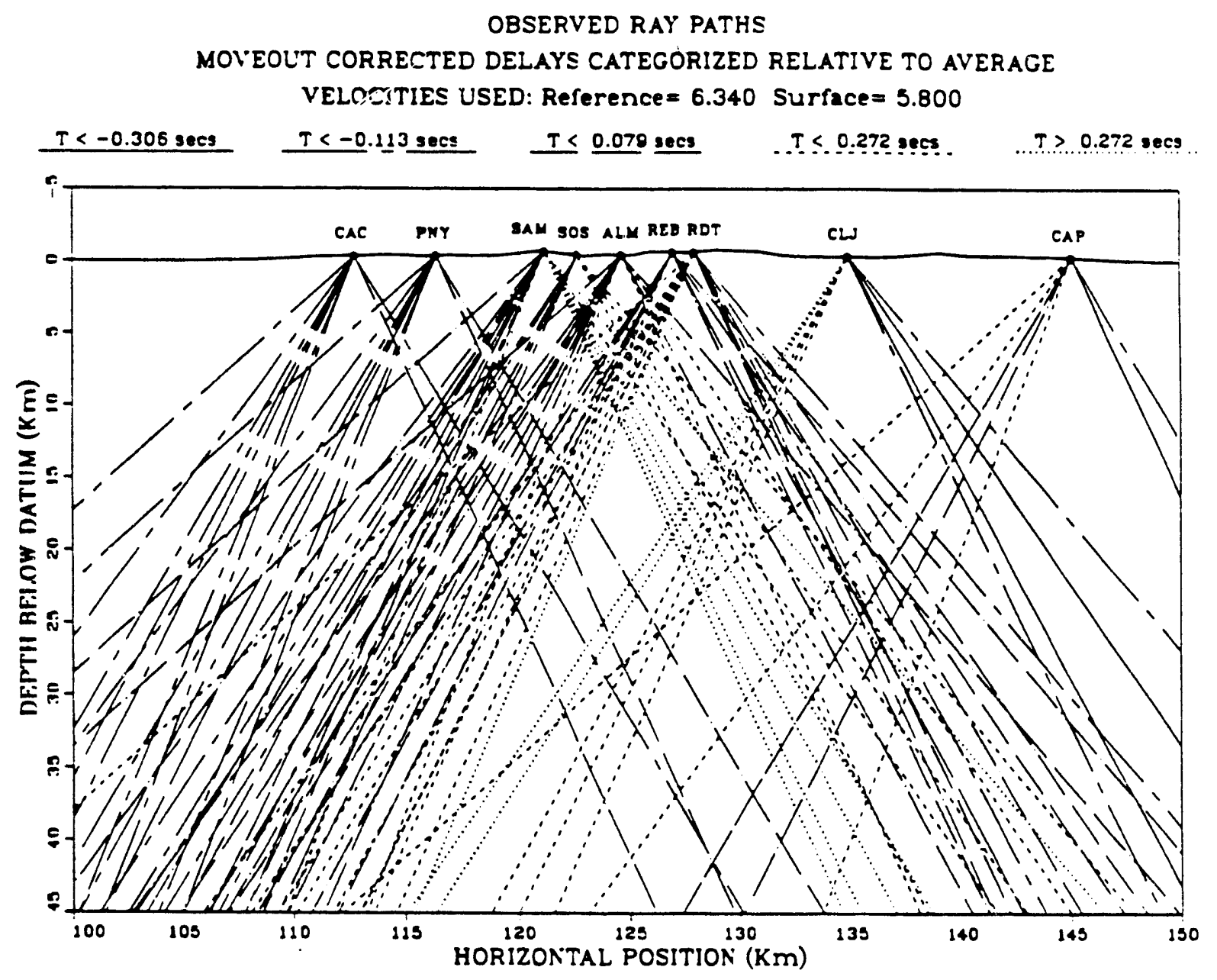

Figure 2.35 Observed ray paths for all events recorded on both arrays 1 and 2. Incidence angles were estimated from Richter [1958] and corrected for the lower near-surface P-wave velocity for the Valles Caldera region. Rays are categorized according to the strengih of the arrival time delay for each one and plotted in different line styles indicated in the legend. Site codes are listed near their locations along the free surface topography profile where the rays end at the top of the figure. 

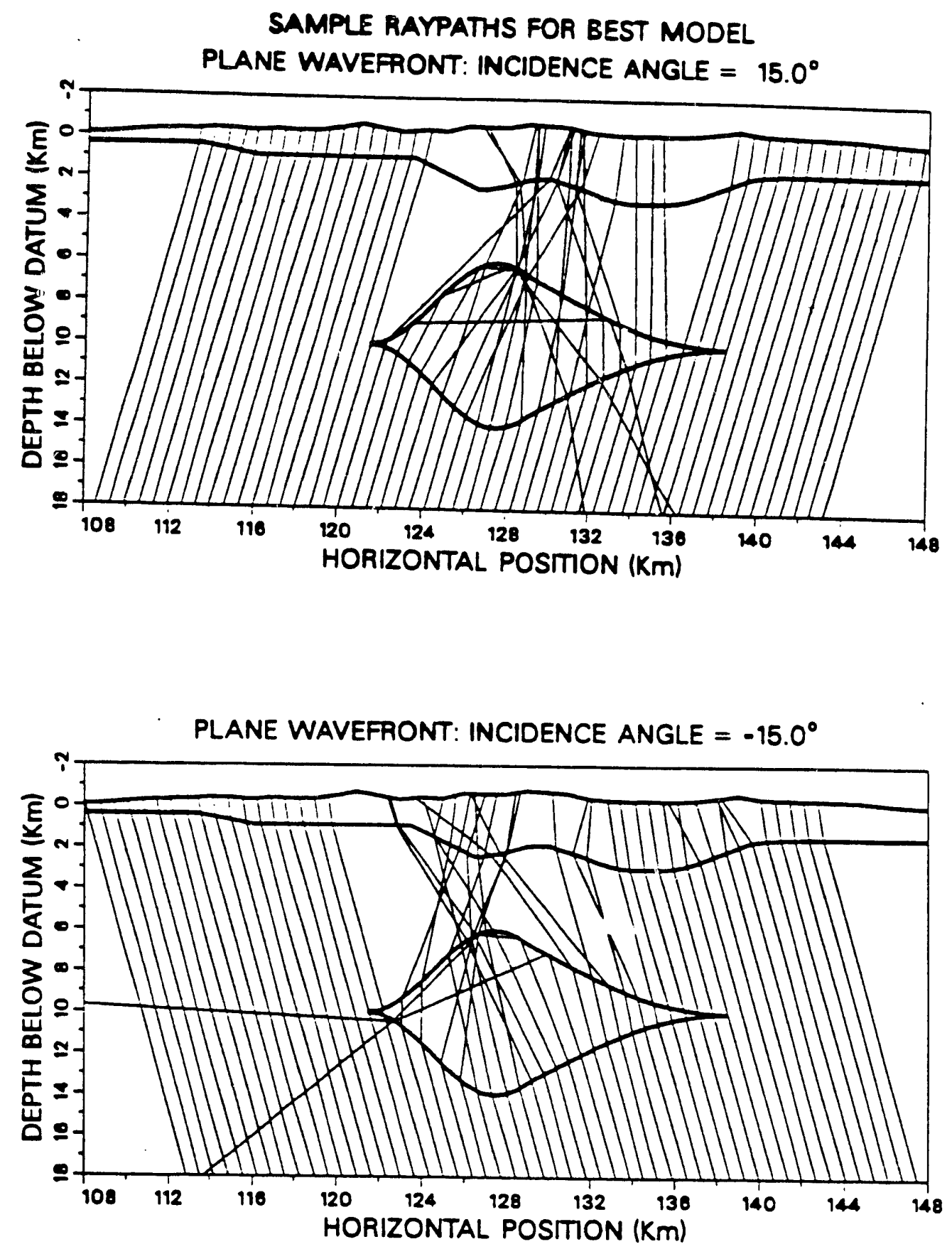

Figure 4.3 Typical raypaths for the best model shown in Figure 4.1 for positive incidence angle $\theta_{0}=+15^{\circ}$ (top) and negative incidence, $\theta_{0}=-15^{\circ}$ (bottom). 


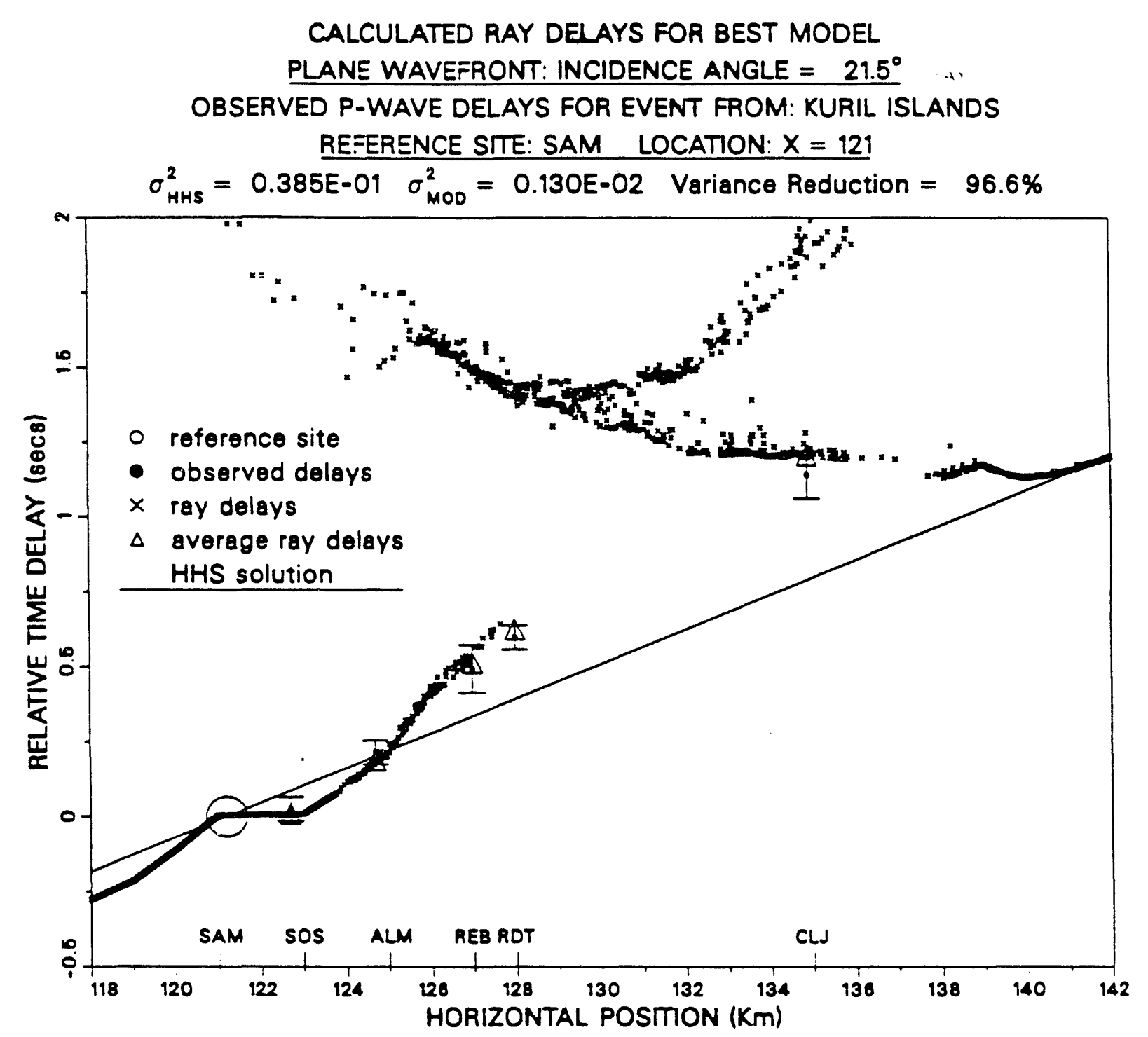

Figure 4.4a Comparison of ray tracing results for the best model, Figure 4.2, with observed delays for the Kuril Islands event shown previously in Figure 4.1b. 
CALCULATED RAY DELAYS FOR BEST MODEL

PLANE WAVEFRONT: INCIDENCE ANGLE $=21.0^{\circ}$

OBSERVED P-WAVE DELAYS FOR EVENT FROM: JU.'UY, ARGENTINA

REFERENCE SITE: CLJ LOCATION: $X:=135$

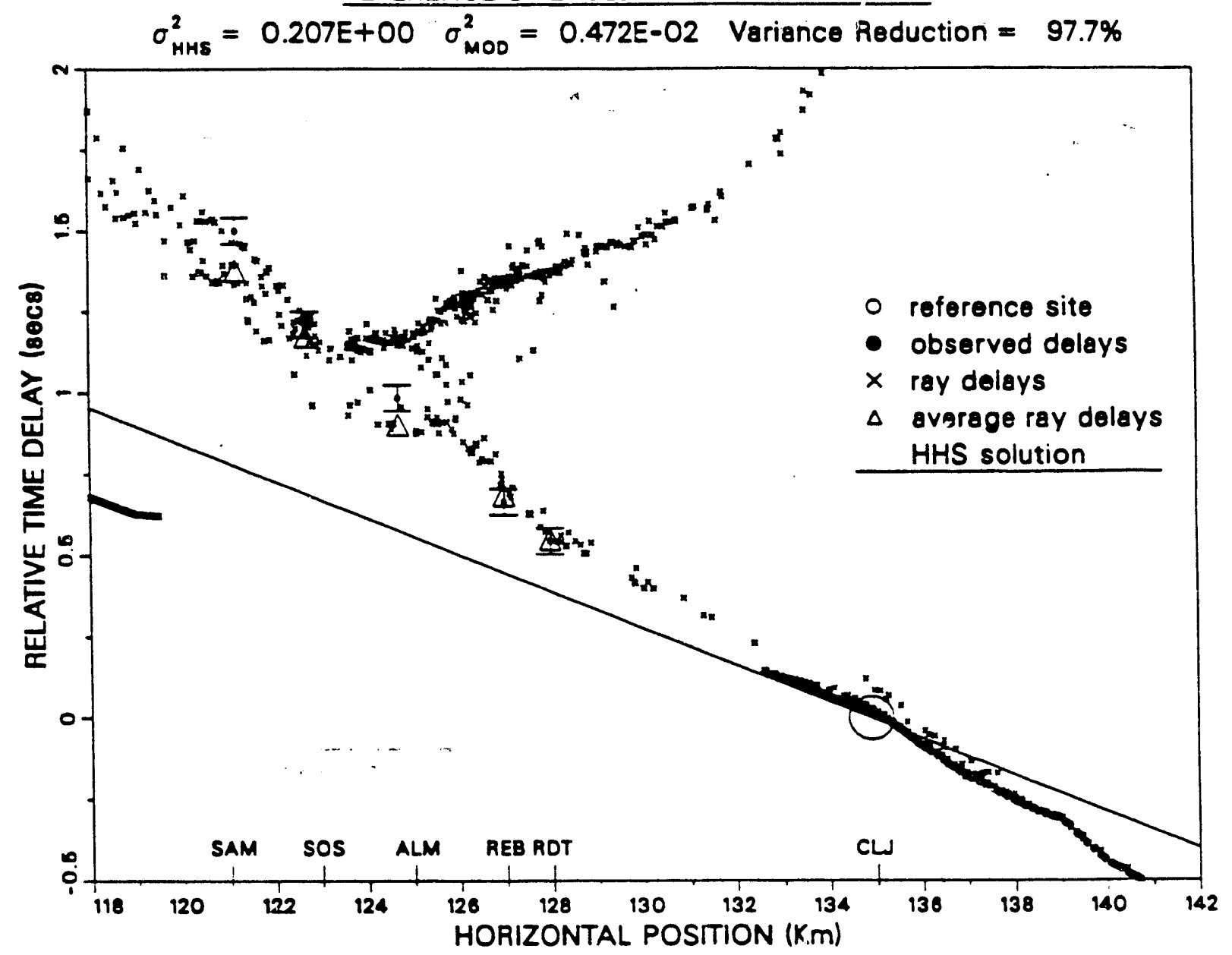

Figure 4.4b Comparison of ray tracing results for the best model with observed delays for the Jujuy event shown previously in Figure 4.1c. 
Table 1

TABLE 4.1

Residual Variance and Variance Reduction for

Best Ray Model Compared with 21 Events

\begin{tabular}{|l|c|c|c|c|c|}
\hline \multicolumn{1}{|c|}{$\begin{array}{c}\text { Event } \\
\text { Region }\end{array}$} & $\theta_{0}$ & $\begin{array}{c}\# \text { of } \\
\text { Points }\end{array}$ & $\sigma_{H H S}^{2}$ & $\sigma_{M O D}^{2}$ & $\begin{array}{c}\text { Variance } \\
\text { Reduction }\end{array}$ \\
\hline Guatemala & -32.0 & 5 & 0.0776 & 0.0065 & $+91.7 \%$ \\
\hline Ecuador & -26.5 & 5 & 0.2010 & 0.0571 & $+71.6 \%$ \\
\hline Southern Peru & -23.0 & 2 & 0.2090 & 0.0649 & $+68.9 \%$ \\
\hline Northern Chile & -21.0 & 4 & 0.2180 & 0.0046 & $+97.9 \%$ \\
\hline Jujuy, Argentina & -21.0 & 5 & 0.2070 & 0.0047 & $+97.7 \%$ \\
\hline Chile-Argentina & -19.0 & 3 & 0.1820 & 0.0049 & $+97.3 \%$ \\
\hline Central Chile & -18.0 & 3 & 0.5230 & 0.0023 & $+99.6 \%$ \\
\hline South of Honshu & +15.0 & 3 & 0.1160 & 0.0360 & $+68.9 \%$ \\
\hline Coast of Honshu & +17.0 & 3 & 0.2050 & 0.0218 & $+89.4 \%$ \\
\hline Coast of Honshu & +18.0 & 5 & 0.0449 & 0.0044 & $+90.2 \%$ \\
\hline Kuril Islands & +20.0 & 4 & 0.1450 & 0.0145 & $+90.0 \%$ \\
\hline Sea of Okhotsk & +20.0 & 3 & 0.0762 & 0.0149 & $+80.4 \%$ \\
\hline Kuril Islands & +20.0 & 5 & 0.0282 & 0.0049 & $+82.5 \%$ \\
\hline Kuril Islands & +21.5 & 4 & 0.1070 & 0.0077 & $+92.8 \%$ \\
\hline Kuril Islands & +21.5 & 5 & 0.0385 & 0.0013 & $+96.6 \%$ \\
\hline Kommandorsky I. & +23.5 & 4 & 0.0833 & 0.0027 & $+96.6 \%$ \\
\hline Kommandorsky I. & +23.5 & 4 & 0.0894 & 0.0053 & $+94.1 \%$ \\
\hline Andreanof Islands & +24.5 & 5 & 0.0586 & 0.0002 & $+99.7 \%$ \\
\hline Fox Islands & +26.0 & 5 & 0.0466 & 0.0012 & $+97.4 \%$ \\
\hline Kodiak Island & +28.5 & 4 & 0.0595 & 0.0021 & $+96.4 \%$ \\
\hline Coast of Oregon & +40.0 & 4 & 0.0631 & 0.0011 & $+98.2 \%$ \\
\hline TOTAL & - & 95 & 0.1080 & 0.0097 & $+91.0 \%$ \\
\hline
\end{tabular}




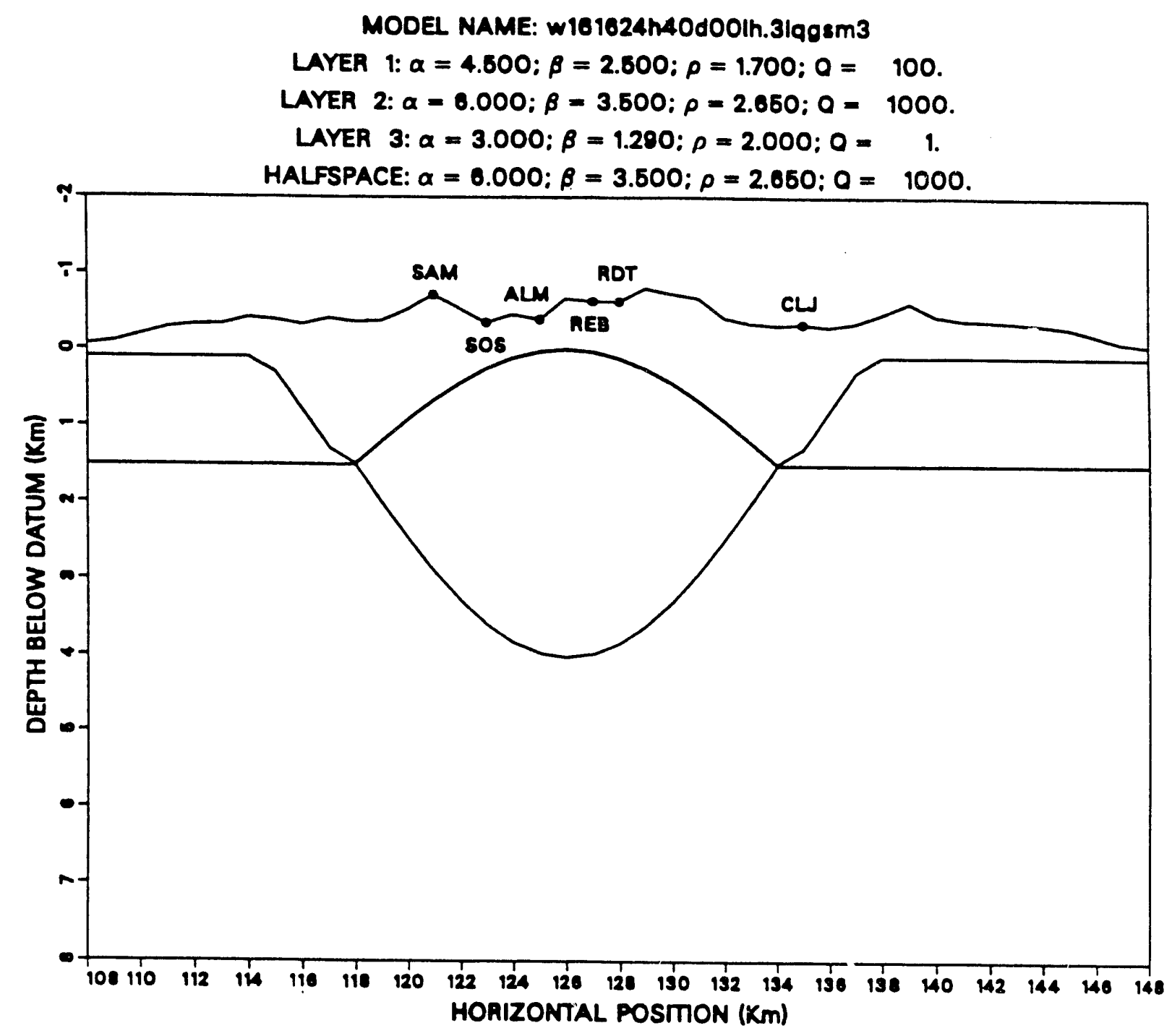

Figure 5.1 Model structure which yielded the best fit to the 4-event averaged Kuril Islands data shown in Chapter 2. All depths are relative to the arbitrary zero datum shown. Material parameters for each layer are listed at the top. Layer 1 is the upper caldera fill. Layers 2 and the halfspace are basement granite. Layer 3 is the low $Q$ zone. The name of the model is also listed at top for reference. 
VERTICAL COMPONENT SPECTRAL RATIOS

OBSERVED: KURIL ISLANDS (4 EVENTS) rocordOd in VALLES CALDERA (ARRAY 2)

SYNTHETIC: modol $=$ w161624h40d00lh.3lagsm3 $\theta_{0}=20.0 \quad \mathrm{i}$ im $=0.0000$

$\sigma_{\text {MOO }}^{2}(100)$ : Vertical $=0.344 \mathrm{E}-01$ Totol $=0.486 \mathrm{E}-01$

Veriance Reduction (HHS): Vertical $=80.7 \%$ Total $=84.0 \%$
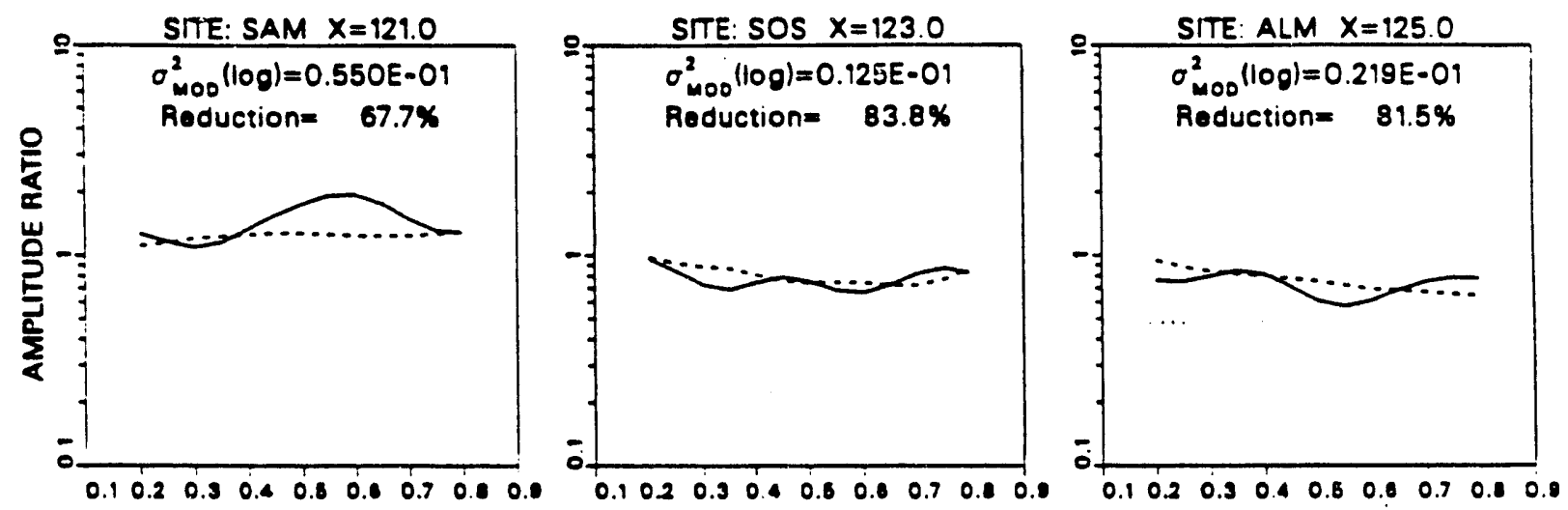

OBSERVED
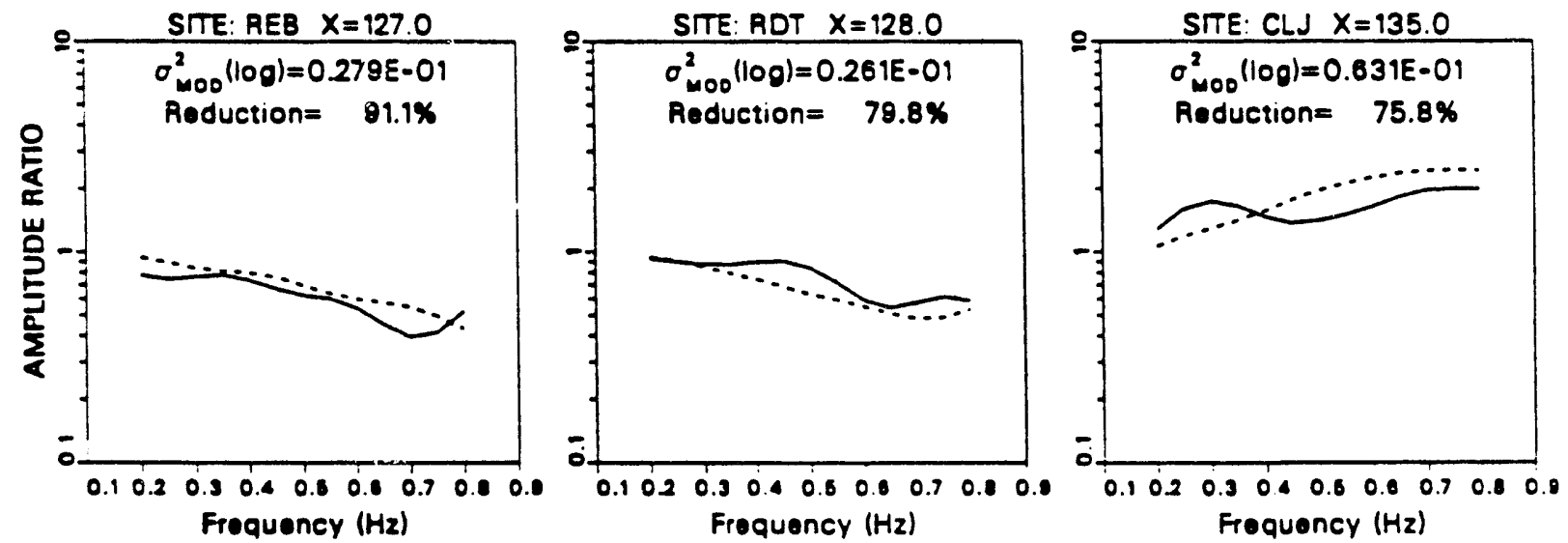

Figure 5.2a Vertical component spectral ratios for the synthetic Aki-Larner displacement solutions for the model in Figure 5.1 (dashed lines) compared with the observed ratios for the 4-event averaged Kuril Islands data (solid lines) shown previously in Chapter 2. Incidence angle used was $\theta_{0}=+20^{\circ}$. Residual variance and variance reduction are shown in each sub-plot for each site. The total over all sites and over all results for both components are listed at the top of the figure. 
HORIZONTAL COMPONENT SPECTRAL RATIOS

OBSERVED: KURIL ISLANDS (4 EVENTS) rocordUd in VALLES CALDERA (ARRAY 2)

SYNTHETIC: model $=$ W161624h40d00Ih.3lagsm3 $\theta_{0}=20.0 f_{1 M}=0.0000$

$\sigma_{\text {mOD }}^{2}(100):$ Horizontal $=0.627 \mathrm{E}-01$ Total $=0.486 \mathrm{E}-01$

Varience Reduction (HHS): Horizontal $=85.4 \%$ Total $=84.0 \%$
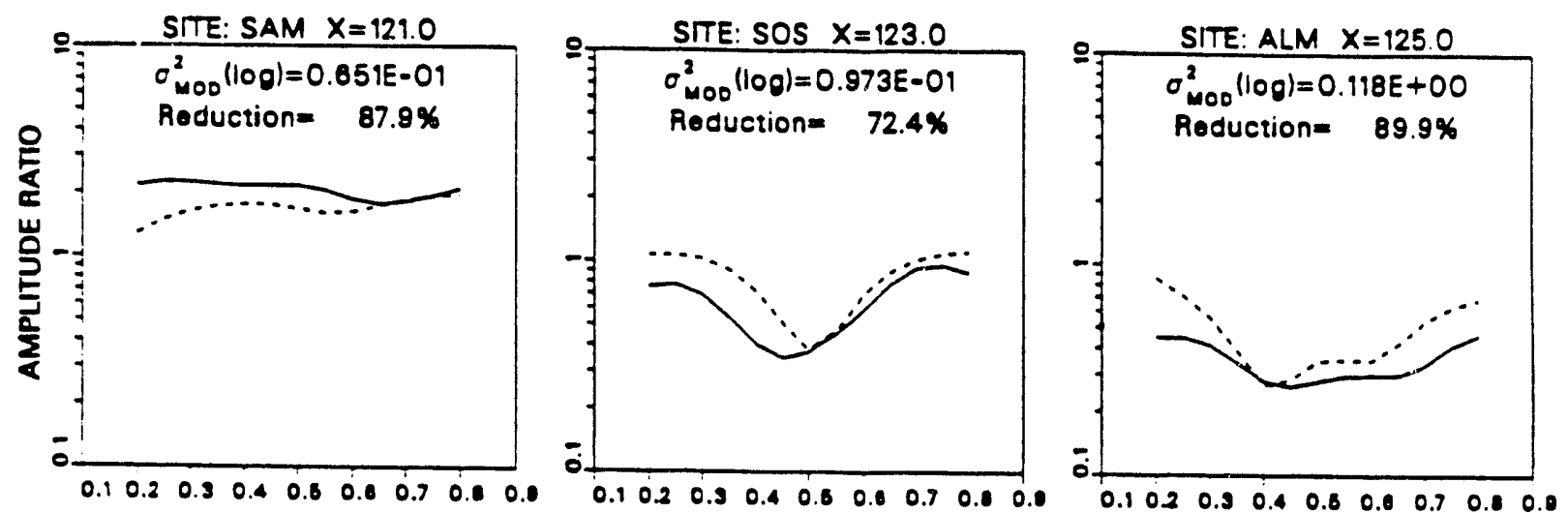

OBSERVED

. SYNTHEETC..
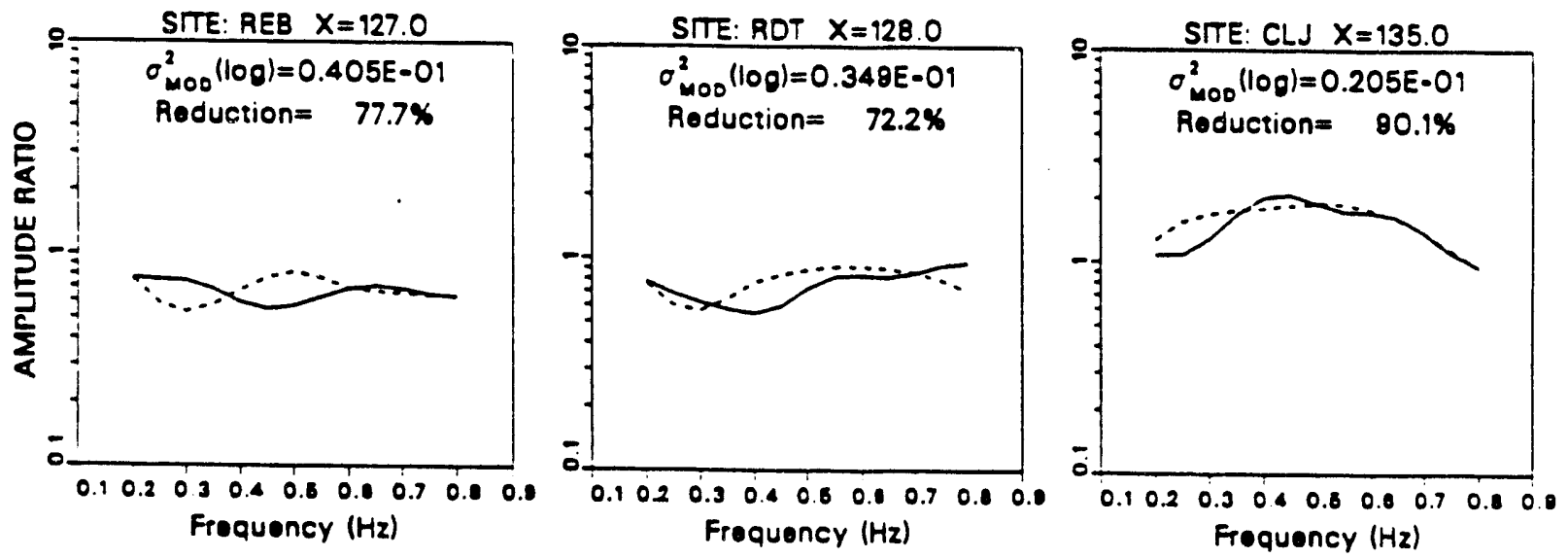

Figure 5.2b Same as Figure 5.2a except the results for the horizontal components are shown. 


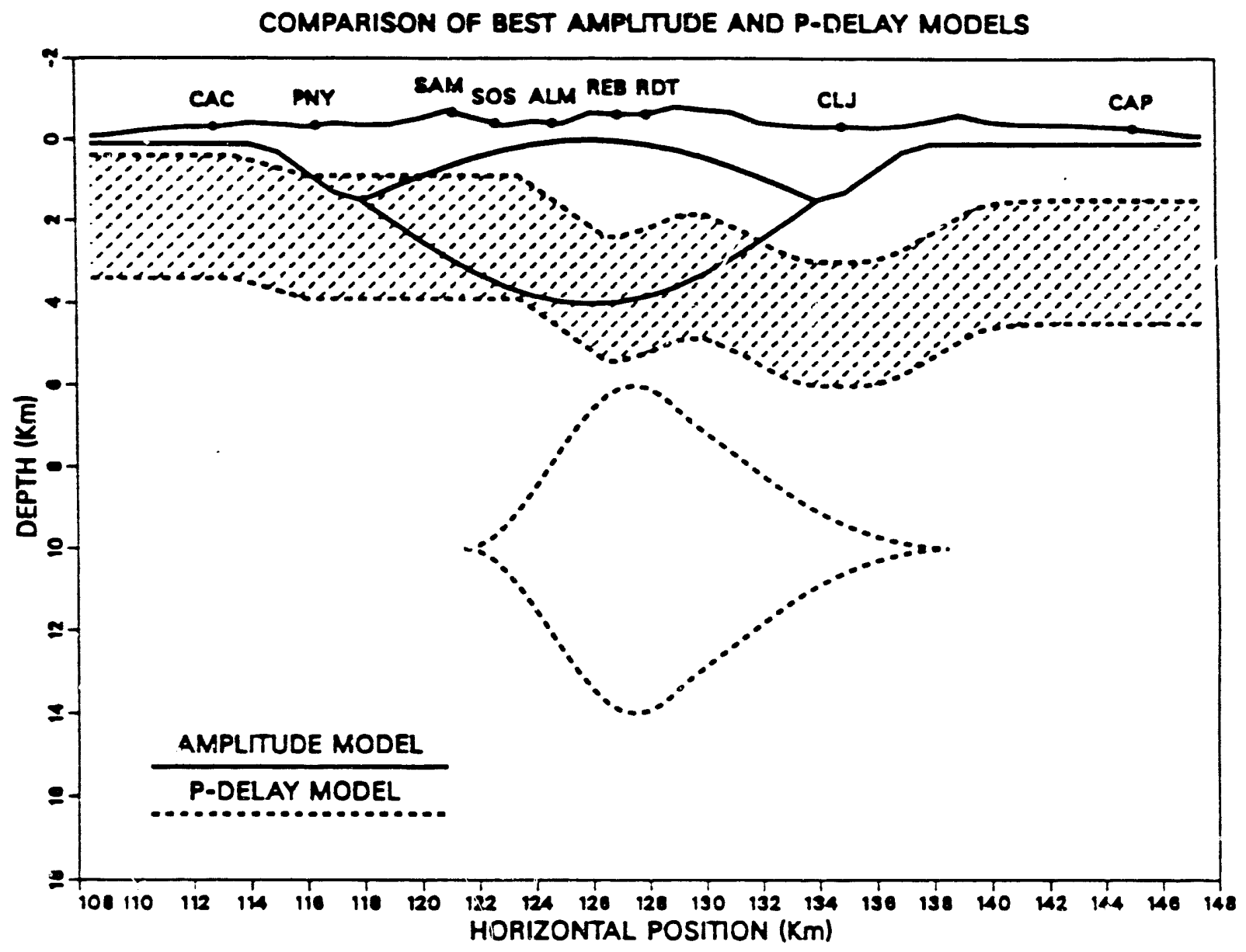

Figure 6.1 Direct comparison of the two best mociels obtained from the modeling efforts in Chapters 4 add 5. Solid lines show the best amplitude model and dashed lines show the best ray model. The iatched area shows the range of ambiguity in the overall depth of the caldera fill interface for the ray model. 
Further studv on uniqueness problem in seismological delineation of a magma chamber.

In last year's progress report, we used a new seismogram synthesis method called "Boundary Integral-Gaussian Beam Method" in studying the wave field observed on the surface of a half-space containing a magma chamber. The wave fields inside and outside the chamber are expressectus superpositions of Green's functions generated at a finite number of properly distributed point sourctis and the intensity of each source is determined to match boundary conditions at the free surface and the surface of chamber.

We were surprised by the similarity of wave field observed at the free surface between the case of low velocichamber and that of hivelocity chamber. At thar time, we computed spectral amplitutes only atselected frequenoses. In the past year, we developed a computer progran to $c$ alate the time-domain solution by applying Fourier transform to the frequency resqonse. the domain solution confirmed our earlier finding more convincingly as shown in Figs. 2 through 5. The seismograms in these figures were computed for 100 stations located along the free-surface, covering a distance equal to 8 times the radius a of the chamber. The center of the array is immediately above the center of the chamber. The source is a vertically incident plane wave of Ricker wavelet shape, whose characteristic frequency $f_{c}$ corresponds to selected values of $\eta$ (ETA) in the spectra shown in Figure 1 (a), (b), according to:

$$
\mathrm{f}_{\mathrm{c}}=\frac{\mathrm{v}}{2 \mathrm{a}} \eta
$$

where $v$ is the shear wave velocity in the half-space. The total duration of each seismogram is 90 seconds. The time axis in all figures is normalized by $\mathrm{a} / \mathrm{v}$, so that 10 units represent a duration of $10 \mathrm{a} / \mathrm{v}$ seconds. Figure 1 corresponds to the case of low velocity chamber, for values of velocity and density ratios of the chamber to the half-space $0.5 / 1$ and $0.67 / 1$ respectively; and depth of the center of the chamber $\mathrm{H}=2 \mathrm{a}$. In (a) the characteristic frequency $f_{c}=v / 2 a(\eta=1)$. A prominent feature observed in this seismogram section is the strong attenuation of the incident wave at stations located in the region $|x| \leq 2 a$ on the 
surface. Also we observe a delay of about 1 time unit of the waves transmitted through the chamber which reach the genion $|x| \leq a$ : Outside this region the transmitted waves interfere with the waves reflected at the boundary of the chamber. The later arrivals can be associated with resonance butit by trapped waves inside the chamber, as described in last year's report. These wavestipeat regularly after 2 times units and their amplitude decreases in about $50 \%$ each time. Referring to Figure 1(a), these pulses due to resonance correspond to the peak value of the amplitude spectrum observed at $n=1$.

In $(b), f_{c}=v / a(\eta=2)$ and we can observe roughly the same features described for (a). We must point out here that the amplitudes in both (a) and (b) are normalized to 1, i.e. divided by the response of the raff-space without the chamber to an incident wave of amplitude 1. In Figure 3(a), $(\mathrm{b})$, he depth of the center of the chamber was inereased to $\mathrm{H}=4 \mathrm{a}$. The same featura described for Figure 2 are observed in general, but here the resonance pulses are separat about 4 time units and the wavefronts are more curved. The larger time elapsed between pulses suggests the contribution of the freesurface effect to the build-up of resonance modes inside the chamber.

The case of a high velocity chamber is shown in Figure 4(a), (b). In contrast with the low velocity case, here the velocity and density ratios are $2 / 1$ and $1.5 / 1$ respectively. The depth is kept at $H=2 a$. In (a) $f_{c}=v / 2 a$. The similarity of the attenuation pattern observed for stations above the chamber is remarkable as compared with the low velocity chamber, particularly for the region $|x| \leq a$. However, different features are the earlier arrivals of the waves transmitted through the chamber, that reach stations within $|x|<a$, and the absence of resonance pulses. The latter is in agreement with the fact that most of the corresponding amplitude spectrum shown in Figure $1(b)$ is flat and smooth. In the region $|x|<a$ it is possible that diffracted waves originated at points $(-a, H)$ and $(a, H)$ on the boundary of the chamber arrive earlier than the incident wave (Nolet, 1988).

In $(b) f_{c}=v / a$. Here the earlier arrivals are observed more prominently, particularly for the region $|x|<2 a$. Figure 5 corresponds to the case where the depth of the high velocity 
chamber is $H=4 a$. In (a) $f_{c}=v / 2 a$, we observed that the region of strong attenuation extends to $|x|<2 a$, becoming more similar to the attenuation pattern shown in $5(a)$. The region of earlier arrivals extends as well, to $|x|<5 \mathrm{a} / 2$. resonance pulses are observed. In (b) $\mathrm{f}_{\mathrm{c}}=\mathrm{v} / \mathrm{a}$, and we can obctrved the same features described for (a). Although details of wave-forms are somewhethefferent, the general pattern of wave field observed at the surface due to vertically incident $\mathrm{SH}$ waves is very similar between the case of low velocity chamber and that of high velocity chamber. Of course, the first arrivals are eartier for the latter case than for the former, but the strong attenuation of main part of the signal at the surface directly above the chamber is observed equally for both cases.

This is a very ironical situation for seismologists who have been trying to go beyond the travel time tomograthy to the wave-form inversion for delineating the subsurface structure, because this eximple strongly suggests that it is better to restrict the data to the first arrival rather than including the whole waveform for a unique inversion. It is clear, at least for this example, that the linearized inversion based on the first Born approximation, the approach taken by most seismologists so far, would never work.

This shocking finding, however, does not affect the conclusion we obtained for the Valles Caldera in the preceding section. First, we avoided the formalism of linearized inversion and instead relied on the variance reduction for many trial forward non-linear modelings. Secondly, we used primarily the first $\mathrm{P}$-wave time data for the determination of velocity structure, and the total waveform data were used mainly for delineating the seismic Q-structure. 


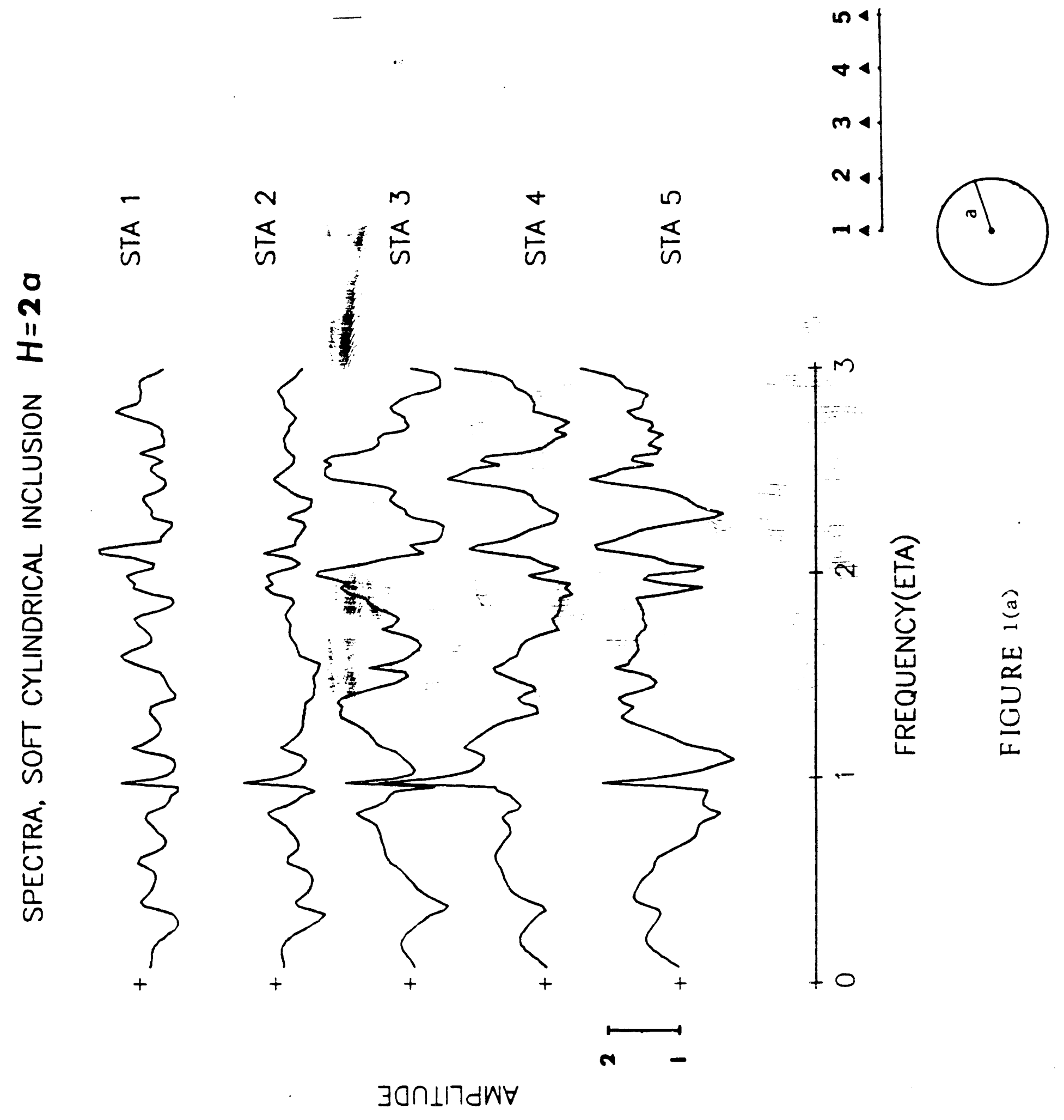




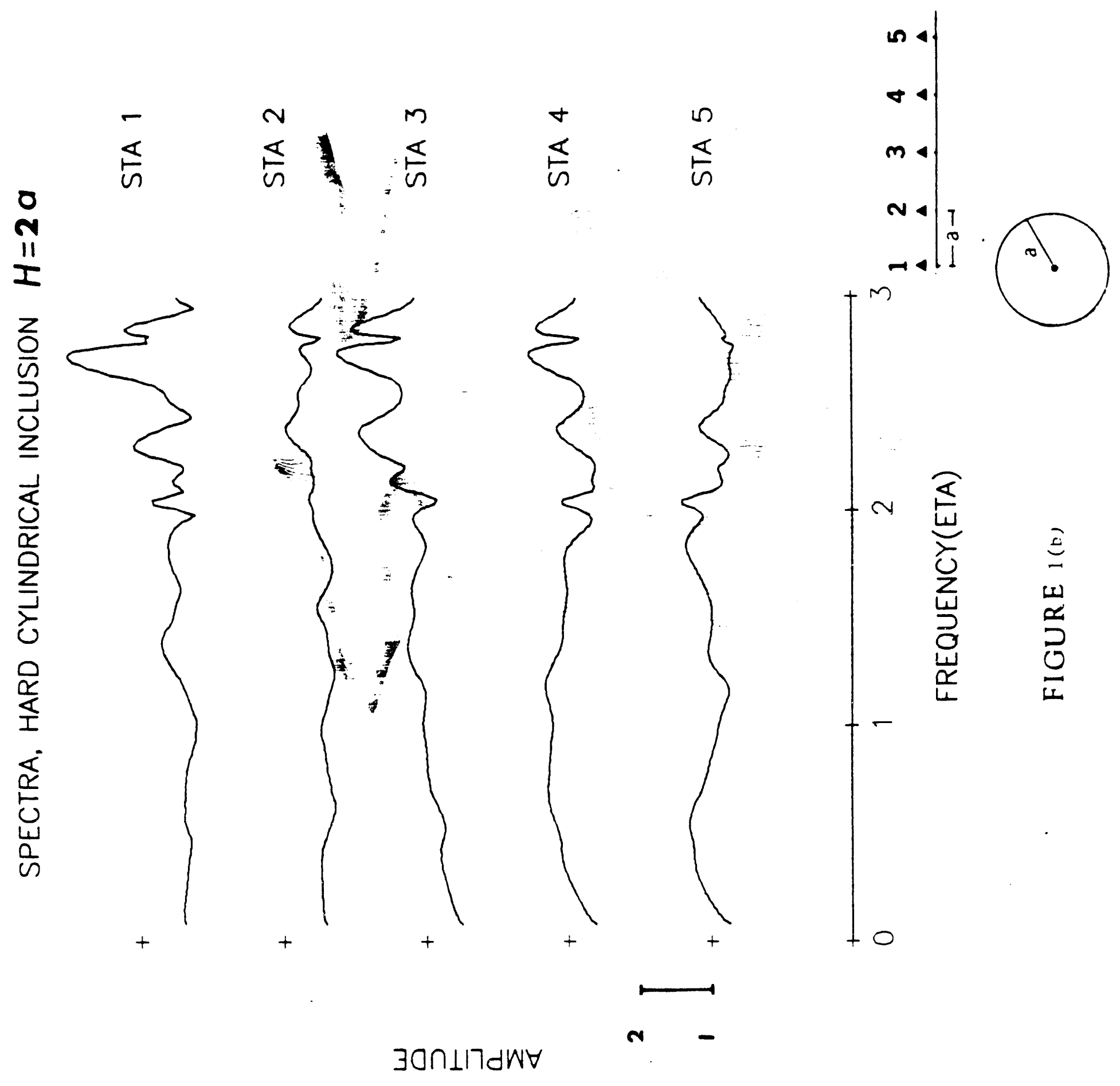




\section{LOW VELOCITY INCLUSION}

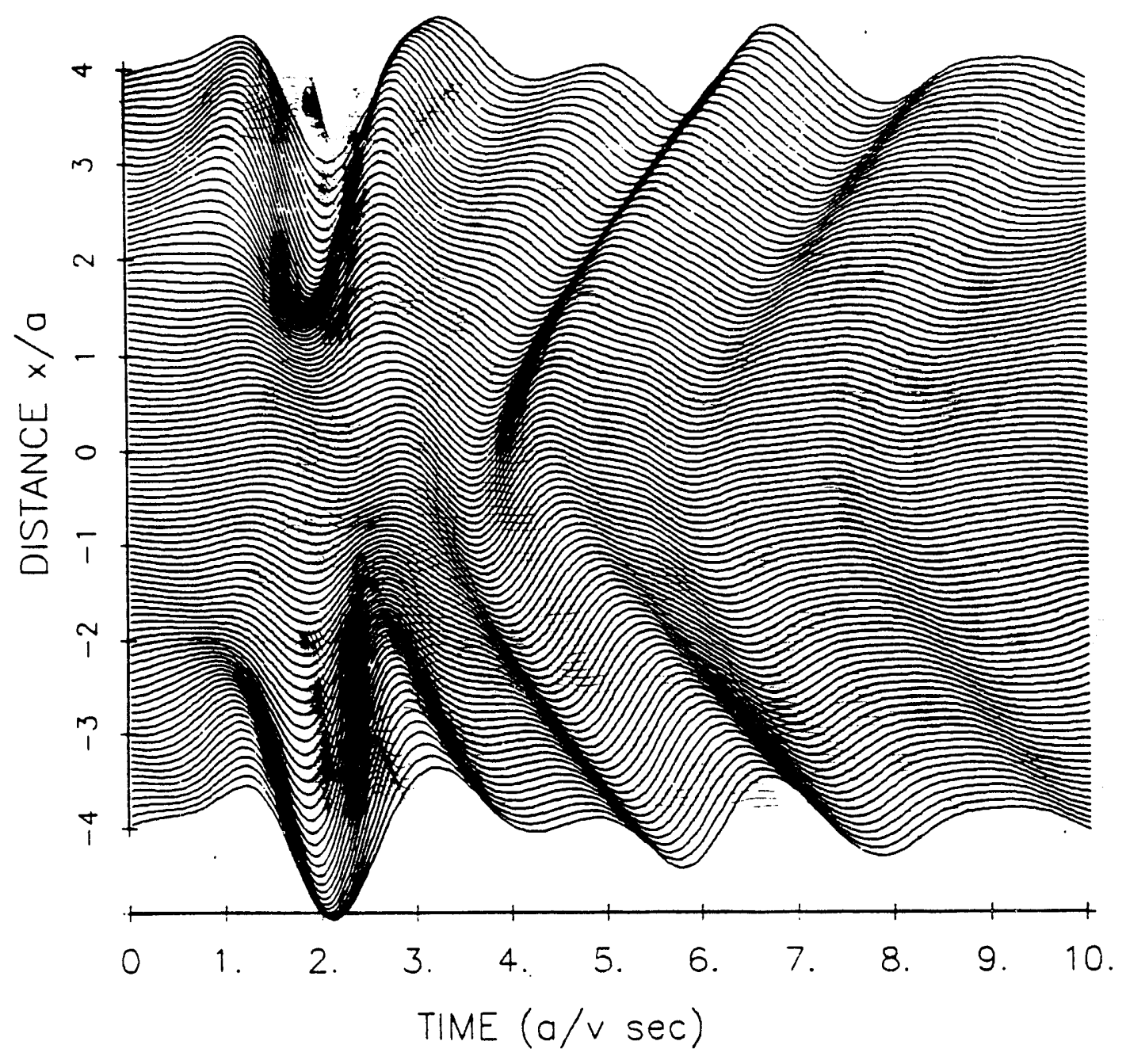

$$
\begin{aligned}
& v_{I}=0.5 \mathrm{v} \\
& \rho_{I}=0.67 \rho \\
& I_{C}=\mathrm{v} / 2 \mathrm{a} \\
& H=2 a
\end{aligned}
$$

FIGURE 2(a) 
LOW VELOCITY INCLUSION

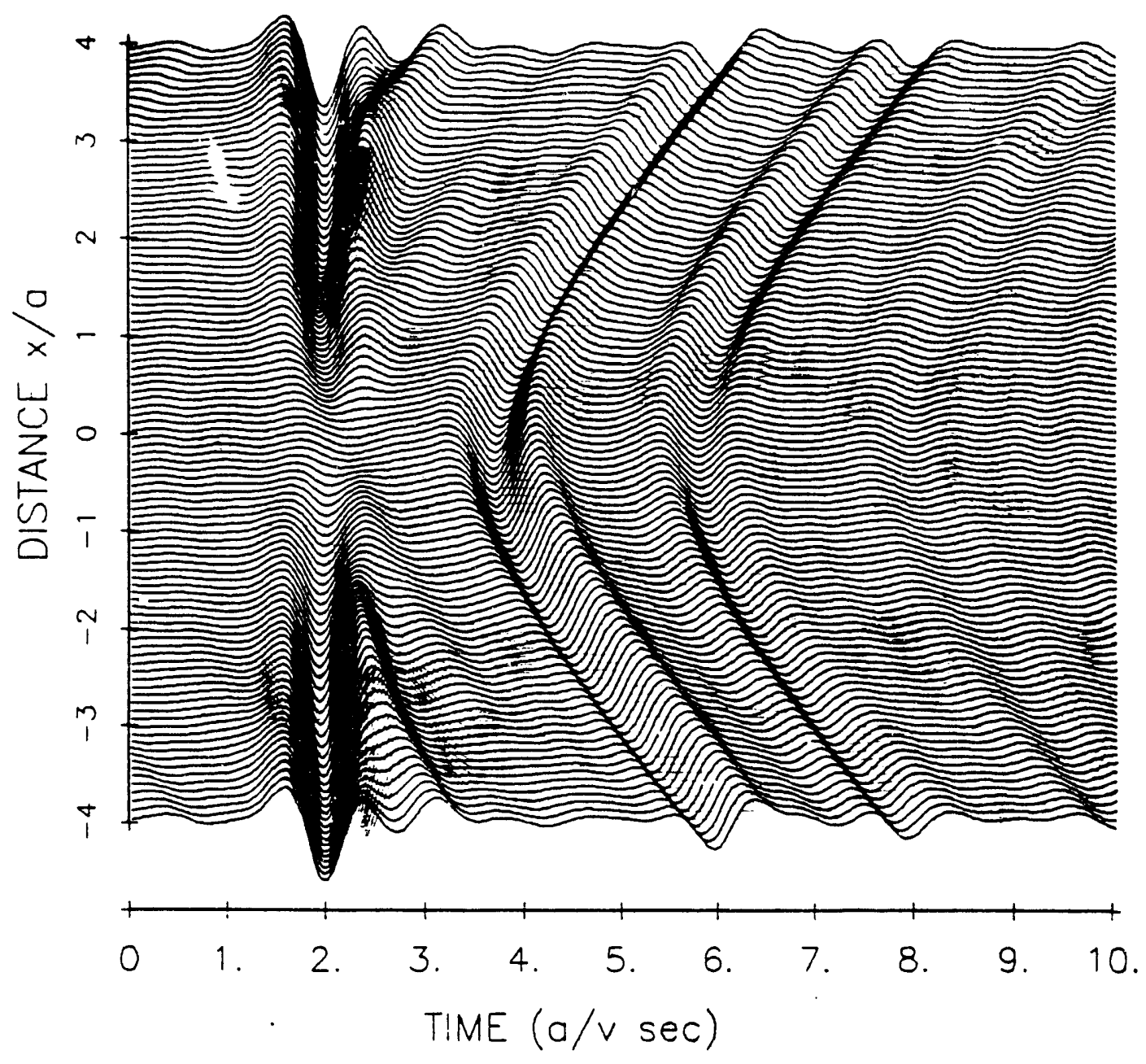

$$
\begin{aligned}
& v_{I}=0.5 v \\
& \rho_{I}=0.67 \rho \\
& I_{C}=v / a \\
& H=2 a
\end{aligned}
$$

FIGURE 2(b) 


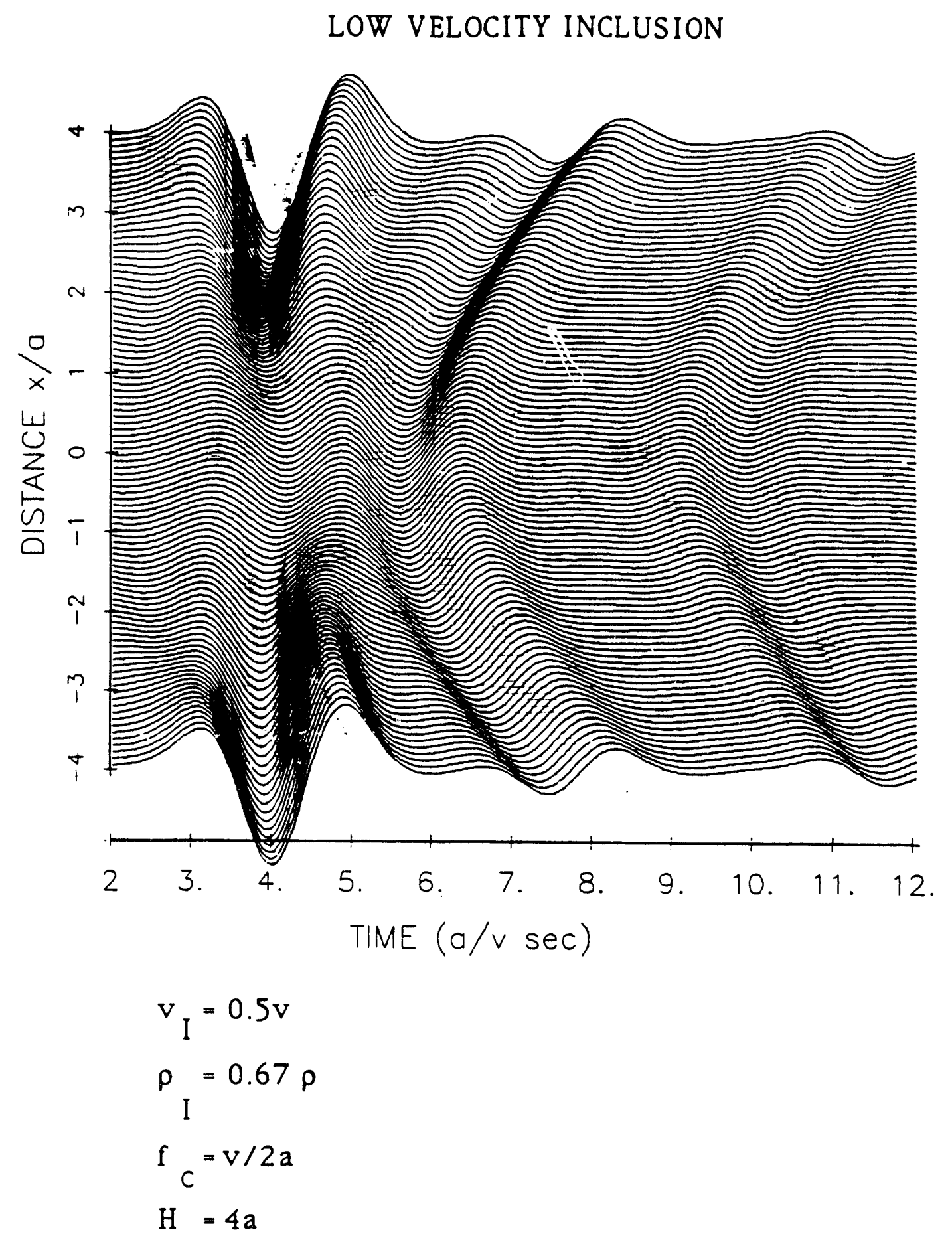

FIGURE 3(a) 


\section{LOW VELOCITY INCLUSION}

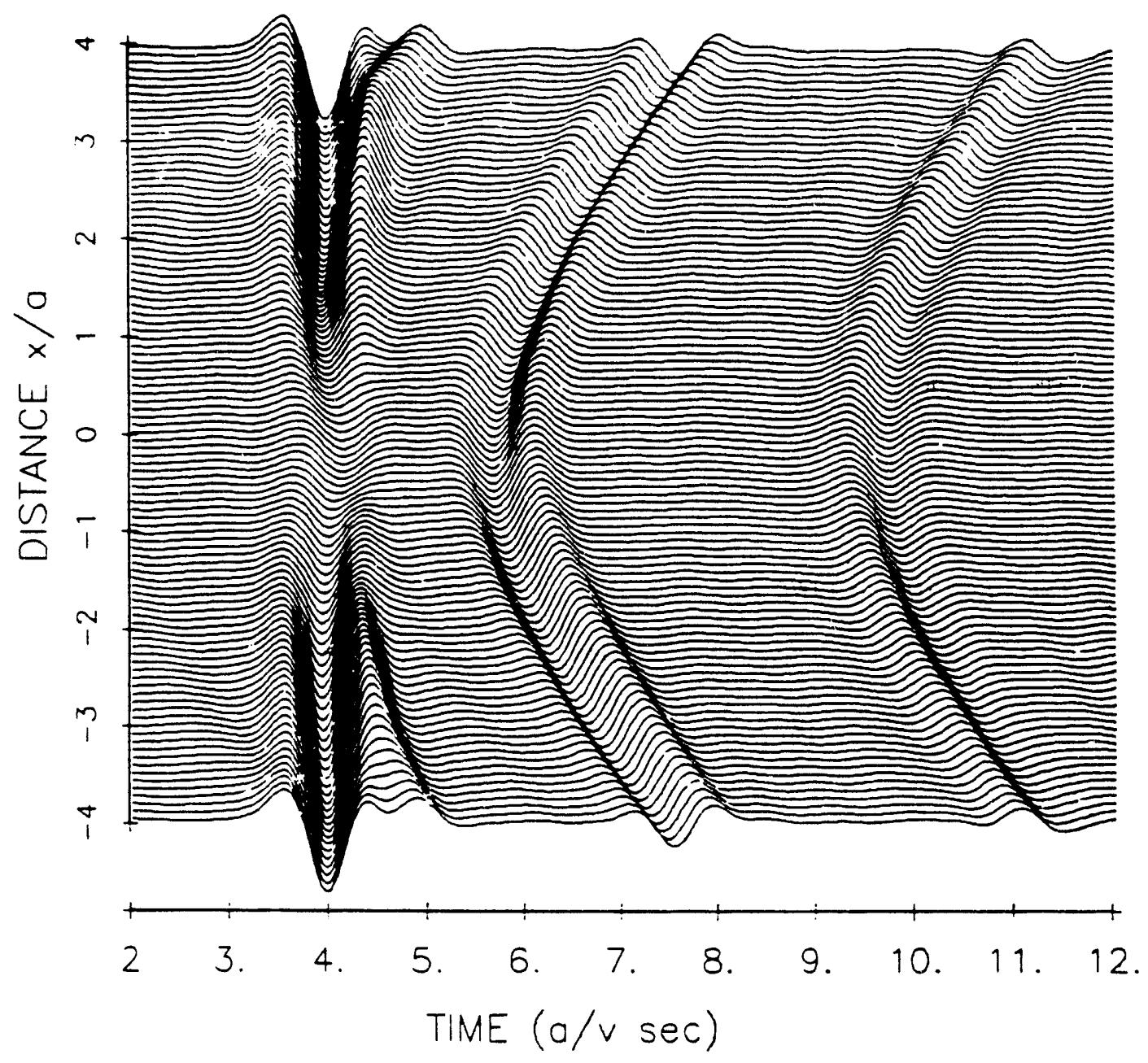

$$
\begin{aligned}
& v_{I}=0.5 v \\
& \rho_{I}=0.67 \rho \\
& f_{C}=v / a \\
& H=4 a
\end{aligned}
$$

FIGURE 3(b) 


\section{HIGH VELOCITY INCLUSION}

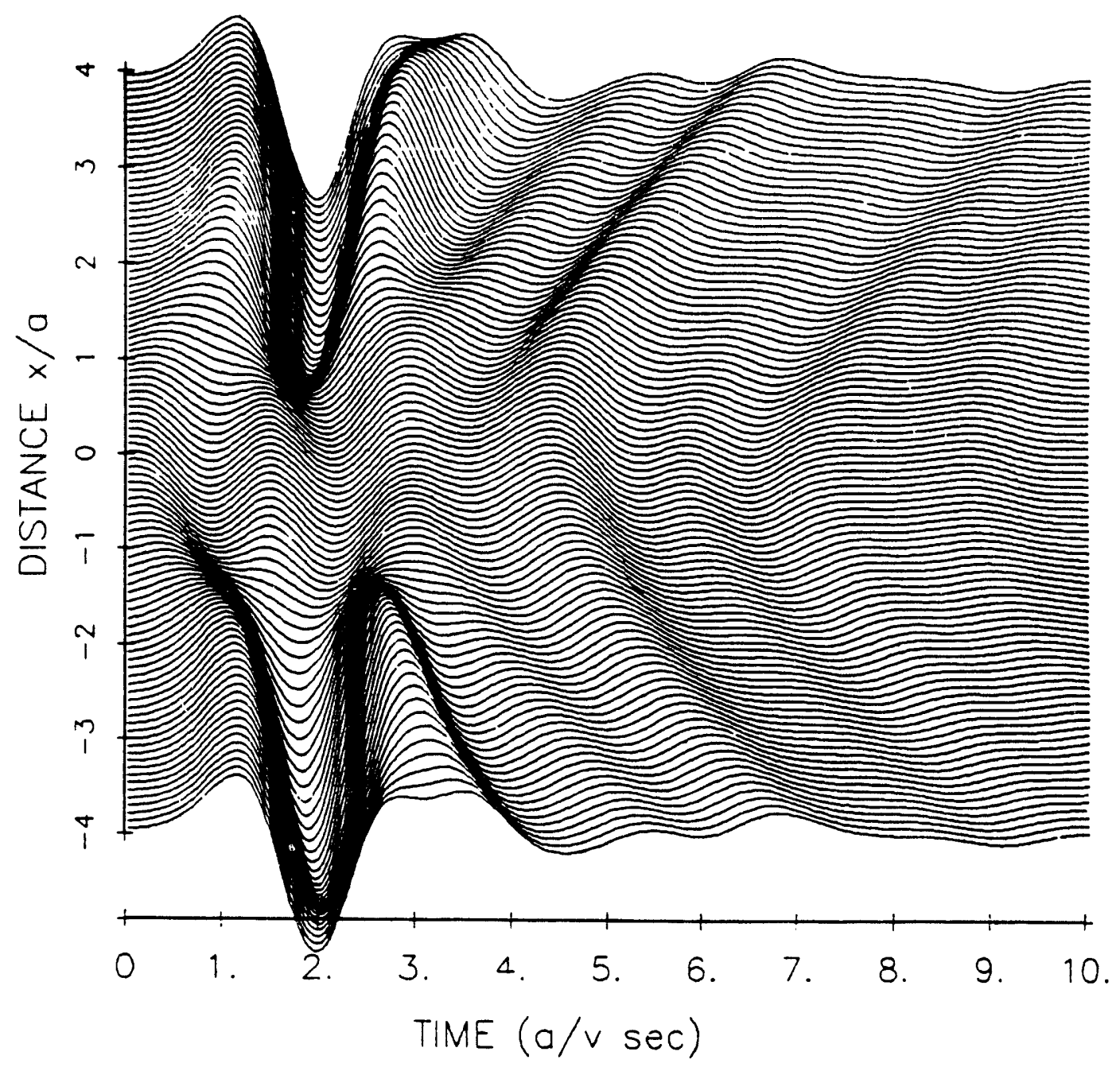

$$
\begin{aligned}
& v_{I}=2 v \\
& \rho_{I}=1.5 \rho \\
& f_{C}=v / 2 a \\
& H=2 a
\end{aligned}
$$

FIGURE 4(a) 


\section{HIGH VELOCITY INCLUSION}

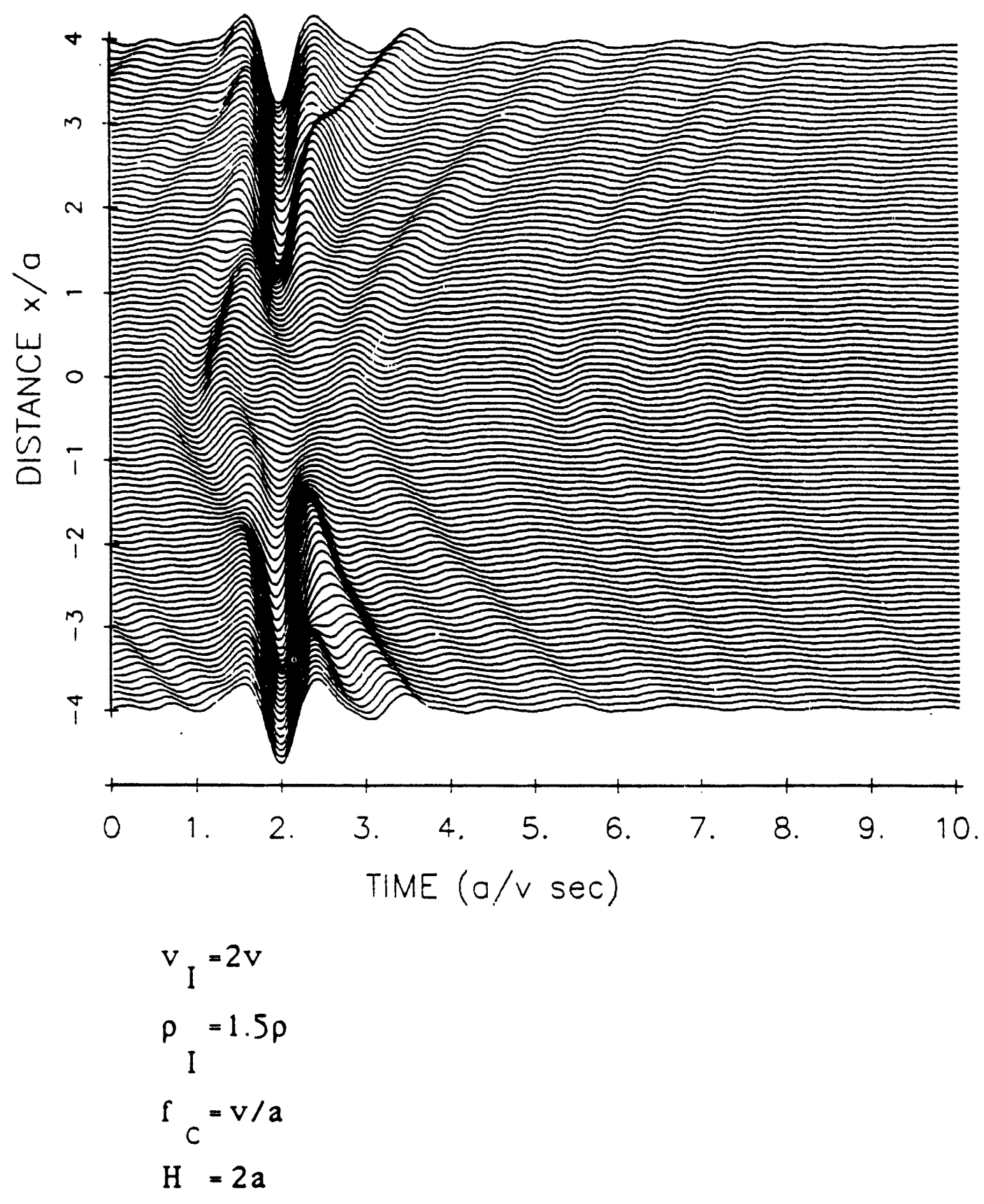

FIGURE 4(b) 


\section{HIGH VELOCITY INCLUSION}

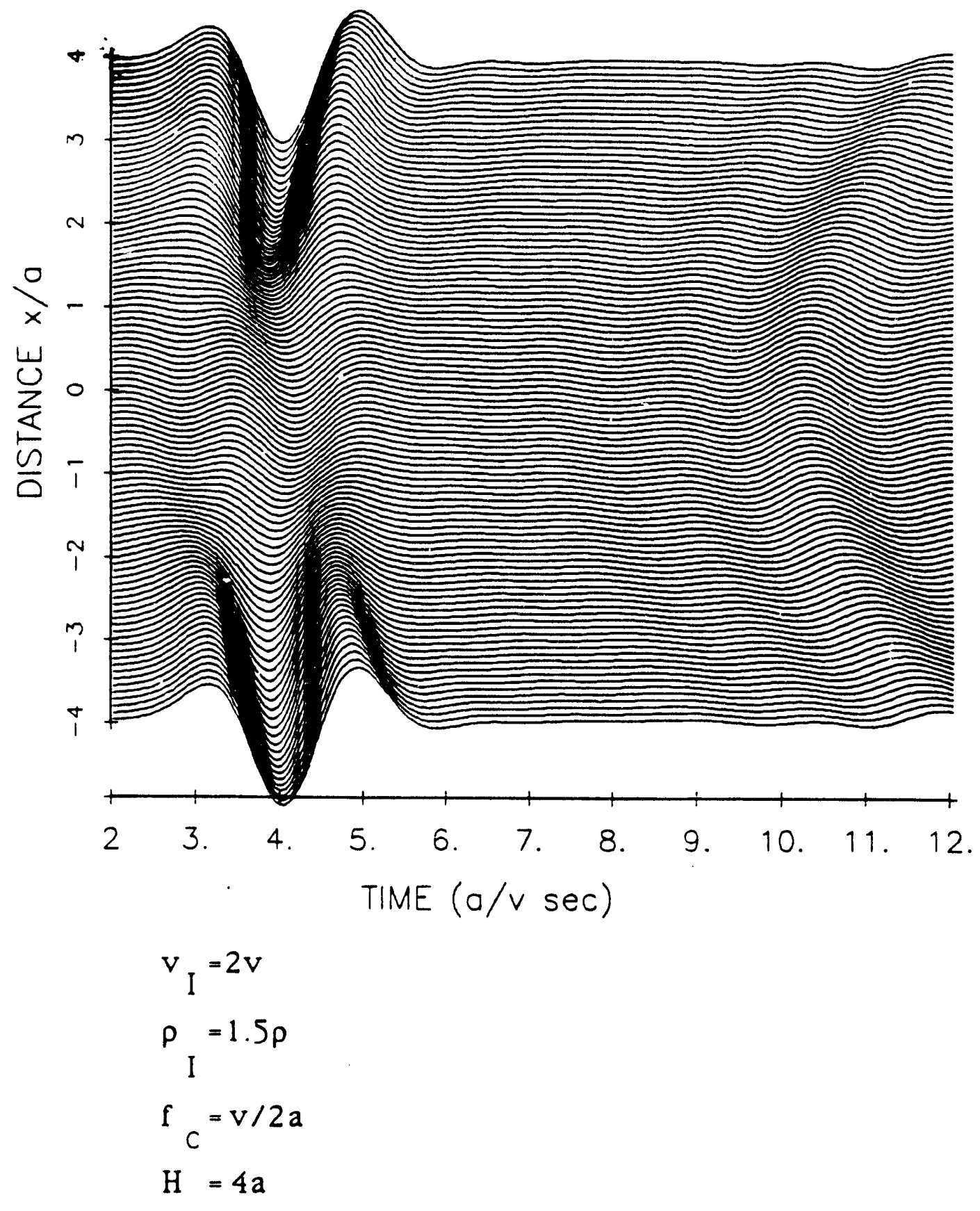

FIGURE 5(a) 
HIGH VELOCITY INCLUSION

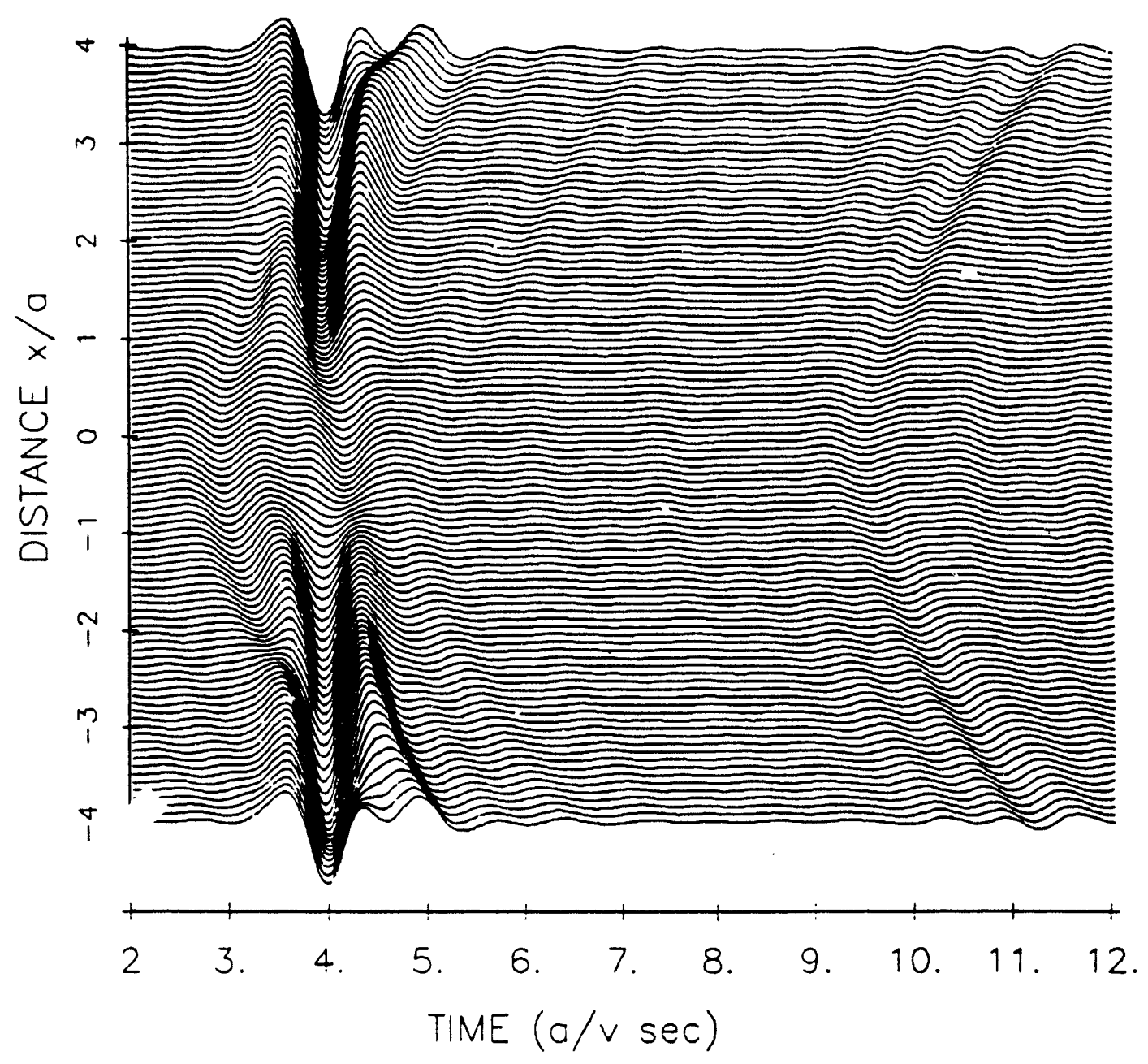

$$
\begin{aligned}
& v_{I}=2 v \\
& \rho_{I}=1.5 p \\
& f_{C}=v / a \\
& H=4 a
\end{aligned}
$$

FIGURE 5(b) 
(4) Fracture related seismic attenuation in VSP data.

As described in detail in the Ph.D. thesis of Y.G. Li as we" as several published papers listed earlier, we have studied effects of aligned cracks in the earth on the shearwave splitting and the P-wave polarization observed by our VSP experiments at Oroville (in the neighborhood of an active normal fault zone), at Hi Vista (near the San Anoreas fault), and at the Cajon Pass scientific deep drilling site. We were able to define quantitatively the distribution of crack density using ray tracing equations of Cerveny (1972) for anisotropic heterogeneous media and Hudson's $(1981,1982)$ elastic constants for a medium containing aligned cracks. Our results, in general, agreed with those obtained by other available methods such as borehole televiewer (Moos et al, 1983) and hydraulic fracturing method (Zoback et al, 1980; Hickman et al, 1988).

We found that the maximum crack density in the Oroville fault zone was $8 \%$ with the crack plane parallel to the fault plane, and average crack density in granite at Hi Vista was $3.5 \%$ with vertical crack plane aligning $\mathrm{N} 30^{\circ} \mathrm{W}$, and a population of a cracks was aligned $N 70^{\circ} \mathrm{E}$ in the depth range between 1300 and $1800 \mathrm{~m}$ at Cajon Pass with an average crack density of 2 to $3 \%$.

We also studied the attenuation of seismic waves using the VSP data obtained at the above three sites.

The three-component VSP data obtained from the Hi Vista borehole site show coherent waveform for the first arrival as shown in fig. 2 , where three columns correspond to three vibrator sites with offset $100 \mathrm{~m}$ from the wellhead in the south (left), southeast (middle) and east (right) directions, respectively, and three rows correspond to three types of waves: SH (upper), SV (middle) and P (lower). Each panel contains eighteen seismograms recorded at depths from $100 \mathrm{~m}$ to $600 \mathrm{~m}$ at an equal spacing and then rotated into three mutually orthogonal ray coordinates.

Except for the shear-wave splitting, the anisotropic feature of the shear-wave attenuacion was observed and interpreted to be reiated to aligned fractures in the basement 
rock. Fig. 3 shows amplitude versus depth curves for the east vibrator site. The solid curve is denoted by $\mathrm{SV}_{\text {obs }}$ for observed $\mathrm{SH}$-waves. Amplitudes are plotted in the logarithmic scale. In this case, amplitudes of SV-waves polarized trans vertical crack plane decrease faster than $\mathrm{SH}$-waves polarized parallel to the crack plane when they travel through the fractured medium vertically. We also computed ray amplitudes of two types of $\mathrm{S}$-waves using twelve first-order differential equations with second-order derivatives of the Christoffel tensor with respect to ray parameters and positions. The Christoffel tensor is formed by the wave slowness and the material elastic constants determined by the first-order ray tracing. Because two types of S-waves have different curvatures of the wavefront when they propagate in a particular direction in the anisotropic medium, they have different geometrical spreading and thus their amplitudes decay at different rates as the travelling distance increases. Calculated amplitudes versus depth for $\mathrm{SV}$-waves are plotted as a solid curve denoted by $\mathrm{SV}_{\text {cal }}$ while a dashed curve denoted by $\mathrm{SH}_{\text {cal }}$ for $\mathrm{SH}$-waves in Fig. 3. After correction of the geometrical splitting, the amplitude versus depth curve for the SV-wave is plotted by the dash-dot line while the curve for the SH-wave by the dot line in Fig. 3. The tendency of the amplitude after correction of the geometry shows still slight decreases for both types of S-waves. It is noted that the amplitude attenuation for SV-wave is larger than SH-wave. Corresponding to the dominant frequency $30 \mathrm{HZ}$, the apparent quality factor $\mathrm{Q}$ of the basement rock at $\mathrm{Hi}$ Vista borehole site is $60-80$.

The second set of VSP data taken from the Oroville borehole site shows a stronger wave attenuation in and near the fault zone. We used various seismic sources: a vertical impact source, a w.ight drop and a single air-gun in that extensive surface-to-borehole VSP experiment as shown in Fig. 4. To avoid the complexity of the source radiation and to obtain the high-frequency seismic energy we chose the data generated by the single air-gun shooting in a shallow hole $10 \mathrm{~m}$ from the wellhead for the study of attenuation. The dominant frequency of the air-gun source is about $120 \mathrm{~Hz}$. Fig. 5 shows an example of 
three component seismograms recorded at the depth of 1000 feet near the fault zone. Unfortunately, the strong $S$-waves were clipped by the instrument at the shallow depths, so we could not use the amplitude information carried by $S$-waves. Fig. 6 a shows wavetrains of $\mathrm{P}$-motion in a $64 \mathrm{~ms}$ time window recorded at 17 depth points from 200 to 1000 feet. Their amplitude spectra are plotted in Fig. 6b. Complicated waveforms recorded at the shallow depths (200 - 500 feet) may be caused by the superposition of the primarily downgoing wave and the multiples from the free surface. For simplicity, we avoided seismograms of P-waves recurded in the depth shallower than 500 feet. Fig. 7a shows the coherent waveforms in a $16 \mathrm{~ms}$ time window modified by a cosine and $7 \mathrm{~b}$ shows their amplitude spectra. The peak spectral amplitudes are shown on the right side to the corresponding seismograms in fig. 7a. After correction of the geometrical spreading obtained by ray tracing in the anisotropic medium, the spectral amplitude versus depth curve is plotted in Fig. 8a. In order to relate this to the fault zone, we plot also the spectral amplitude versus the distance from the fault plane as shown in fig. $9 \mathrm{~b}$. It shows that seismic waves encounter an obvious attenuation when they travel in and near the highly fractured fault zone. The quality facet $Q$ of the fractured rock in the vicinity of the fault zone is estimated to be $6-8$. As mentioned earlier (fig. 1), the maximum crack density was about 0.08 in the fault zone. It means that there is some close relationship between the crack density and the quality factor of the fractured rock.

From above observations at Hi Vista and Oroville, the following questions remain. (1) What are the causes of amplitude attenuation for $P$ and $S$ waves remaining after the correction for geometrical spreading? (2) Are they scattering, absorption, or both? How can they be separated? (3) Why does there seem to be a difference in attenuation between two types of $S$ waves? (4) If the difference is due to aligned cracks, can we use the observed attenuation anisotropy to define the distribution of crack? To answer these questions, we need more high-quality 3 component data and interpretation methods for separating the scattering and absorption effects. 


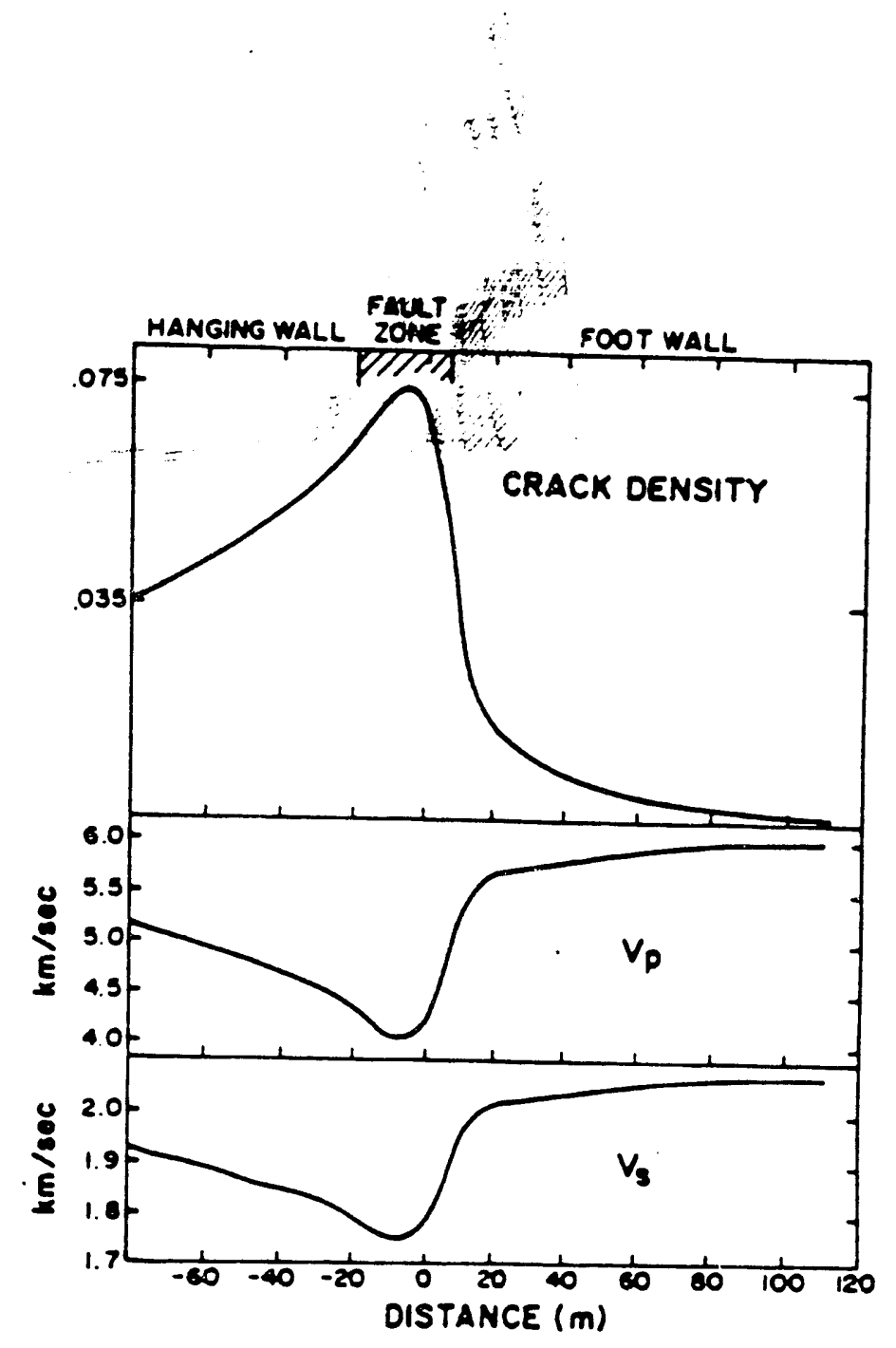

Figure 1 

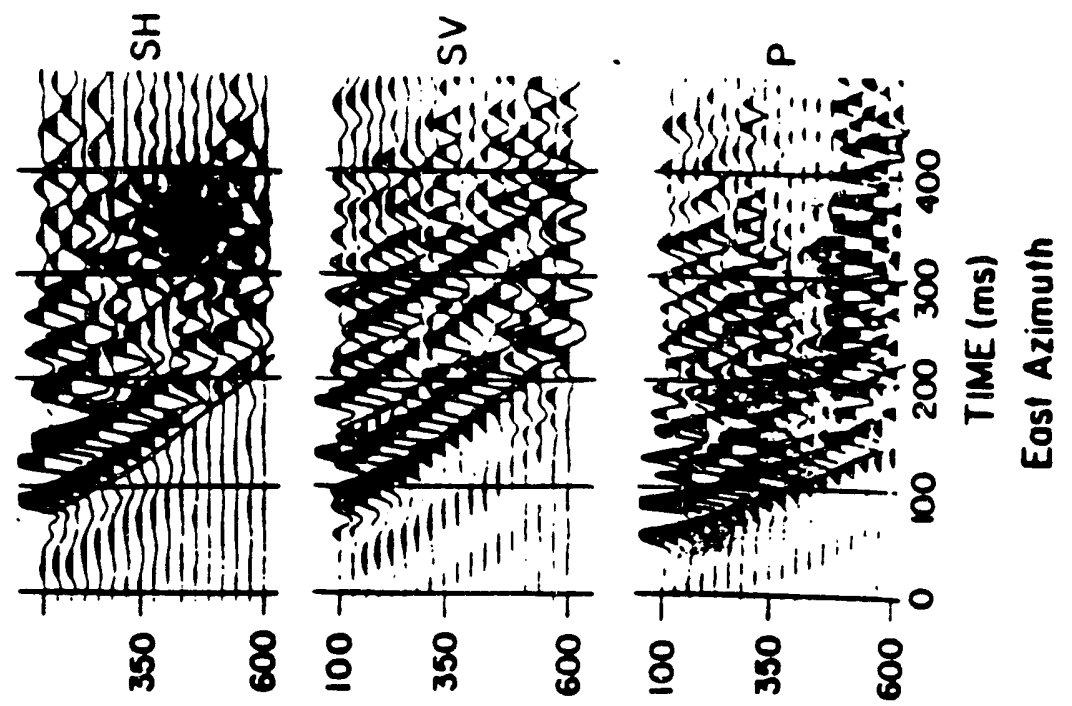

(w) $H \perp d \exists O$
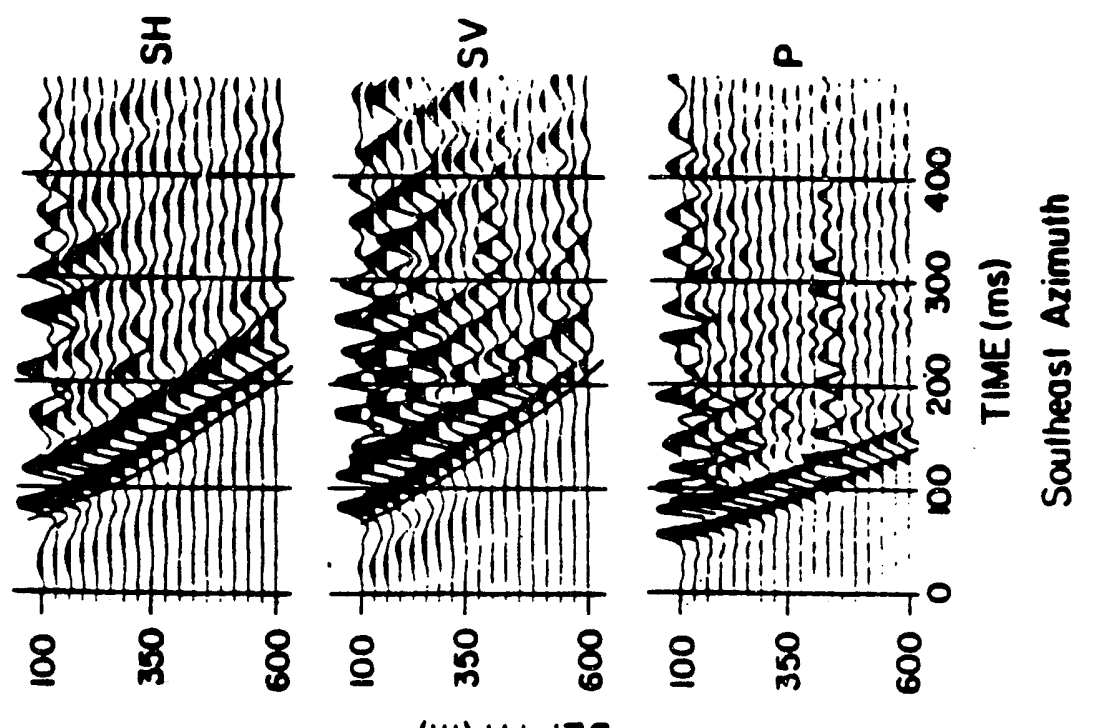

志

es
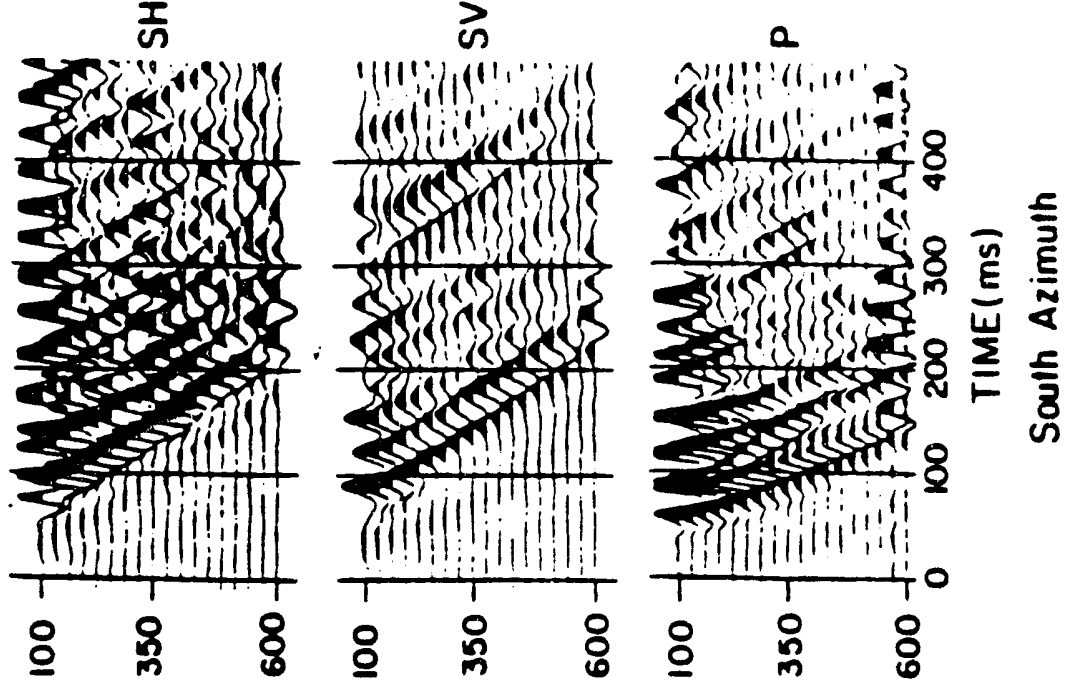

(w) $H \perp d 30$ 


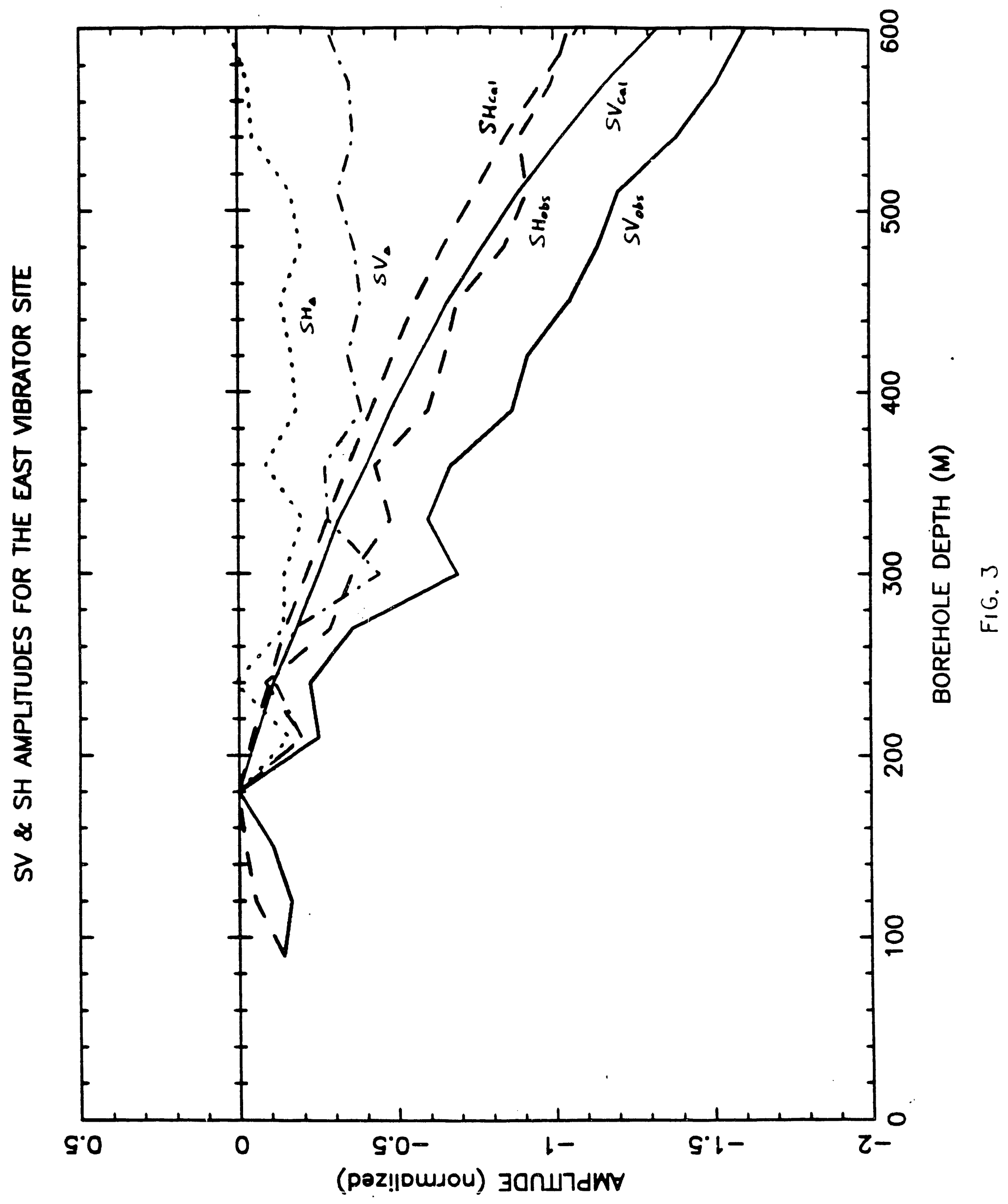




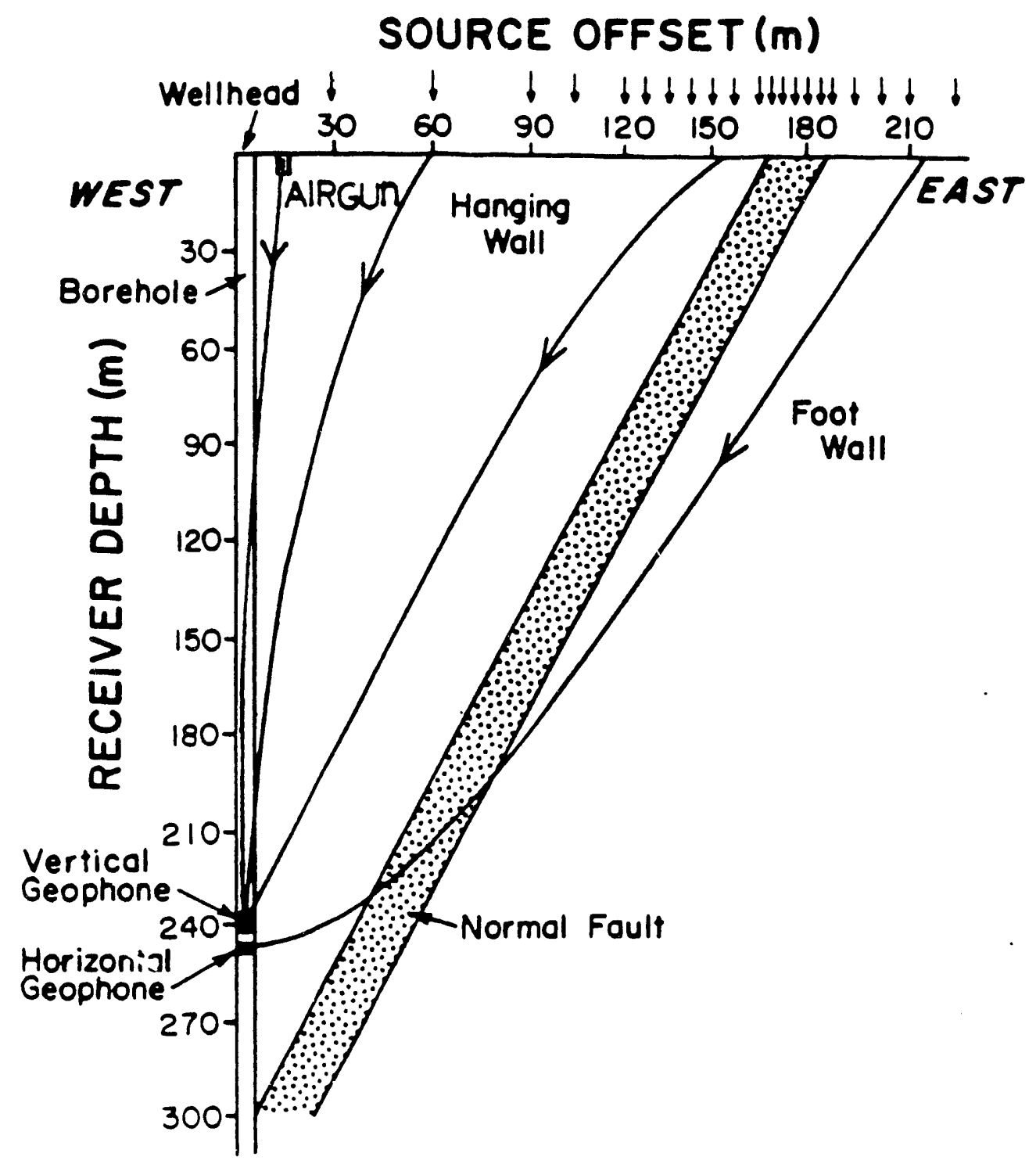

FIG. 4 


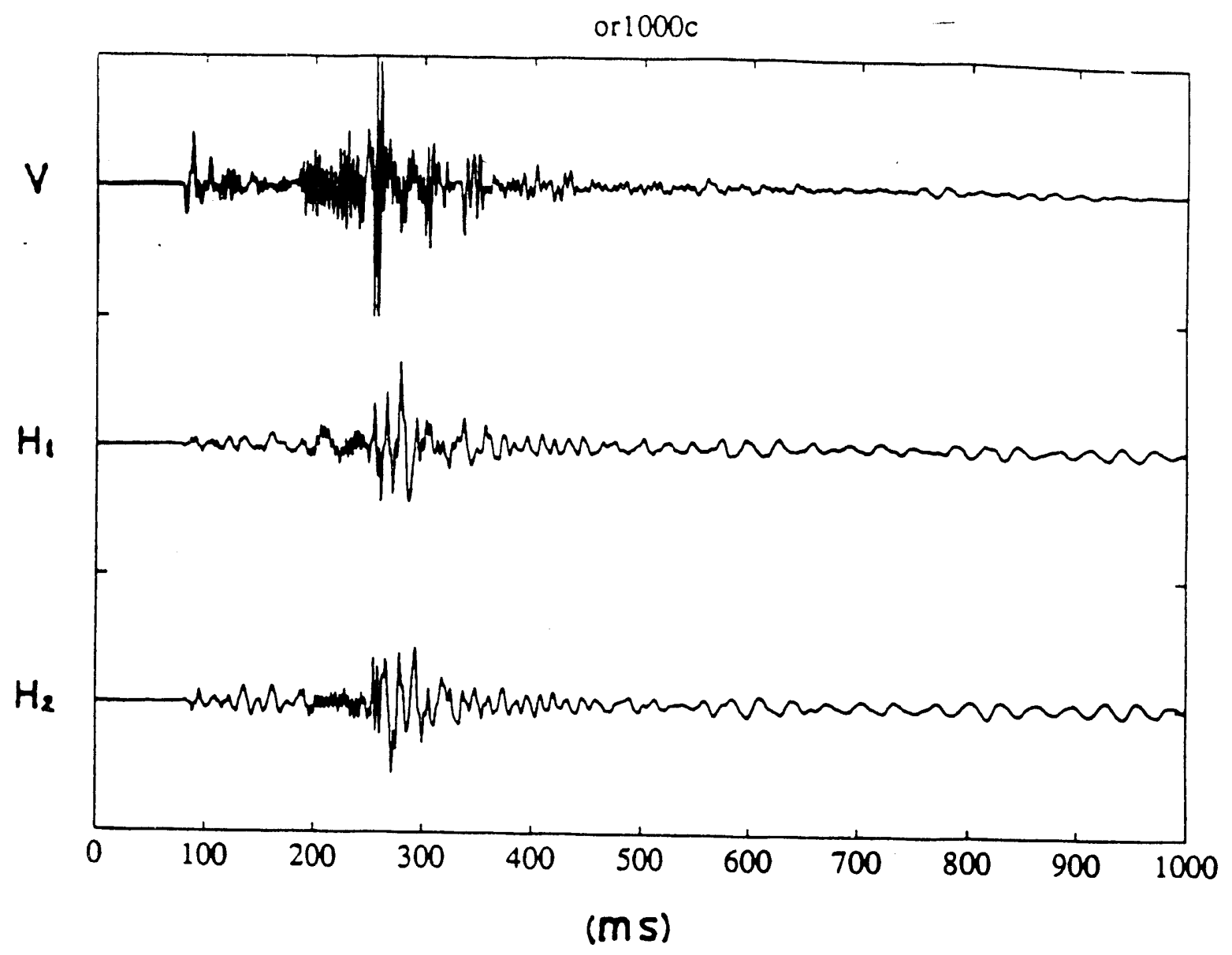

Fig.5 


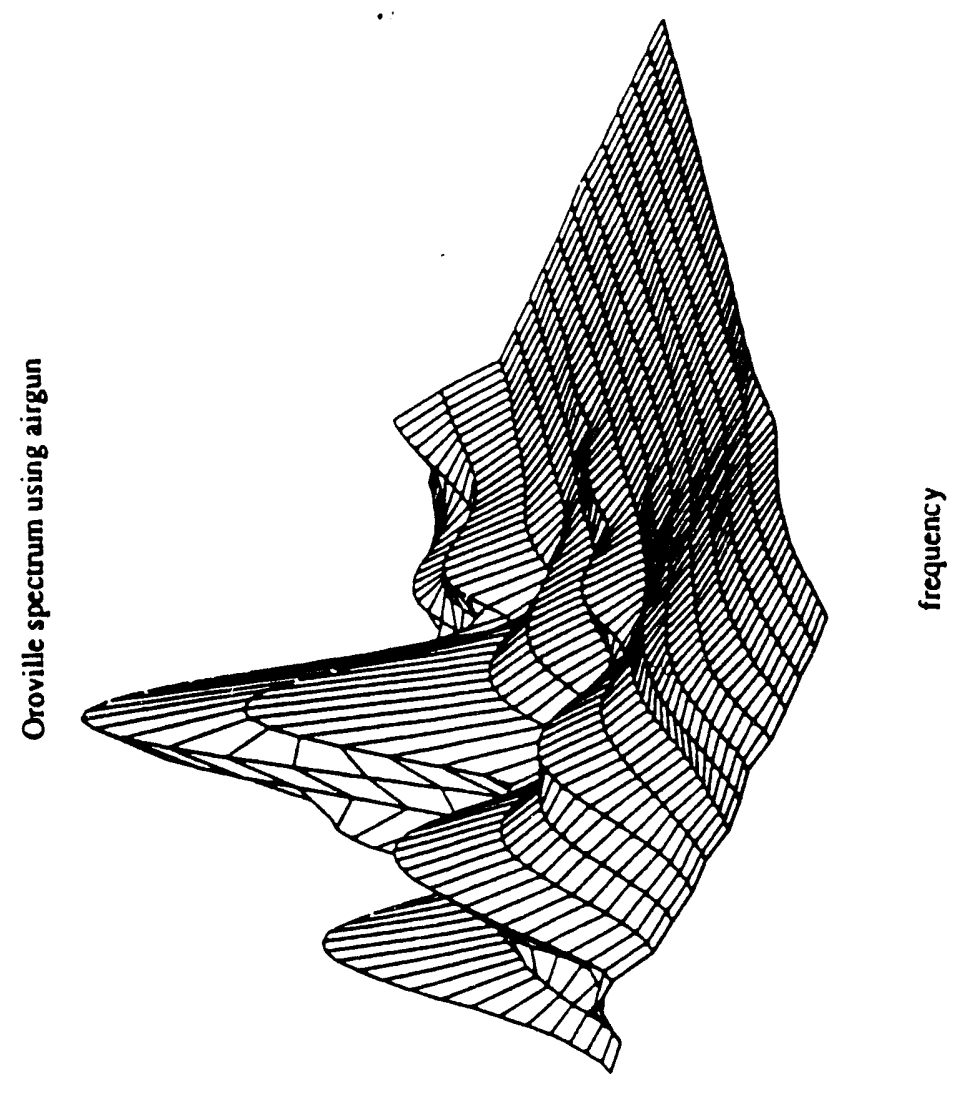

$\underline{2}$

spru!!dure

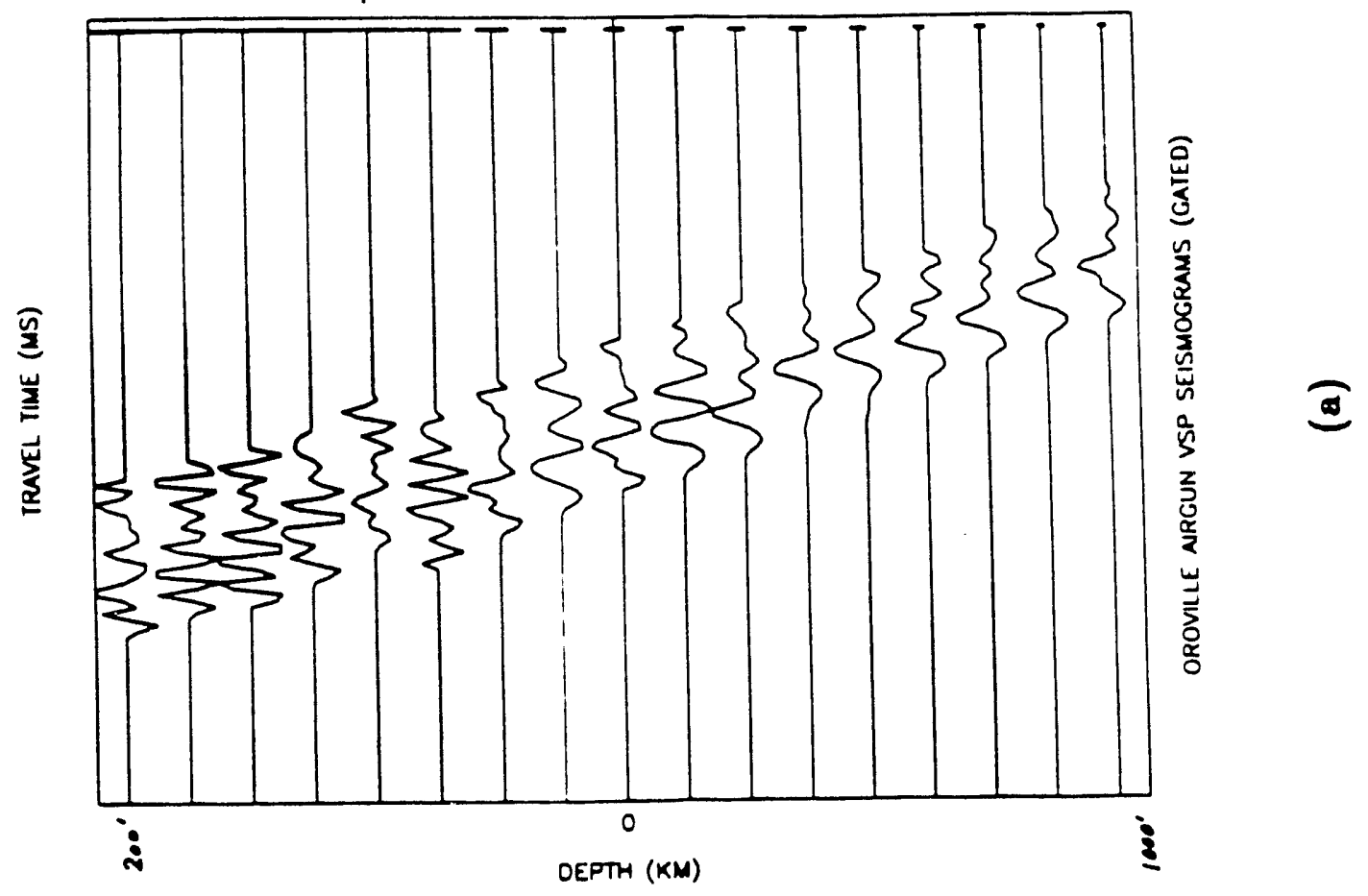




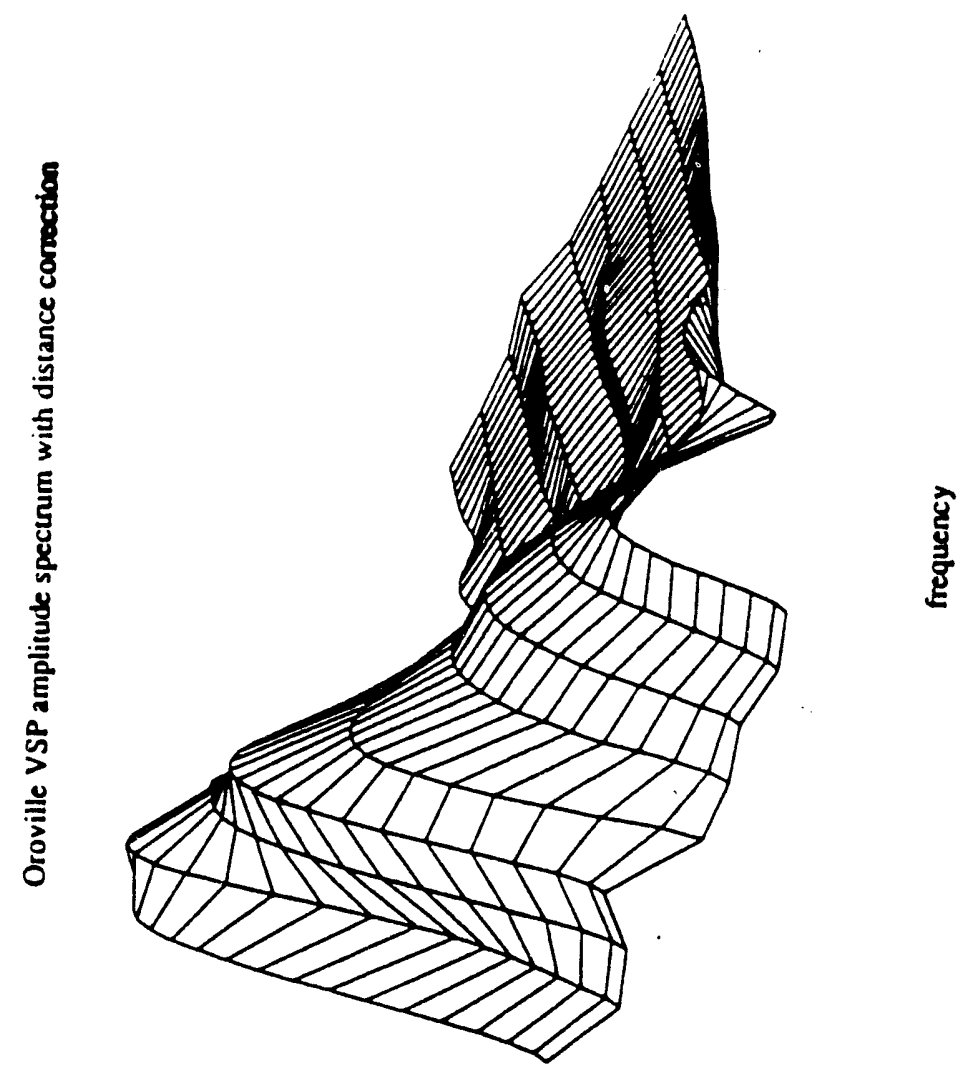

อ

undop

产

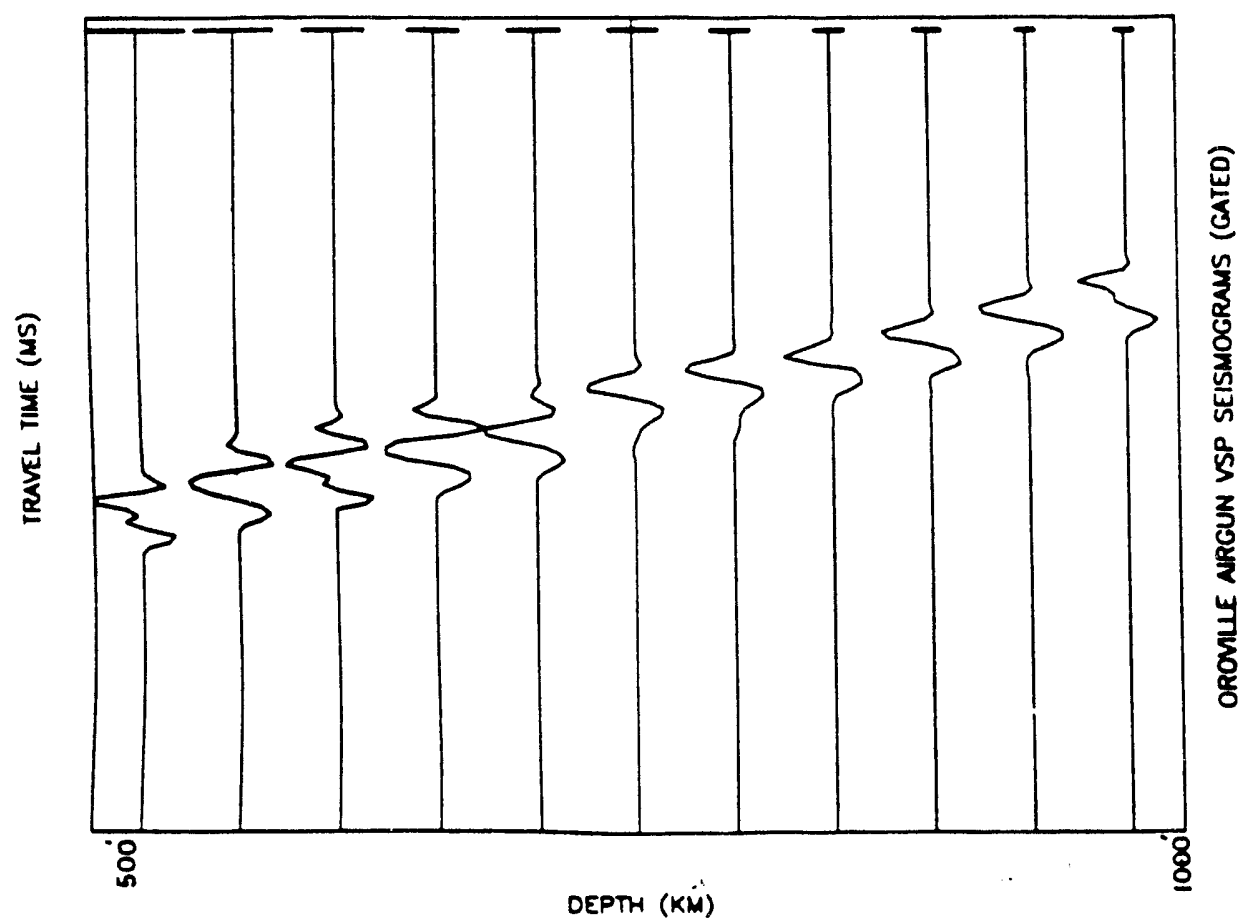

$\Xi$ 


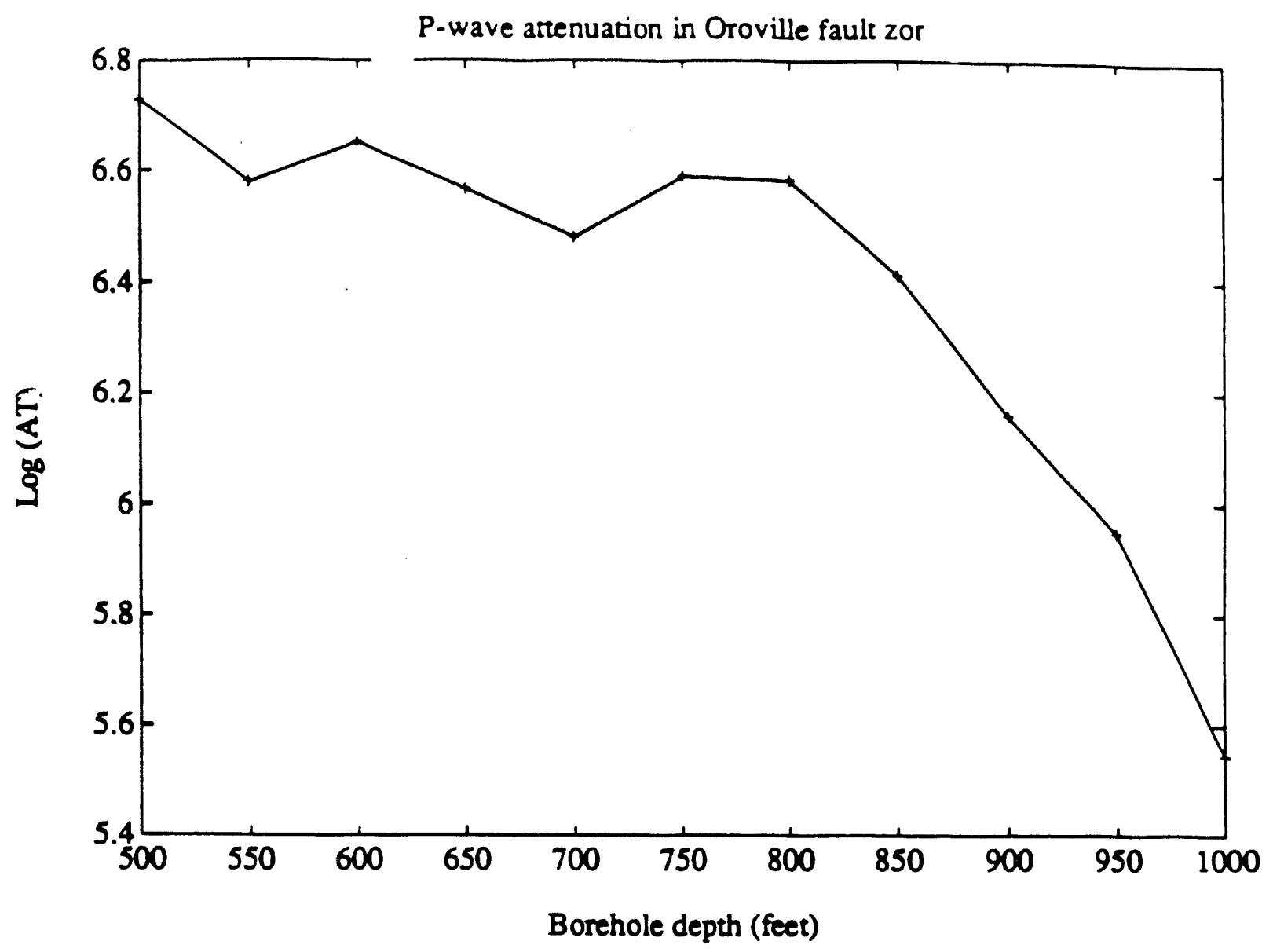

(a)

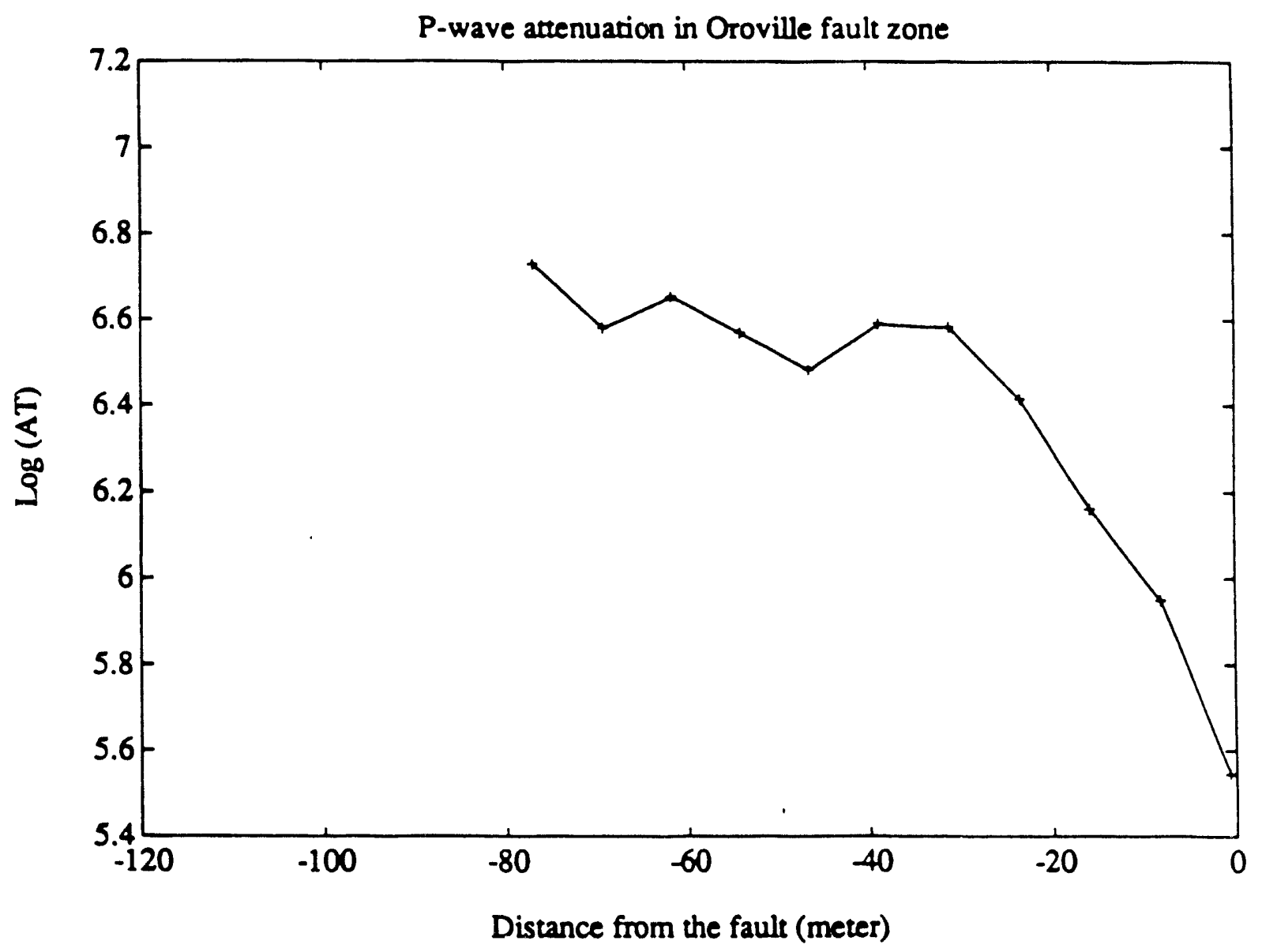

(b)

Fig. 8 
(5) Interpretation of Source Spectra of Some Volcanic Events in Terms of 3-D Masma Body Vibration.

In early 1988 we carried out a field experiment at Kilauea Volcano, supported not by DOE but by NSF and University, during which the volcano was active and generated gas-piston events, long-period events and tremors. Figure 1 shows the recording sites of the data that will be shown in this report. Gas-piston events and tremors were recorded at the site PUO, and long-period events which have their hypocenter under Kilauea summit area, have been recorded at HVO, KEA, ESC, NEN, and few of them at PUO.

Typical examples of energy spectra of a gas-piston event, a tremor and a longperiod event are shown in Figures 2, 3 and 4 respectively. All of them show very narrow peaks and at least some of them are observed repeatedly over many events.

Figure 5 shows the stack of the energy spectra of all components of all stations, except PUO, of a long-period event, and Figure 6 shows the stack of the three components of the same event recorded at PUO. Clearly, the same spectral peaks show up in both figures. Since the propagation path to PUO is longer and quite different (nearly along the East-Rift zone) from those to the other instruments, the agreement between spectral peaks supports the idea that these peaks are not due to a path effect but due to a source effect.

For gas-piston events, all the instruments were located in a very small area and our argument is not as strong as for long-period events, but we observed similar evidence supporting the idea that the source is responsible for the narrow peaks.

The sharp narrowness of spectral peaks of gas-piston events and tremor indicates a Q value as high as 40. Aki et al. (1977) have shown that the damping of the fundamental mode of isotropic oscillation of a liquid sphere embedded in a solid is determined by the impedance contrast between solid and liquid. For high impedance contrast $\mathrm{Q}$ becomes directly proportional to the impedance contrast. Numerical simulations of long-period events (Ferrazzini et al., 1989) by resonance set up in a fluid filled crack also show that $Q$ increases with the impedance contrast and the only way to obtain the high impedance contrast required, is to have bubbles in the magma. Gas seems to be a necessary ingredient 
in the generation of all of those signals in order to explain the sharpness of the spectral peaks as long as the system is linear.

The similarity of the spectral characteristics between gas-piston events and tremor is clear in Figure 7. This figure compares the stacked energy spectra of gas-piston events with a tremor spectrum occurring 10 days before the gas-piston events. In spite of the change of the magmatic activities there is no evidence for a shift in frequency and this stability implies that the system must be stable and linear.

The strongest long-period events recorded have power spectra quite similar to those of gas-piston events and volcanic tremor. They are characterized by a dominant peak at low frequency $(.85 \mathrm{~Hz}$. for the long-period events, around $2.5 \mathrm{~Hz}$ for gas-piston events and volcanic tremor), followed by two or three peaks with decreasing amplitude at frequencies which do not correspond to a simple multiple of the dominant frequency. Neither the spectrum of a resonating fluid-filled pipe (Chouet, 1985) or a spherical magma chamber (Crosson and Bame, 1986) will display such features which seem to be due to a three dimensional effect at the sourc. The 3-D fluid-filled crack model developed by Chouet $(1986,1988)$ and used quite successfully to simulate long-period events observed by Bame and Fehler (1986) during hydrof racture experiments in hot dry rock at Fenton Hill, New Mexico (Ferrazzini et al., 1989) may be more appropriate, but the model does not seem to generate the spectral features of long-period events and gas-piston events mentioned above. The inability may be due to the approximation used in solving the equations of motion and continuity for the fluid in the plane of the crack, i.e. in two dimensions, which may be responsible of the overall strong excitation of a very slow wave analogue to the tube wave, called "crack wave". The excess pressure applied being symmetrical with respect to the crack plane, in the thickness of the crack, only symmetrical modes can exist thus favoring the crack-wave development.

This approximation may be adequate in the case of fluid injection into thin cracks as during hydrofracture experiments but may not be valid in the case of a turbulent three 
dimensional fluid flow that we may expect in a bubbly magma. Chouet's model in its present form does not seem to be able to reproduce the required spectral peaks distribution. Rather than to extend the finite difference scheme of the fluid dynamics from two to three dimensions, a solution which will require an enormous amount of computer time, we decided to consider the excitation of normal modes in a rectangular container, with absorbing walls. This approach occurred to us by noticing that the spectral peak distribution of long period events seems to follow those of the normal modes of vibration of a rectangular room. Let us consider for simplicity a rectangular room with rigid walls of dimensions $L_{x}, L_{y}, L_{z}$. The corresponding eigenfrequencies are given by (Morse and Bolt, 1944):

$$
v=\frac{c}{2} \sqrt{\left(\frac{n_{x}}{L_{x}}\right)^{2}+\left(\frac{n_{y}}{L_{y}}\right)^{2}+\left(\frac{n_{z}}{L_{z}}\right)^{2}}
$$

where the quantities $n_{x}, n_{y}, n_{z}$ are integers greater or equal to zero and each trio of values $\left(\mathrm{n}_{\mathrm{x}}, \mathrm{n}_{\mathrm{y}}, \mathrm{n}_{\mathrm{z}}\right)$ corresponds to a normal mode of the room. In the case of the long-period event shown in Figures 5 and 6 , assuming that the dominant peak at $0.85 \mathrm{~Hz}$ corresponds to the normal mode $(1,0,0)$ and the acoustic velocity equals $1 \mathrm{~km} / \mathrm{s}$, we find the largest dimension of the room on the order of $\mathrm{L}_{\mathrm{x}}=600 \mathrm{~m}$. The second peak occurs at $1.08 \mathrm{~Hz}$, a frequency less than twice the frequency of the first mode, thus this peak may correspond to the normal mode $(0,1,0)$ which leads to the second largest dimension of the room $\mathrm{L}_{\mathrm{y}}=470$ m. The third or fourth peaks may be related to the third dimension of the room if this dimension is not too small compared to $L_{x}$ and $L_{y}$, or may correspond to the normal modes =$(1,1,0)$ or $(2,0,0)$. The eigenfrequencies found for those two modes are respectively 1.37 $\mathrm{Hz}$ and $1.70 \mathrm{~Hz}$ which are very close to the frequencies of $1.34 \mathrm{~Hz}$ and $1.6 \mathrm{~Hz}$ of the observed third and fourth peaks respectively, and suggest that the third dimension of the room $L_{2}$ must be smaller than $L_{x} / 2$. Since a high contrast of impedance between fluid and solid was invoked to explain the sharpness of the observed spectral peaks, it is reasonable 
to find that the spectral peak distribution can be fitted with the resonant frequencies of a fluid-filled box with rigid walls.

Let us now consider the effect of the wall impedance on the resonant frequencies of a room. Standing waves in rooms have been the subject of numerous studies, in room acoustics when the wavelength of the sound wave is much shorter than the dimension of the room, a geometrical ray analysis is generally more fruitful. In our case, only the lowest modes of vibration seem to be excited in the observation and the normal mode analysis should be a more efficient method to obtain the acoustical response of the room and to determine to what extent the resonant rectangular room may be compared to the finite difference 3-D fluid-filled crack model.

Morse and Bolt (1944) and Morse and Ingard (1968) gave an analytic solution for the Green function in various cases of rectangular rooms and the approximate solution for irregularly shaped rooms. All these solutions are based on the expansion of the Green function in series of normal modes, and this is the approach that we will follow
compute the normal velocity field at the surface of the cavity which may be compared
directly with the finite difference solution. For this purpose, we need first to get the
eigenvalue and eigenfunction solutions for the standing waves in the rol

Let us consider a rectangular fluid-filled room with dimensions given by $\mathrm{L}_{\mathbf{x}}, \mathrm{L}_{\mathbf{y}}$, $\mathrm{L}_{\mathrm{z}}$, let $\mathrm{V}$ be the volume of the room, $\mathrm{S}$ the surface enclosing this volume. The fluid velocity and pressure may be expressed in terms of the velocity potential $\psi$ :

$$
\begin{aligned}
& \vec{V}=-\vec{\nabla} \psi \\
& p=\rho_{\frac{\partial}{\partial t}} \frac{\partial t}{\partial t}
\end{aligned}
$$

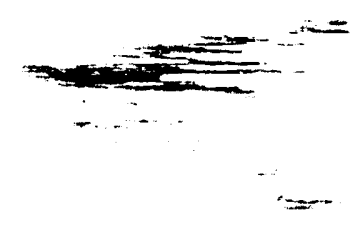

and $\psi$ satisfies the equation:

$$
\nabla^{2} \psi=-K^{2} \psi
$$

Taking the coordinate system at the center of the room, agenteral solutur tor $\psi$ may be writien: 


$$
\psi_{n}=\left\{\begin{array}{l}
\cos \\
\sin
\end{array}\left(\frac{\pi q_{n_{x} x}}{L_{x}}\right), \begin{array}{l}
\cos \\
\sin
\end{array}\left(\frac{\pi q_{n_{y} y}}{L_{y}}\right), \begin{array}{l}
\cos \\
\sin
\end{array}\left(\frac{\pi q_{n_{z} z}}{L_{z}}\right)\right.
$$

Where cos and sin holds for even and odd modes with respect to the subscript value of $n_{i} ; i=x, y, z$ which refer to the $n_{i}^{\text {th }}$ mode along the $i$ axis and the subscript $n$ of $\psi$ refers to a trio of values $\left(\mathrm{n}_{\mathrm{x}}, \mathrm{n}_{\mathbf{y}}, \mathrm{n}_{\mathbf{z}}\right)$.

The characteristic values $q_{n}$ are in general complex numbers and may be expressed by the form $q_{n i}= \pm \mu$ - iv where $\mu$ is the real part corresponding to the wavenumber and $v$ is the imaginary part representing the attenuation. These two parameters are determined by the boundary condition that the ratio of pressure to normal velocity at each wall equals the impedance $\mathrm{Z}$ corresponding to outgoing waves in the medium immediately outside the room:

$$
\mathrm{Z}=\overline{\mathrm{V}} \rho_{\mathrm{f}} \frac{\partial \psi}{\partial \mathrm{t}} / \frac{\partial \psi}{\partial \mathrm{n}}
$$

where $\mathrm{n}$ is the normal to $\mathrm{S}$ oriented outward the room.

Fixing the boundary condition at $x= \pm \sum_{x} / 2$ and writing $\beta=\rho_{f} c / Z$, we get the equation for $q_{n_{x}}$ :

$$
\frac{\pi \mathrm{q}_{\mathrm{n}_{\mathrm{x}}}}{2} \operatorname{tg}\left(\frac{\pi \mathrm{q}_{\mathrm{n}_{\mathrm{x}}}}{2}\right)=-\mathrm{ikL} \mathrm{L}_{\mathrm{x}} \beta
$$

for the even modes, and

$$
\frac{\pi q_{n_{x}}}{2} \operatorname{cotg}\left(\pi q_{\left.n_{x} / 2\right)}=+i k I_{x} \beta\right.
$$

for the odd modes.

The same boundary condition applied at $\mathrm{y}= \pm \mathrm{L}_{\mathrm{y}} / 2$ and $\mathrm{z}= \pm \mathrm{L}_{\mathrm{z}} / 2$ yields similar equations for $q_{n y}$ and $q_{n_{z}}$. In these equations, if $\beta$ does not depend on the angle of incidence of the sound wave then the quantities $a_{n_{x}}$ depends only on properties of the $x$ walls and the calculations can be carried out independently for each pair of walls and the results combined at the end. 
Dropping the subscript for $q$ and $L$ and putting $q=\mu$-iv and $\beta=\xi$-i $\sigma$, the separation of the preceding equations into real and imaginary part gives for the even modes:

$$
\begin{aligned}
& \left(v \tanh \frac{\pi v}{2}\left[1+\tan ^{2} \frac{\pi \mu}{2}\right]-\left(\mu \tan \frac{\pi \mu}{2}\left[1-\tanh ^{2} \frac{\pi \nu}{2}\right]\right) / 2\left(1+\tanh ^{2} \frac{2 \pi \nu}{2} \tan ^{2} \frac{\pi \mu}{2}\right)=\frac{k L}{2 \pi} \sigma\right. \\
& \left(v \tan \frac{\pi \mu}{2}\left[1-\tanh \frac{2 \pi \nu}{2}\right]+\left(\mu \tanh \frac{\pi \nu}{2}\left[1+\tan \frac{\pi \mu}{2}\right]\right) / 2\left(1+\tanh ^{2} \frac{\pi \nu}{2} \tan \frac{2 \pi \mu}{2}\right)=\frac{k L}{2 \pi} \xi\right.
\end{aligned}
$$

and for the odd modes:

$$
\begin{aligned}
& \left(v \tanh \frac{\pi v}{2}\left[1+\tan ^{2} \frac{\pi \mu}{2}\right]+\left(\mu \tan \frac{\pi \mu}{2}\left[1-\tanh \frac{2 \pi \nu}{2}\right]\right) / 2\left(\tanh \frac{2 \pi \nu}{2}+\tan ^{2} \frac{\pi \mu}{2}\right)=\frac{\mathrm{kL}}{2 \pi} \sigma\right. \\
& \left(-v \tan \frac{\pi \mu}{2}\left[1-\tanh ^{2 \pi \nu} \frac{2 \pi}{2}\right]+\left(\mu \tanh \frac{\pi v}{2}\left[1+\tan ^{2} \frac{\pi \mu}{2}\right]\right) / 2\left(\tanh ^{2} \frac{\pi \nu}{2}+\tan ^{2} \frac{\pi \mu}{2}\right)=\frac{\mathrm{kL} \xi}{2 \pi}\right.
\end{aligned}
$$

Each pair of equations can be solved numerically for $\mu$ and $v$ using Newton-Raphson's method, given the values of $L, \xi$ and $\sigma$, and of the frequency $\omega / 2 \pi=k c / 2 \pi$. Assuming that the impedance contrast does not depend on the angle of incidence of the sound wave, if acceptable in the case of a hard walled room, may not be accurate to compute the pressure and velocity fields at the surface of the room. For given dimensions of the room and a mode of vibration, the angles of incidence of the sound wave can be found and the impedance $Z$ at each wall surface can be determined. The boundary equations for the six walls can then be solved simultaneously. We are currently working on this problem. 


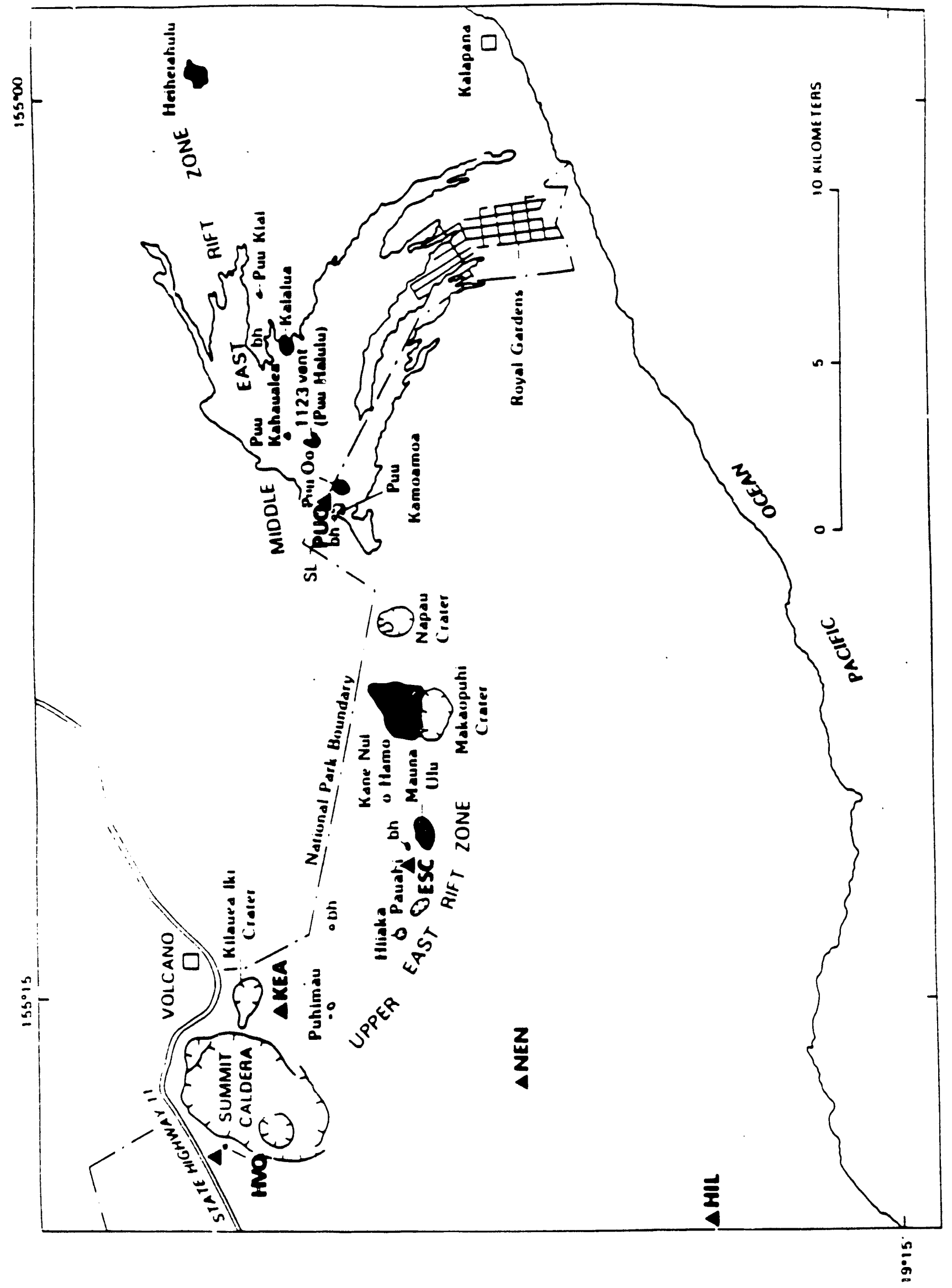


i

ف묘

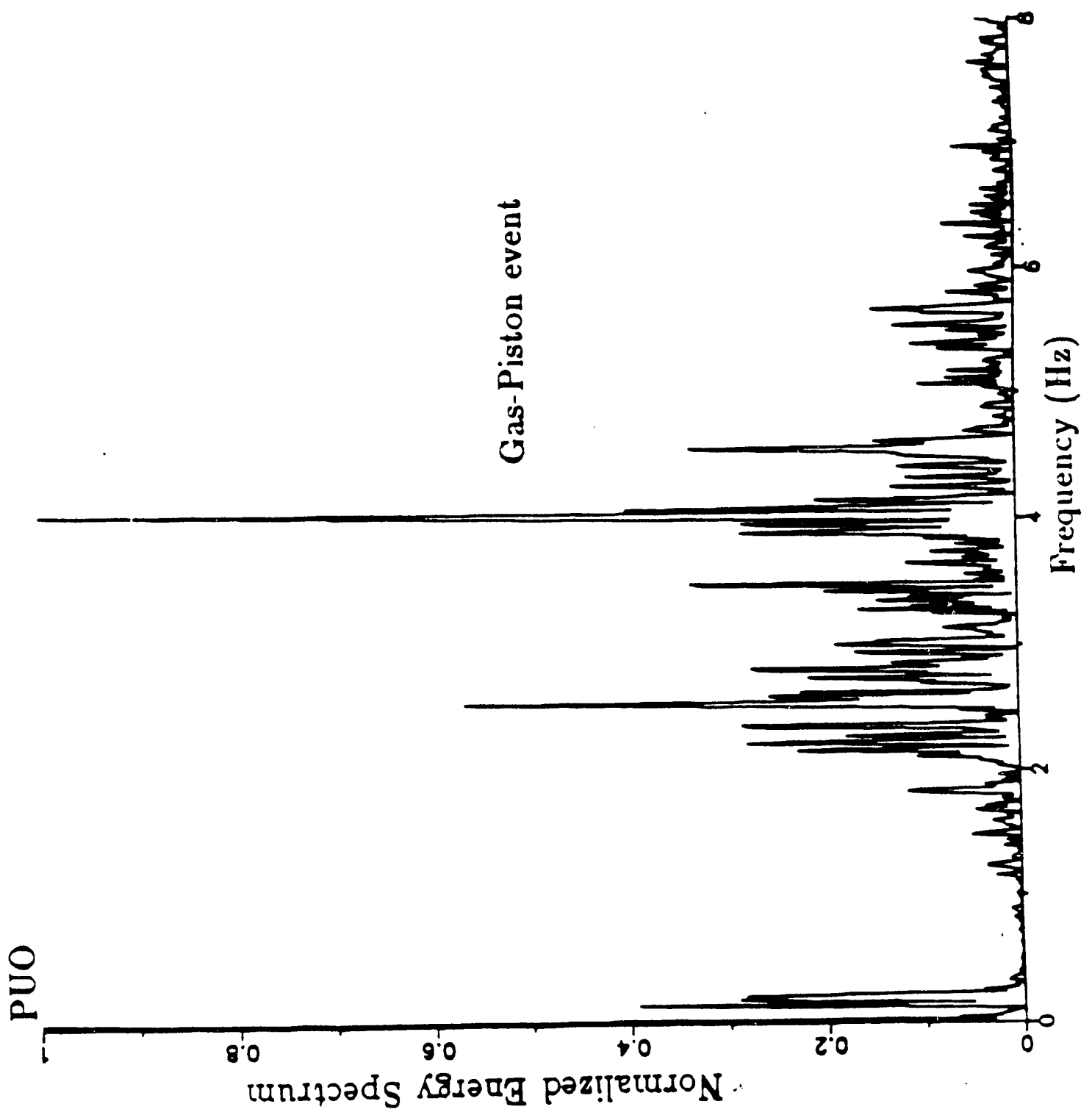




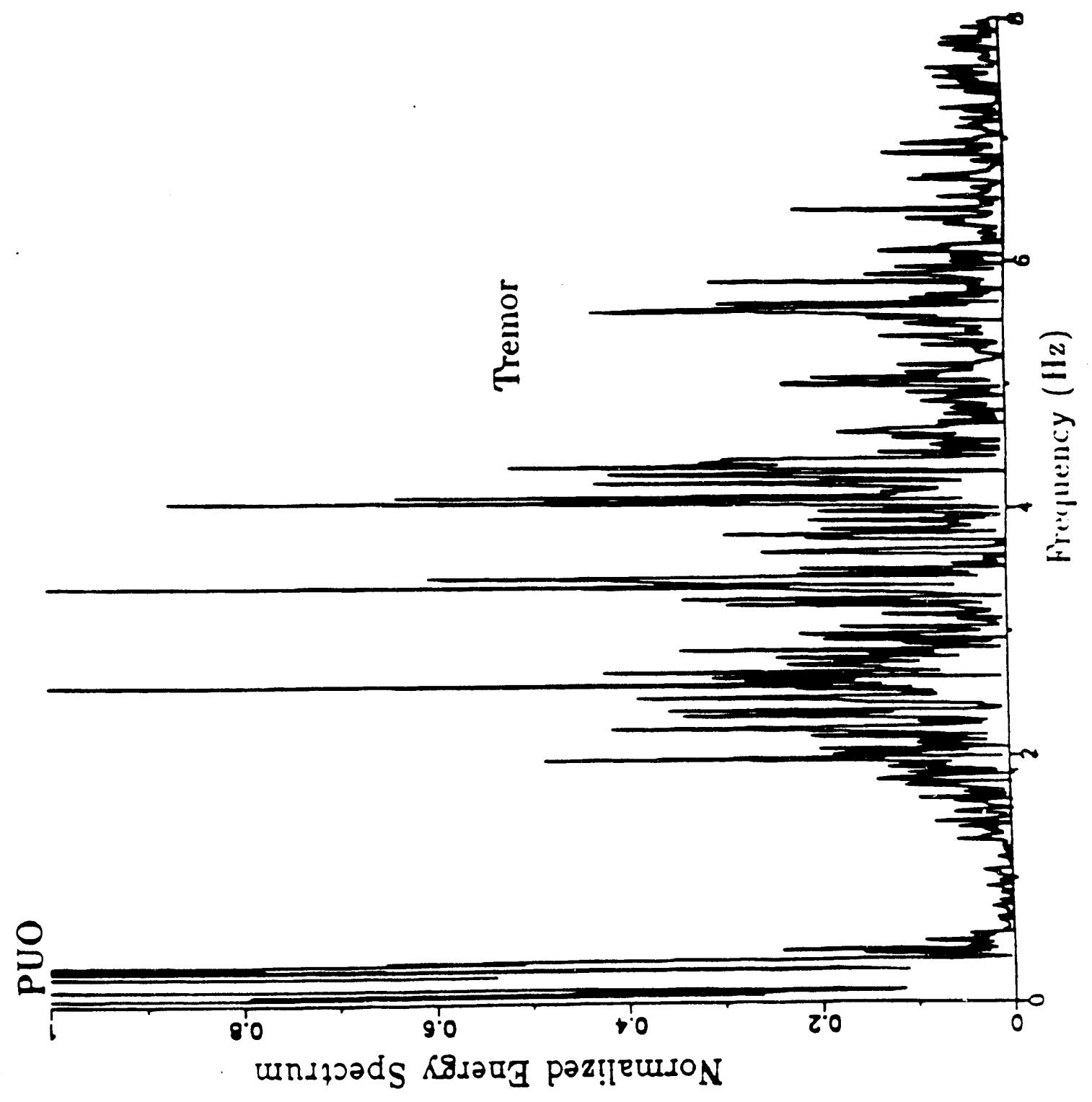




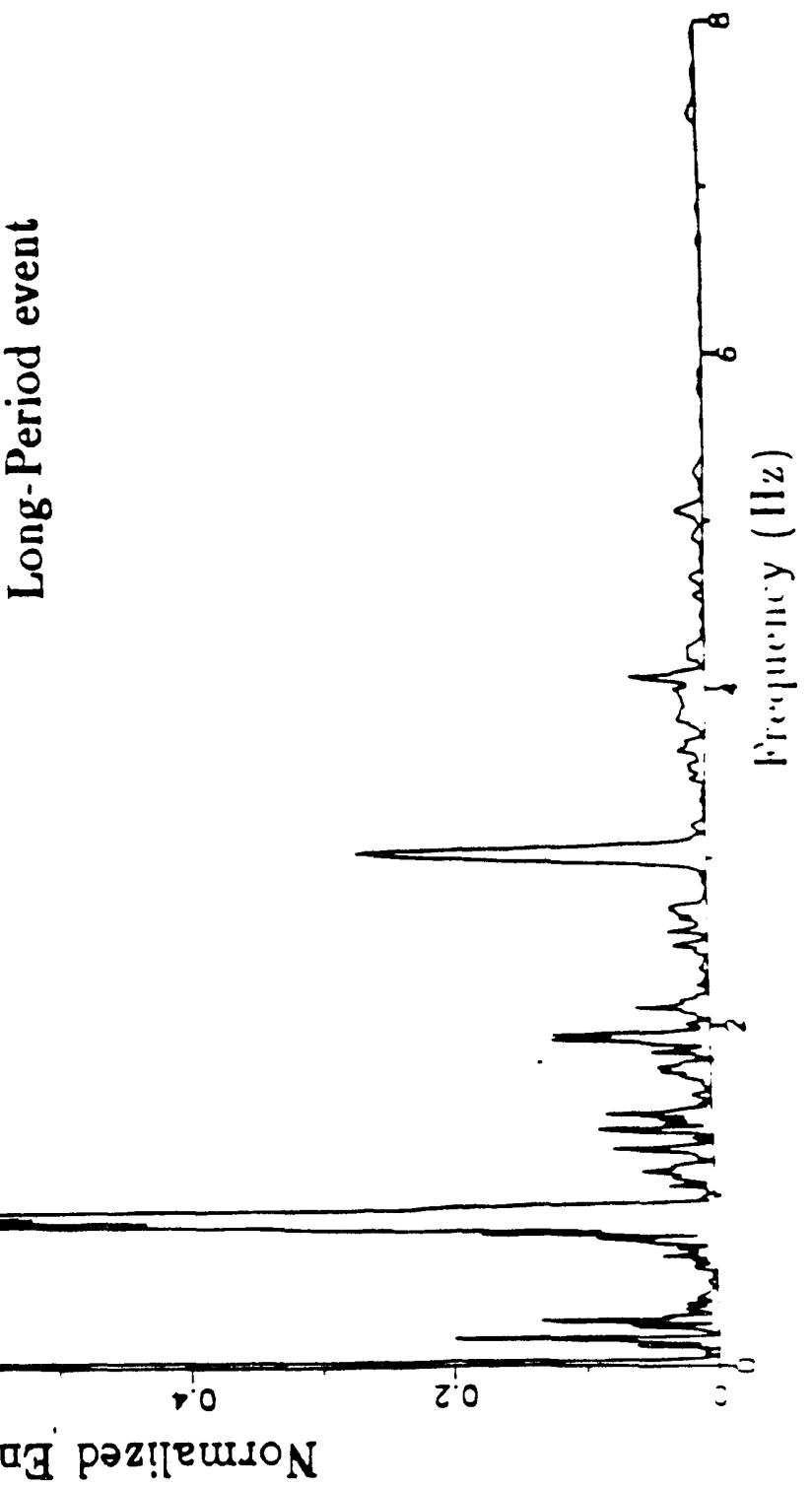




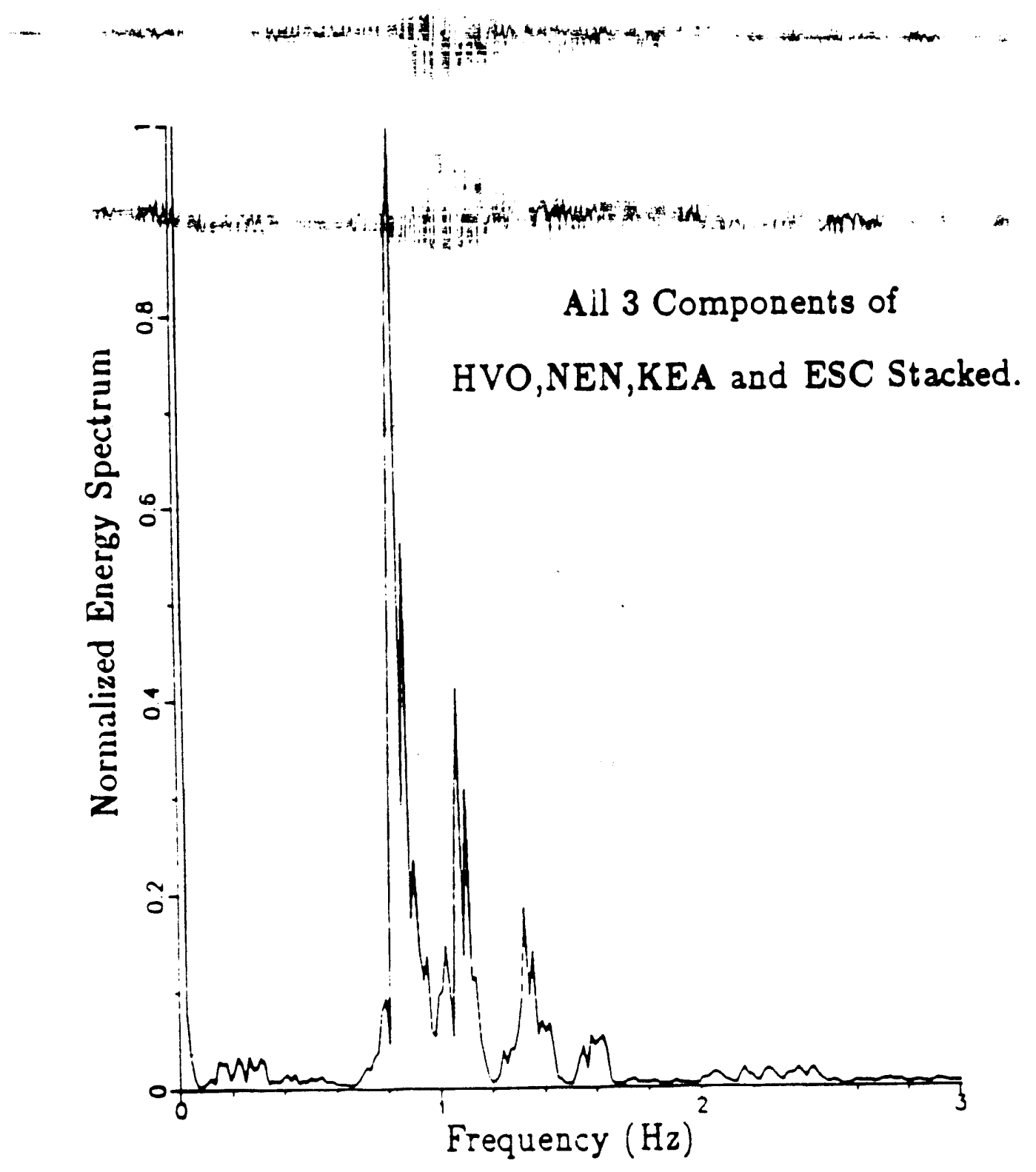

Fig. 5. 


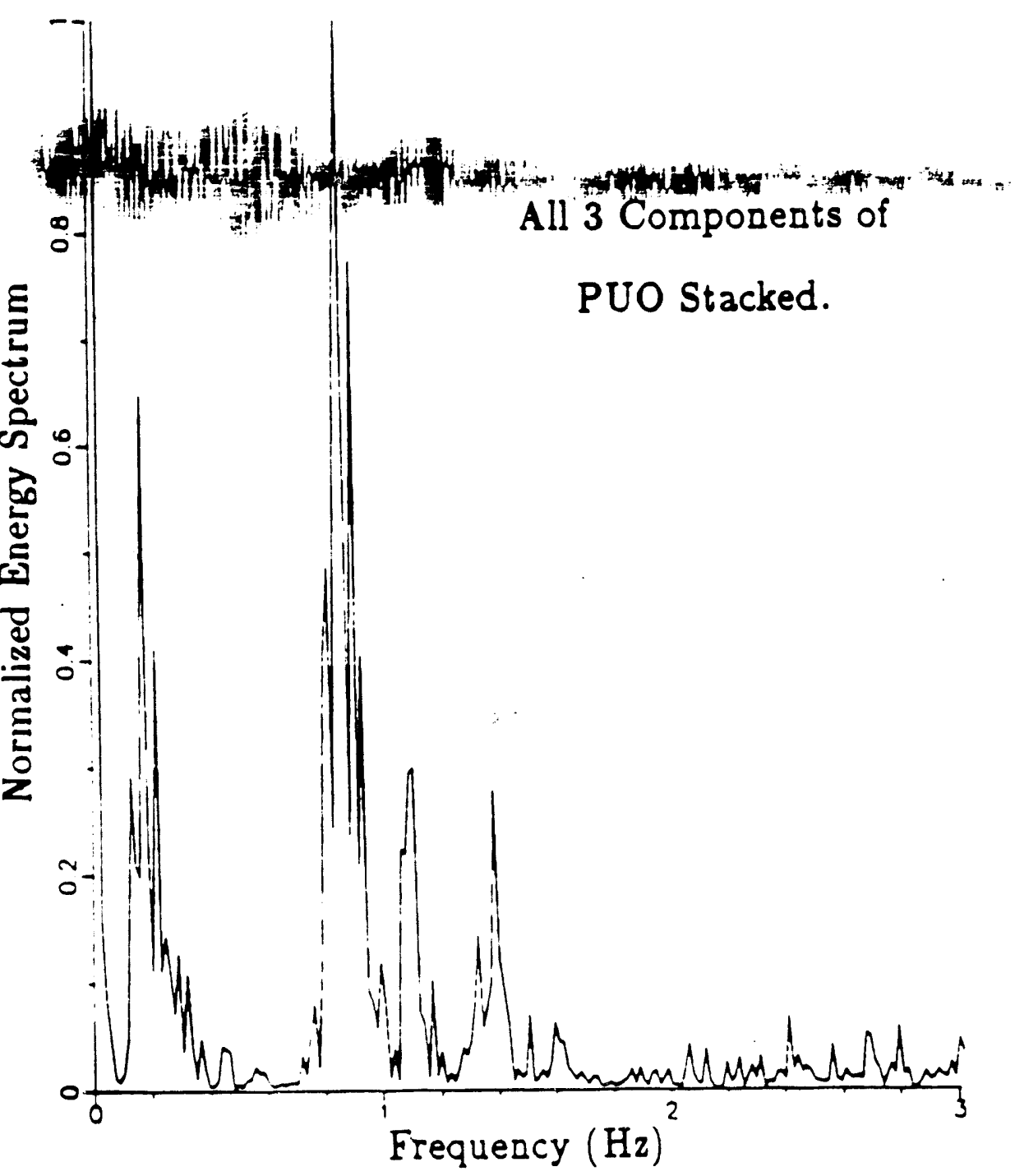

Fig. 6. 


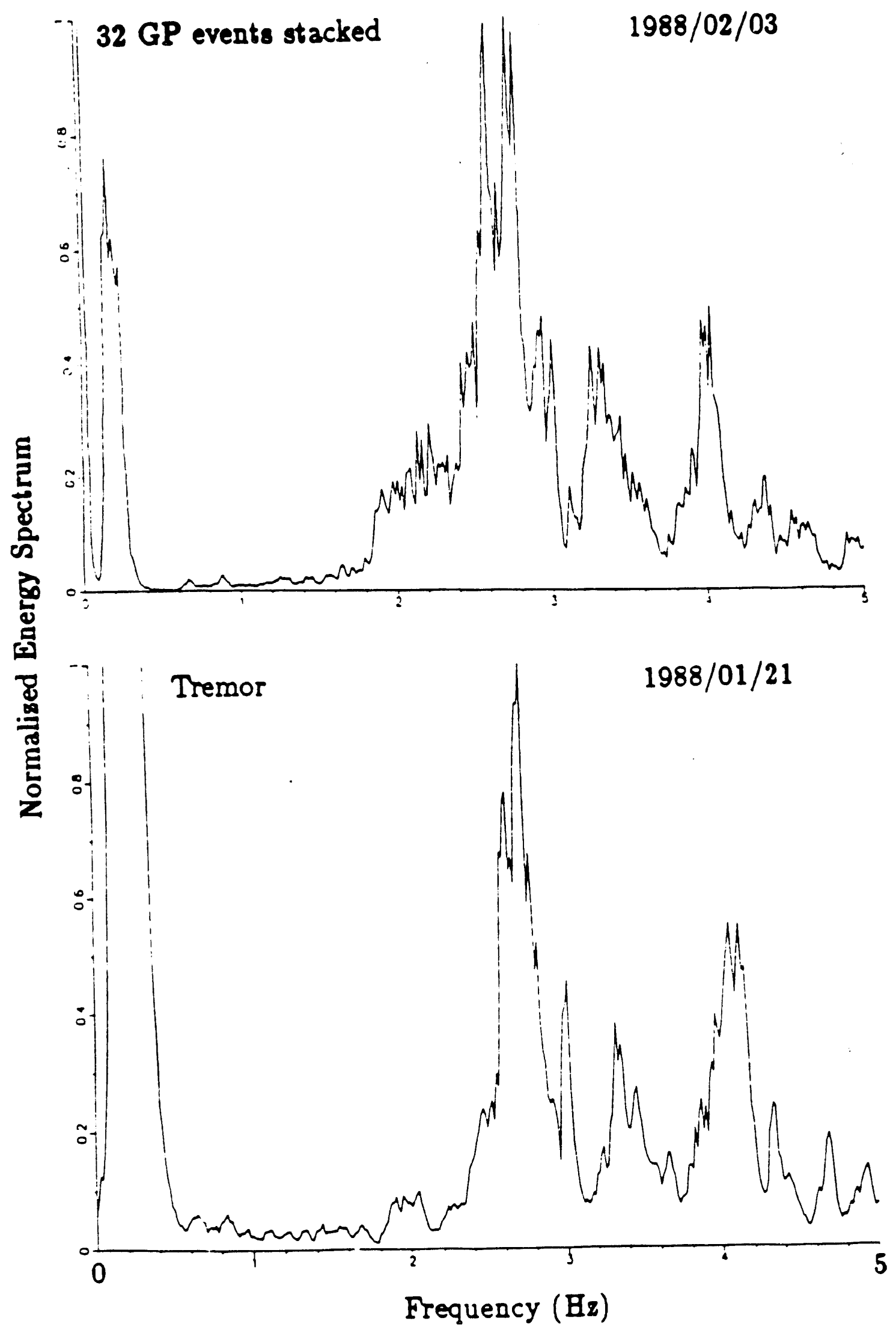

Fig.7. 
Testing of fiber optical borehole seismometer.

A prototype fiber optic seismograph reported in last year's progress report has been continuously operated in a shallow instrument vault housing a 3-component station of the USC Los Angeles Basin seismic network. The performance of our fiber optic seismograph has been satisfactory in both vertical and horizontal mode as shown in Figures 1 and 2 which compare time records of local earthquakes obtained by the fiber optic seismograph and standard network seismograph (LAC). Figure 3 also shows a satisfactory agreement in the frequency domain. Several operational details remain to be tested and/or developed. At present the sensitivity of the fiber optical sensor is an order of magnitude less than that of the L4C seismometer. The sensitivity of the fiber optic sensor can be improved by upgrading the quantity of the light source and photo detector, and by increasing the inertial mass. Increasing the inertial mass has the further benefit of broadening the low frequency side of the instrumental passband. The prospects for a horizontal borehole seismic sensor with frequency response at or possibly well below $1 \mathrm{~Hz}$ are appealing for deep borehole studies of natural seismicity.

Several aspects of the performance of the fiber optic sensor are not presently thoroughly studied. The prototype sensor is more fragile than a seismometer. A sharp blow that does not damage a seismometer may break the fiber. Still at question is whether the fiber is significantly weakened due to steady state abrasions of the fiber by the teeth of the deforming mass or whether the fiber is simply overdriven by deflection of the mass. We have postponed placing the prototype at a significant depth in a borehole until we understand the extent of fiber abrasion. As a measure or protection against abrasion we will coat the deforming jaws with a layer of aluminum. For protection against the hazards to lowering the sensor downhole, we can install a motor driven shim that pins the inertial mass for deployment, than retracts for operation.

A drawback to the fiber optic accelerometer detection of vertical motion is that when the pendulum system is horizontal the inertial mass creeps toward or away from the optical 
fiber. Thus, there is a steady baseline shift in the sensor. Since inertial and gravitational masses are indistinguishable, there may be no passive mechanical configuration which overcomes the slow deformation of the accelerometer mass. It may be possible to stabilize the creep with a stiff fiber, but mindful of fiber fabrication we have not attempted this solution.

We conclude that detection of horizontal or vertical acceleration in the short period seismic passband 5 to $30 \mathrm{~Hz}$ via distortions of an optical fiber compares well with a standard short period seismometer and a force-balanced accelerometer. At feasible sensitivities, the fiber optic sensor can probably outperform available electromechanical horizontal motion sensors available for deployment in the confined space and high temperatures of deep crustal boreholes or geothermal wells. 

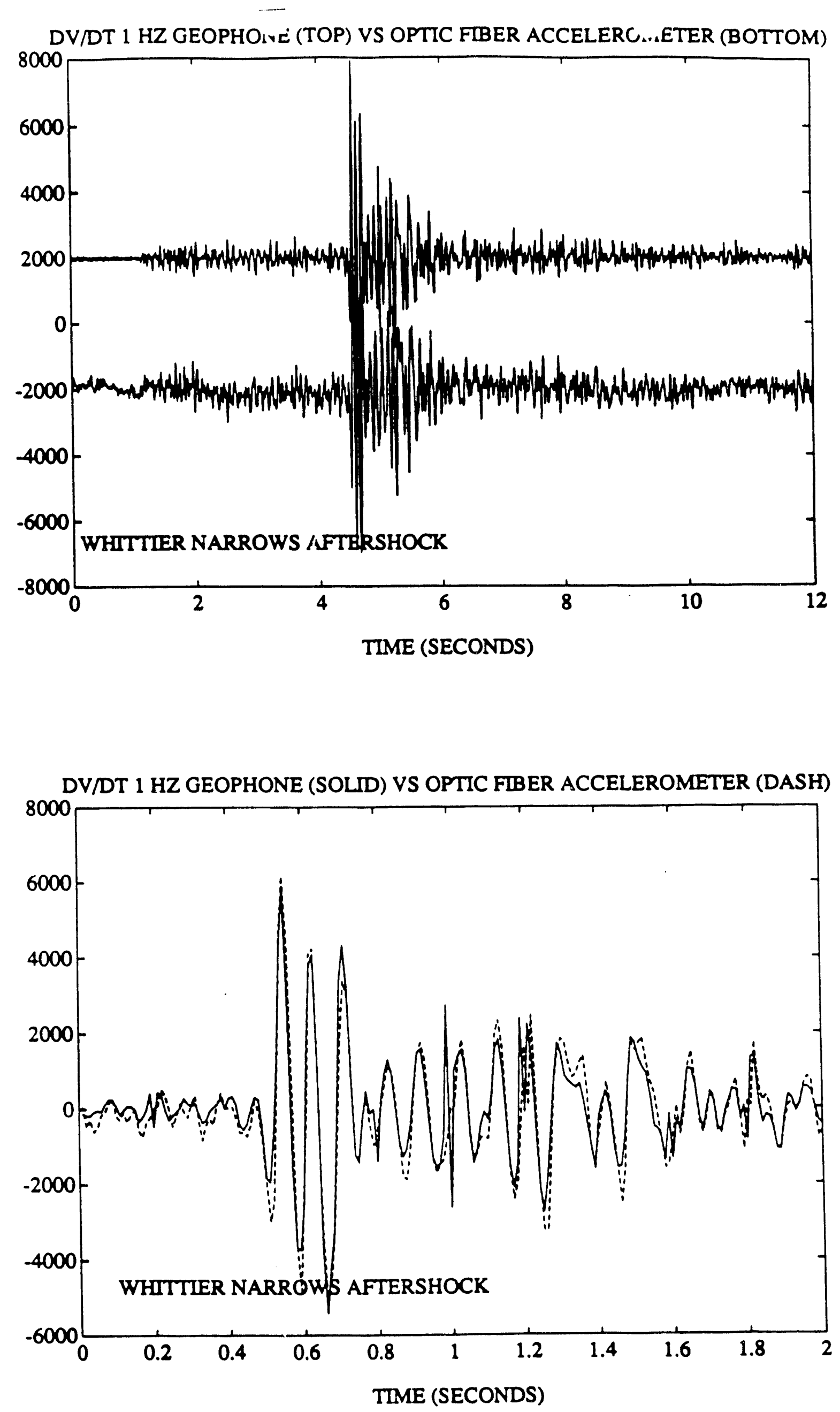

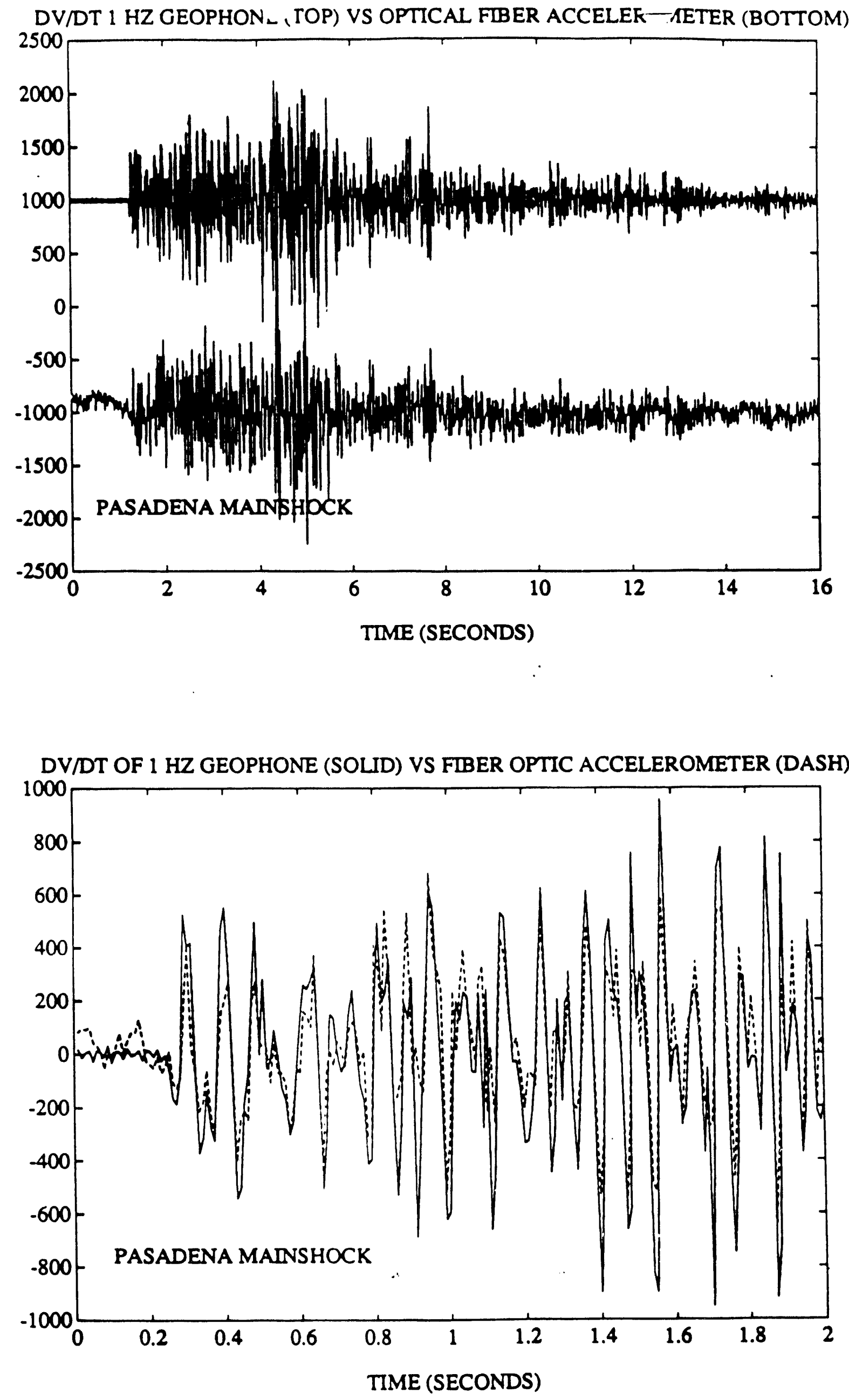


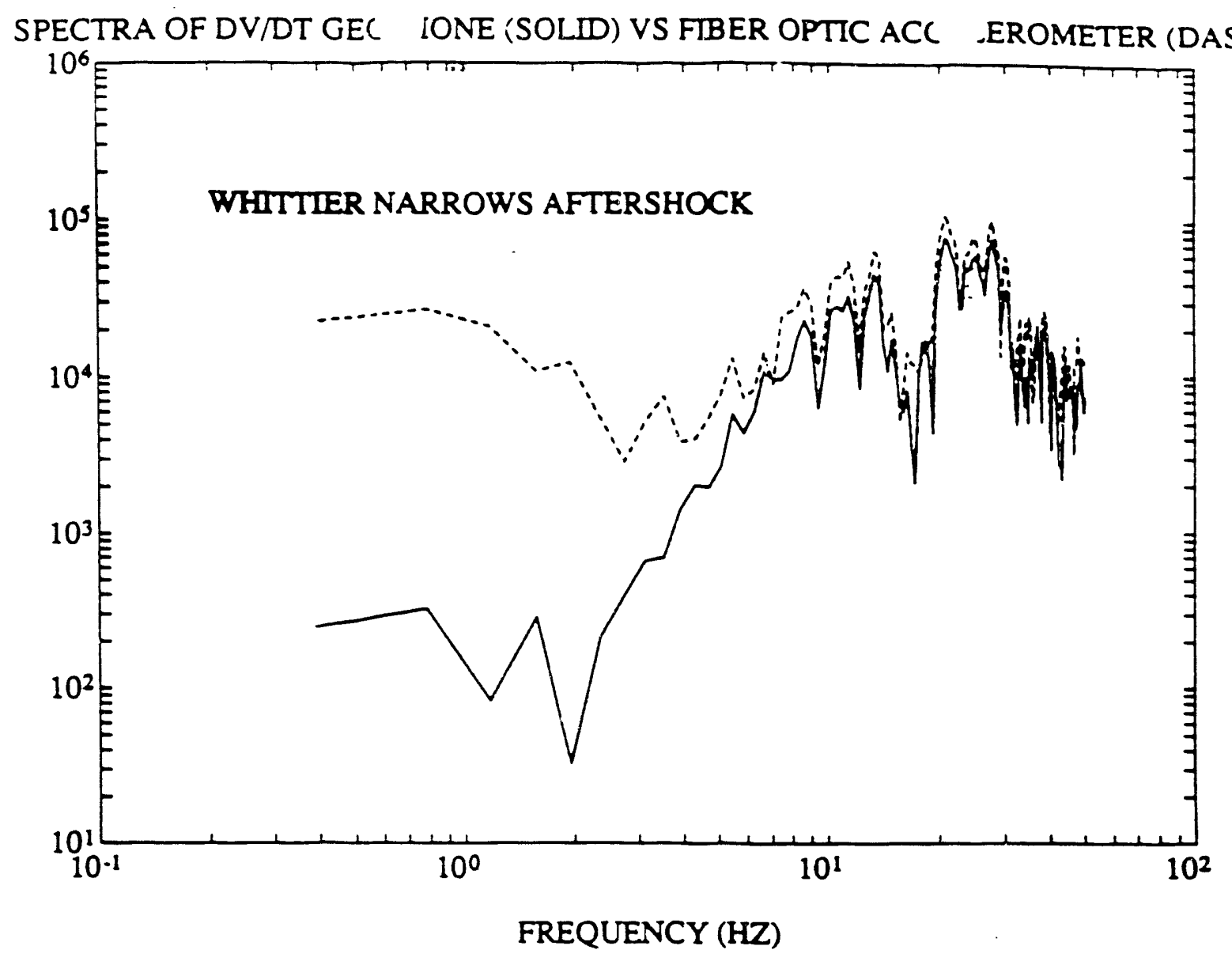

SPECTRA GURALP ACCELEROMETER(SOLID) VS FIBER OPTIC ACCELEROMETER(DASH)

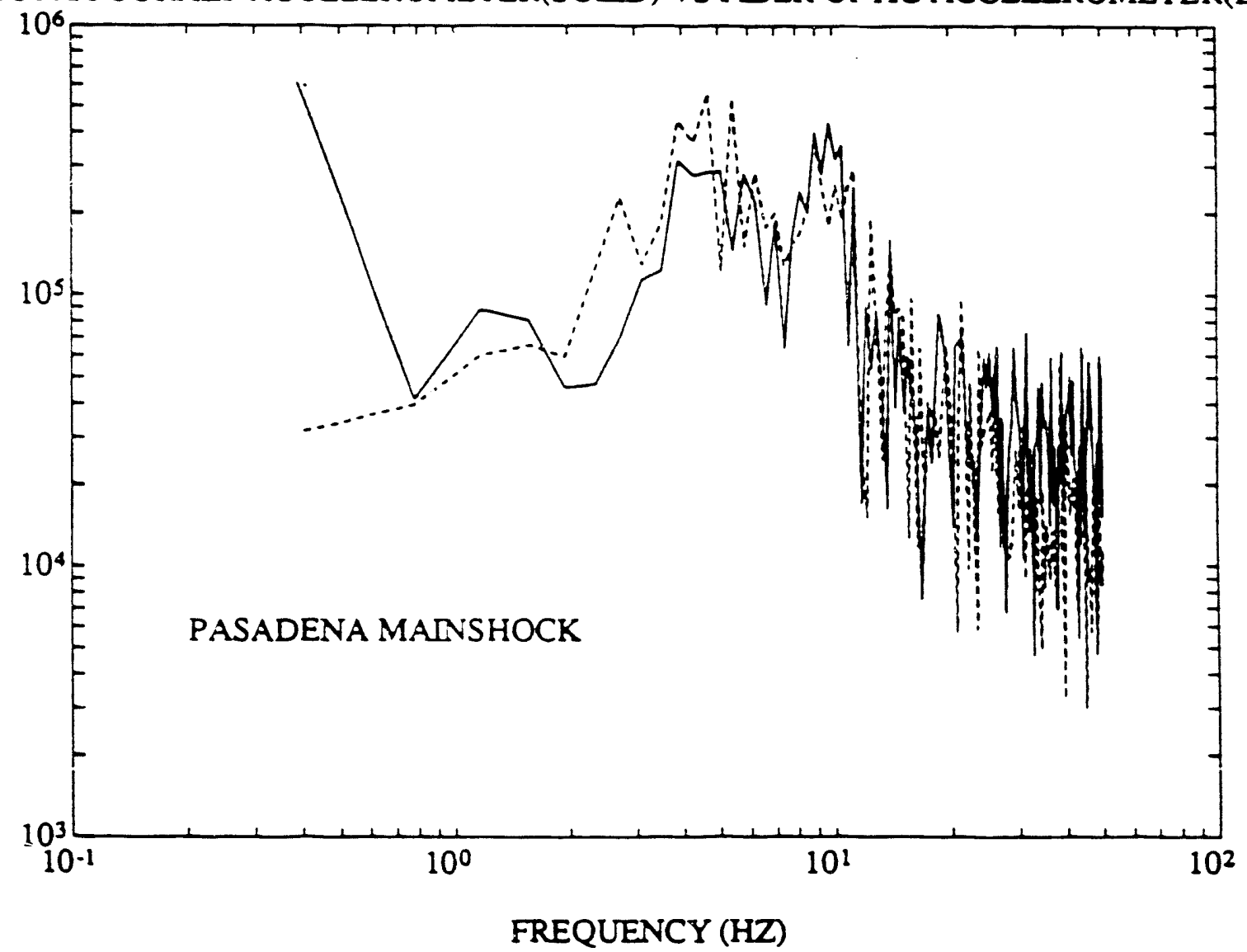




\section{PROPOSED RESEARCH FOR THE NEXT YEAR.}

As described in the preceding progress report for the second year of our 5-year project, we have made a considerable progress and gained momentum in all aspects of originally proposed task. In the next one-year period, we shall continue along the lines of research developed in the last two years.

First, we shall complete one or more papers on the seismic $Q$ and velocity structure of the Valles Caldera, using the results of Robert's thesis work to be submitted to appropriate journals. We plan to do some additional works before completing these papers, because the extremely low $Q$ value we found has triggered vigorous discussions among the members of thesis committee, and ve want to analyze the existing data available at the Los Alamos National Laboratory to further substantiate our conclusion. This work will be done by the P.I. and Peter Roberts who is now working at the LANL as a postdoctoral fellow.

Secondly, we shall investigate systematically the applicability of so called "Born inversion" to the seismic waveform data using the models of high-velocity and low-velocity inclusion for various velocity contrasts. The question of the validity of linearized iterative inversion procedure involved in the Born inversion is fundamental to the inverse problem in seismology, and our study will give an insight to this problem by the use of our new synthetic seismogram method, namely, the Gaussian beam-boundary integral method described in the progress report. This study will be carried out by Ratael Benites as a part of his Ph.D. thesis work, which is expected to be completed in the next year. Thirdly, we plan to improve our interpretation method for fracture related seismic attenuation in VSP data. In the past, our method enabled us to estimate the orientation and density of fractures, but not the sizes and shapes of fractures, because our method was based on Hudson's (1981) formula of elastic constants for aligned cracks which are valid only for wavelengths much longer than the crack size. We need formulas valid for wavelengths 
comparable to a shorter than the crack size, to make a reliable estimation of the size and shape of the crack.

We made a literature search, but failed to find such formulas. The existing theories applicable to wavelengths comparable to and shorter than the scale length of inhomogeneity are mostly concerned with an elastic medium perturbed randomly but continuously according to an autocorrelation specified by the correlation distance and mean squared fluctuation.

We propose to study the intrinsic as well as scattering attenuation due to cracks for a wide range of wavelength relative to the crack size, following the $\mathrm{T}$-matrix method as used by Varadan et al. (1979) and Bring et al. (1982) for electromagnetic waves and by Varadan et al. (1983) for acoustic waves. They characterize the random medium by an effective complex wave number $\mathrm{k}$ which is a function of the concentration, size and distribution of scatterers. We shall extend their method to the problem of elastic waves in composite media including the medium containing fluid-filled cracks. This work will be carried out by Yongong $\mathrm{Li}$ who is now a post-doctoral fellow at USC.

Fourth, we shall continue to work on the study of source spectra of volcanic events, including the gas-piston and long-period events and tremors observed in the Kilauea volcano, using the model of 3-dimensional chamber filled with magma and gas. We shall solve the equations described in item (5) of the progress report and compare the solution with those obtained earlier by the finite difference calculation under the assumption of thin crack. We II then apply our new solution to various observations at Kilauea in order to determine the structure and material property of magma system. The new interpretation method will be useful for studying seismic signals generated in continental geothermal sites, because we found at the recent Capri workshop on volcanic seismology that many of the features of seismic signals are common between the Hawaiian volcano and volcanoes in Japan and Italy. 
Finally, we shall continue the testing of fiber optic sensor in boreholes at USC seismic network sites. Lab tests and surface deployment tests will be conducted on a higher mass, lower stiffness version of the sensor (in order to improve its sensitivity and low frequency response). A second horizontal component and a vertical component will also be constructed in-house and packaged for borehole deployment. The borehole packaging will include devices for shimming the deformer jaws for downhole and uphole transport. The 3-component package will be monitored via the USC Los Angeles Basin seismic network communications and recording facility (as done presently).

The Magma Energy Extraction Project deep drill hole at the Long Valley Caldera, $\mathrm{CA}$, is scheduled to begin its Phase I drilling in July of this year. Phase I calls for a cased 2500 ' hole through surface rhyolites and tuffs into the Bishop Tuff formation. The Bishop Tuff, which originated in the last major eruptive episode at the Long Valley Caldera, is thought to be a critical formation for fluid circulation, reservoir recharge and heat extraction. As such it represents an important target for 3-component VSP profiling for fracture related anisotropy.

We will conduct a reconnaissance VSP survey at the end of Phase I drilling. The level of effort will be comparable to a scaled up Hi Vista VSP reported on above. The present upgraded USC VSP digital tool capability allows us to employ a vibrator source for adequate penetration and offset. The multi-offset, multi-azimuth reconnaissance survey will establish basic VSP parameters: P-wave penetration through lossy uppermost rhyolite and tuff layers; the degree of $\mathrm{P}>\mathrm{S}$ conversion at the Bishop Tuff interface; the P-and Svelocity structure; the dip of interface; and the presence or absence of horizontal reflectors in the tuff (and possibly below the tuff). The horizontal component data will be analyzed for the extent of and direction of shear wave splituing. The vertical component data will be analyzed for reflectivity below the hole.

The major VSP cost will be the vibrator. At about $\$ 1000 /$ day plus transport for tis vibrator, a 3 day shoot with one day in hand for set up and repeat runs, will cost about 
$\$ 5,000$. Three full vibrator days will yield near-offset 10 meter interval velocity and reflectivity data, and four to six multi-azimuth intermediate offset, runs for shear wave data. We will use part of our field expense funding for vibrator rental; USC has agreed to provide additional funds to make up the difference.

In the event that the hole is too hot for our tool, we will use our high temperature analog tool with downhole buffering electronics. The analog option is the less desirable as the analog tool clamping is less firm than that of the digital tool, and the analog tool does not provide independent tilt orienting information. However, analog tools are the industry standard and we can complete the experiment with it. 


\section{REFERENCES}

Aki, K. and K. L. Lamer (1970). Surface motion of a layered medium having an irregular interface due to incident plane SH waves, J. Geophys. Res. 75:933-954.

Aki, K., B. Chouet, M. Fehler, G. Zandt, R. Koyanagi, J. Colp, R. Hay (1978). Seismic properties of a shallow magma reservoir in Kilauea Iki by active and passive experiments, J. Geophys. Res. 83:2273-2282.

Aki, K., M. Fehler and S. Das (1977). Source mechanism of volcanic tremor: Fluiddriven crack models and their application to the 1963 Kilauea eruption, J. Volcanol. Geotherm. Res. 2, 259-287.

Bame, D. and M. Fehler (1986). Observations of long-period earthquakes accompanying hydraulic fracturing, Geophys. Res. Lett. 13, 144-152.

Bring, V:N., V. V. Varadan and V. K. Varadan (1982). Coherent wave attenuation by a random distribution of particles, Radio Sciences, v. 17, no. 5, 946-952.

Brown, L. D., C. E. Chapin, A. R. Sanford, S. Kaufman, J. Oliver (1980). Deep structure of the Rio Grande rift from seismic reflection profiling, J. Geophys. Res. $85: 4773-4800$.

Cerveny, V. (1972): Seismic rays and ray intensities in inhomogeneous anisotropic media, Z. Geophysik, 38, 565-577.

Chouet, B. (1988). Resonance of a fluid-driven crack: radiation properties and implications for the source of long-period events and harmonic tremor, J. Geophys. Res. 93, 4375-4400.

Chouet, B. (1986). Dynamics of a fluid driven crack in three dimensions by the finite difference method, J. Geophys. Res. 91, 13,967-13,992.

Chouet, B. (1985). Excitation of a buried magmatic pipe: A seismic source model for volcanic tremor, J. Geophys. Res. 90, 1881-1893.

Chouet, B., R. Y. Koyanagi, K. Aki (1987). Origin of volcanic tremor in Hawaii, Part II. Theory and discussion, USGS professional paper 1350:1259-1280.

Crosson, R. S. and D. A. Bame (1985). A spherical source model for low-frequency volcanic earthquakes, J. Geophys. Res. 90, 10,237-10,247.

Eaton, J. P., D. H. Richter, H. L. Krivoy, (1987). Cycling of magma between the summit reservoir and Kilauea Iki lava lake during the 1959 eruption of Kilauea volcano, USGS professional paper 1350:1307-1336.

Felch, R. N. (1987). A study of the seismic structure in the Valles Caldera Region of northern New Mexico, Ph.D. thesis, Penn. State Univ., State College, 251 pp.

Ferrazzini, V., B. Chouet, M. Fenler and K. Aki (1989). Quantitative analysis of Tongperiod events recorded during hydrofracture experiments at Fenton Hill, New Mexico, in preparation. 
Ferrazzini, V. and K. Aki, Assorted seismic signals from Kilauea volcano, Hawaii, International Workshop on Volcanic Seismo gy, Capri, Italy, Oct. 1988.

Heiken, G. and F. Goff (1983). Hot dry rock geothermal energy in the Jemez Volcanic Field, New Mexico, J. Volcanol. Geotherm. Res.: 15, 223-246.

Hickman, S. H., M. D. Zoback and J. H. Healy (1988). Continuation of a deep borehole stress measurement profile near the San Andreas fault, 1 . Hydraulic fracturing stress measurements at Hi Vista Mojave Desert, California, J. Geophys. Res. 93, 15183-15195.

Hudson, J. A. (1981). Wave speeds and attenuation of elastic waves in material containing cracks, Geophys. J. R. astr. Soc., 64, 133-150.

Hudson, J. A. (1982). Overall properties of a cracked solid, Math. Proc. Camb. Phil. Soc., $88,371-384$.

Iyer, H. M. (1988). Seismological detection and delineation of Magma chambers beneath intraplate volcanic centers in western USA, in Modeling of Volcanic Processes. King, C.-Y, and R. Scarpa (Eds.), pp. 1-56, Friedr. Vieweg and Sons.

Kamo, K., T. Furuzawa, J. Akamatsu (1977). Some natures of volcanic micro-tremors at the Sakurajima volcano, Bull. Volcanol. Soc. Japan, 22:41-58.

Kolstad, C. D. and T. R. McGetchin (1978). Thermal evolution models for the Valles Caldera with reference to a hot-dry-rock geothermal experiment, J. Volc. and Geotherm. Res.: 3, 197-218.

Koyanagi, R. Y., B. Chouet, K. Aki (1987). Origin of volcanic tremors in Hawaii, Part I. Data from the Hawaiian Volcano Observatory 1969-1985, USGS professional paper 1350:1221-1258.

Minakami, T. (1974). Seismology of volcanoes in Japan. In: Physical Volcanology (eds.) Civetta, L., Gasparini, P., Luongo, G., Rajolla, A., Elsevier, Amsterdam, pp. 12-27.

Moos, D., S. H. Hickman and M. D. Zoback (1983). Sonic velocity and fracture patterns or distribution in a well drilled through the Cleveland Hill fault, Oroville, CA (abstract), EOS 64, 834.

Morse, P. M. and R. Bolt (1944). Sound waves in rooms, Rev. Modern Physics 16, p. 2, 69-150.

Morse, P. M. and K. U. Ingard (1968). Theoretical acoustics, Princeton University Press.

Morton, J. L., N. H. Sleep (1985). Seismic reflections from a Lau Basin magma chamber in geology of offshore resources of Pacific Island arcs - Tonga region, Circum Pacific Council for Energy and Mineral Resources, Earth Sciences Series 2 (eds.) Scholl D. W., Vallier, T., Am. Assoc. Pet., p. 441, Geol. Tulsa, OK.

Nakamura, K. (1977). Volcanoes as possible indicators of tectonic stress orientation principle and proposal, J. Volcanol. Geotherm. Res., 2:1-16. 
Nercessian, H., A. Hirn, A. Tarantola, (1984). Three-dimensional seismic transmission prospecting of the Mont Dore volcano, France, Geophys. J. R. astr. Soc. 17:307315.

Nielson, D. N. and J. B. Hulen (1984). Internal geology and evolution of the Redondo Dome, Valles Caldera, New Mexico, J. Geophys. Res.: 89, 8695-8711.

Nolet, Guust, Seismic tomography with applications in Global Seismology and Exploration Geophysics, Chapter 4. (E. Wielandt), On the validity of the ray approximation for interpreting delay times, D. Reidel Publishing Company.

Omer, G. C. (1950). Volcanic tremor, Bull. Seis. Soc. Am. 40:175-194.

Ramberg, H. (1970). Model studies in relation to intrusion of plutonic bodies. Mechanism of Igneous Intrusion (eds.) Newall, G., Rast, N., Gallery Press, Liverpool, pp. 261-272.

Sassa, K. (1935). Volcanic micro-tremors and eruption-earthquakes, Mem. Coll. Sci. Univ. Kyoto. Ser. A., 18:255-293.

Smith, R. L. and R. A. Bailey (1966). The Bandelier Tuff-A Study of Ash-flow Eruption Cycles from Zones Magma Chambers, Bull. Volcanol.: 29, 83-104.

Steeples, D. W. and H. M. Iyer (1976). Low-velocity zone under Long Valley as determined from teleseismic events, J. Geophys. Res. 81:849-860.

Swanson, D. A., W. Duffield, D. Jackson, D. Peterson (1979). Chronological narrative of the 969-71 Mauna Ulu eruption, Kilauea Volcano, Hawaii, USGS professional paper 1056, $55 \mathrm{p}$.

Thurber, C. H. (1984). Seismic detection of the summit magma complex of Kilauea Volcano, Hawaii, Science 223:165-167.

Thurber, C. H., K. Aki (1987). Three-dimensional seismic imaging, Ann. Rev. Earth Planet. Sci. 1987, 15:115-139.

Varadan, V. K., V. V. Varadan and Y. H. Pao (1979). Multiple scattering of elastic waves by cylinders of arbitrary cross section. I. SH waves, J. Acoust. Soc. Am., 63, 1310-1319.

Varadan, V. K., V. N. Bring and Y. Ma (1983). Coherent attenuation of acoustic waves by pair-correlated random distribution of scatterers with uniform and Gaussian size distributions, J. Acoust. Soc. Am., 73, 1941-1947.

Zoback, M. D., H. Tsukahara and S. H. Hickman (1980). Stress measurements at depth in the vicinity of the San Andreas fault: Implications for the magnitude of shear stress at depth, J. Geophys. Res., 85, 6157-6173. 


\section{BIOGRAPHICAL SKETCH KEIITI AKI}

Keiiti Aki is W. M. Keck Foundation Professor of Geological Sciences in the Department of Geological Sciences at the University of Southerm California (USC). He moved to USC in 1984 from MIT where he was R. R. Schrock Professor of Earth and Planetary Sciences in the Department of Earth and Planetary Sciences. His research activity has covered a broad area of seismology including earthquake source mechanism, attenuation, strong motion prediction, earthquake prediction, three-dimensional seismic imaging, volcanic tremor, and hydraulic fracturing. His current interests are centered around the synthesis of various seismological investigations on structures and processes in seismic and volcanic areas. Professor Aki is a member of the National Academy of Sciences, and a fellow of the American Academy of Arts and Sciences. He is also honorary foreign fellow of the European Union of Geosciences. He received the society medal from the Seismological Society of America.

He is chairman of the subcommittee on Earthquake Studies of the Advisory Committee to U. S. Geological Survey, members of the National Earthquake Prediction Evaluation Council, the California Earthquake Prediction Evaluation Council, and the Review Group for DOE's Continental Scientific Drilling Project. "Orogeny".

He coauthored books titled "Quantitative Seismology: Theory and Methods", and

The following is a selected list of his publications:

(with A. Jin) Spatial and temporal correlation between coda Q and seismicity in China. Bull. Seis. Soc. Am., 78, 741 $769,1988$.

Impact of Earthquake Seismology on the geological community since the Benioff zone, Bulletin of the Geological Soc. Am., 100, 625-629, 1988.

(with V. Ferrazzini) Slow waves trapped in a fluid-filled infinite crack; implication for volcanic tremor, J. Geophys. Res., 22, 9215-9224. 1987.

Local site effects on strong ground mution. ASCE Proceeding of the conference "Earthquake Engineering and Soil Dynamics", 1988.

Frequency-magnitude relation for small earthquakes: a clue to fmax of large earthquakes, J. Geophys. Res., 22,1349 $1335,1987$.

(with A. S. Papageorgiou), A specific barrier model for the quantitative description of inhomogeneous faulting and the prediction of strong ground motion - II. Applications of the model, Bull. Seis. Soc. Am., 73, 953-978, 1983.

Attenuation of shear waves in the lithosphere for frequencies from 0.05 to $25 \mathrm{~Hz}$. Phys. Earth. Planet. Int., 21, 50-60. 1980.

Characterization of barriers on an earhquake fault J. Geophys. Res., 84, 6140-6148, 1979.

(with A. Christoffersson and E. S. Husebye), Determination of the three-dimensional seismic structure of the lithosphere, J. Geophys. Res., 82, 277-296, 1977.

(with W. H. K. Lee), Determination of three-dimensional velocity anomalies under a seismic array using first $P$ arrival times from local earthquakes. 1. A. Homogeneous initial model, J. Geophys. Res., 81, 4381 4399, 1976.

Scattering of P waves under the Montana Lasa, J. Geophys. Res., 78, 1334-1346, 1973.

(with K. Lamer). Surface motion of a layered medium having an irregular interface due to incident plane SH waves, J. Geophys. Res., 75, 933-954, 1970.

Aralysis of the seismic coda of local earthquakes as scattered waves, J. Geophys. Res., 74, 615-631, 1969.

Seismic displacement near a faulh J. Geophys. Res., 73, 5359-5379, 1968. 
$I$
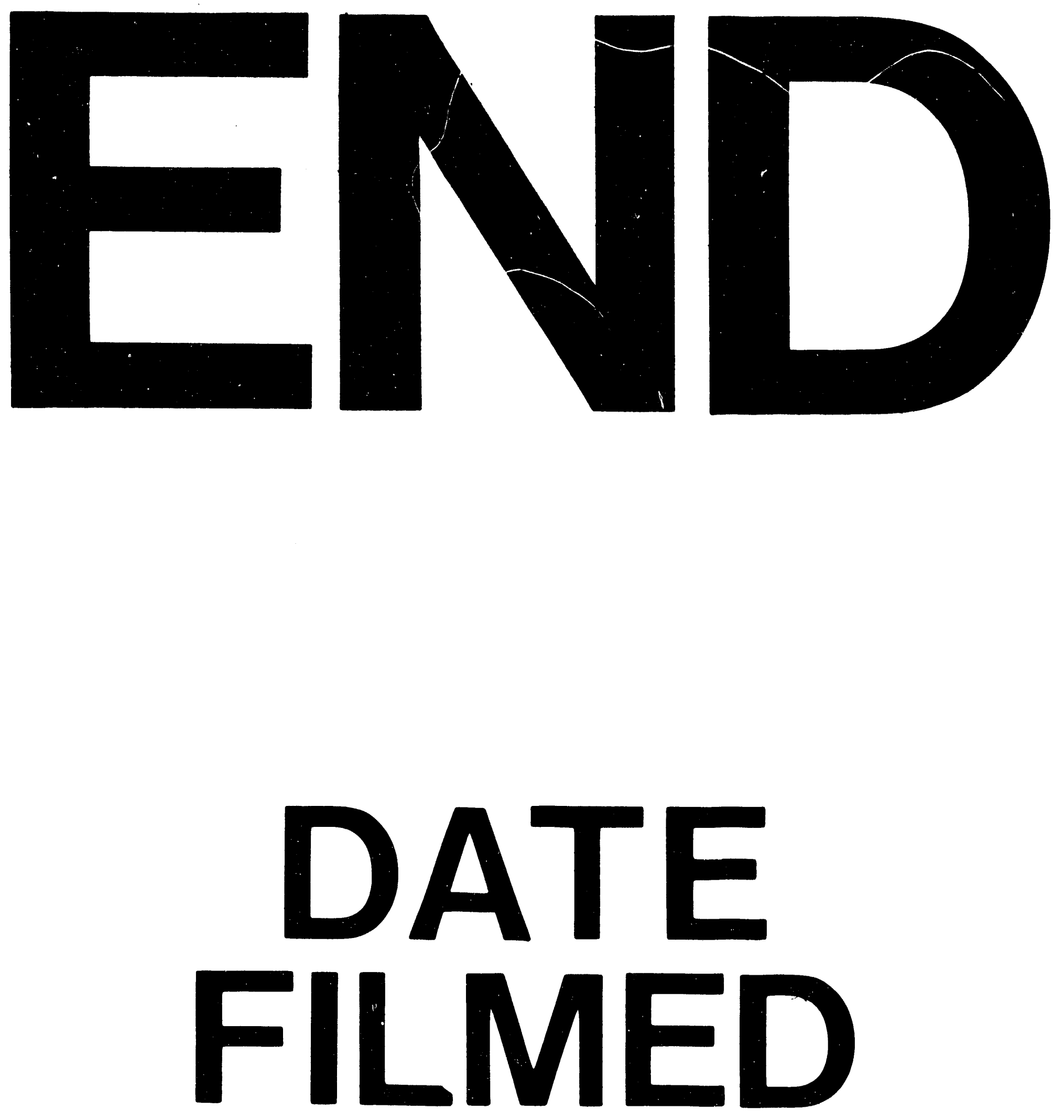

I

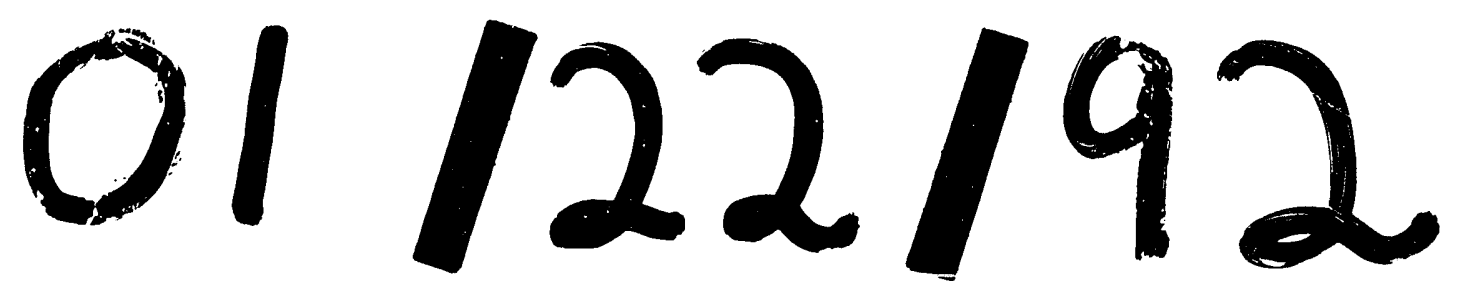


